\title{
Existence of Global Weak Solutions for Some Polymeric Flow Models
}

\author{
John W. Barrett ${ }^{\star}$, Christoph Schwab ${ }^{\dagger}$ and Endre Süli ${ }^{\ddagger}$ \\ $\star$ Department of Mathematics, Imperial College London, London SW7 2AZ, UK. \\ † Seminar for Applied Mathematics, ETH-Zentrum, HG G 58.1, CH-8092 Zürich, Switzerland. \\ $\ddagger$ University of Oxford, Computing Laboratory, Wolfson Building, Parks Road, Oxford OX1 3QD, UK.
}

August 31, 2004

\begin{abstract}
We study the existence of global-in-time weak solutions to a coupled microscopic-macroscopic beadspring model which arises from the kinetic theory of diluted solutions of polymeric liquids with noninteracting polymer chains. The model consists of the unsteady incompressible Navier-Stokes equations in a bounded domain $\Omega \subset \mathbb{R}^{d}, d=2,3$, for the velocity and the pressure of the fluid, with an extra-stress tensor as right-hand side in the momentum equation. The extra-stress tensor stems from the random movement of the polymer chains and is defined through the associated probability density function which satisfies a Fokker-Planck type degenerate parabolic equation. Upon appropriate smoothing of the convective velocity field in the Fokker-Planck equation, and in some circumstances, of the extra-stress tensor, we establish the existence of global-in-time weak solutions to this regularised bead-spring model for a general class of spring-force-potentials including in particular the widely used FENE (Finitely Extensible Nonlinear Elastic) model.
\end{abstract}

\section{Introduction}

The purpose of this paper is to explore the question of global existence of weak solutions to a set of partial differential equations which arises from the kinetic theory of the flow of a diluted solution of polymeric liquid in a domain $\Omega \subset \mathbb{R}^{d}, d=2,3$. The simplest model of this kind to account for noninteracting polymer chains is the so-called dumbbell model (cf. [3]); a dumbbell consists of two beads connected by an elastic spring. Following [4], at time $t$ the dumbbell is characterised by the position of its centre of mass $\underset{\sim}{X}(t)$ and its elongation vector $Q(t)$ (see Figure 1). When a dumbbell is placed into a given velocity field $\underset{\sim}{u}(\underset{\sim}{x}, t)$, three forces act on each bead: the first force is the drag force proportional to the difference between the bead velocity and the velocity of the surrounding fluid particles; the second force is the elastic force $\underset{\sim}{F}$ due to the spring stiffness; the third force is due to thermal agitation and is modelled by Brownian motion.

On rescaling the elongation vector, Newton's equations of motion for the beads give rise to 


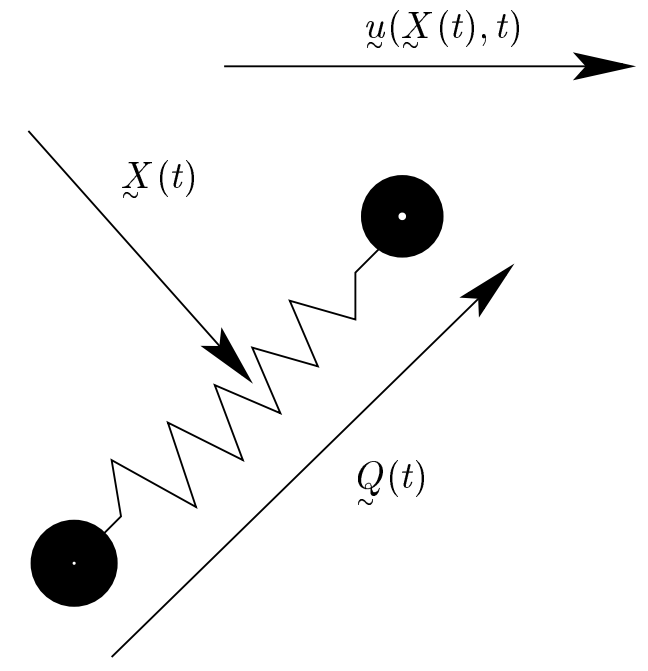

Figure 1: Noninteracting polymer chains, immersed into an incompressible flow with velocity $\underset{\sim}{u}$, are modelled by using dumbbells, each dumbbell representing a polymer chain. A dumbbell is a pair of beads connected with an elastic spring. At time $t \geq 0$, the dumbbell is characterised by the position $\underset{\sim}{X}(t)$ of its centre of mass and its elongation vector $\underset{\sim}{Q(t)}$.

the following set of stochastic differential equations:

$$
\begin{aligned}
\mathrm{d} X & =\underset{\sim}{u}(\underset{\sim}{X}(t), t) \mathrm{d} t, \\
\mathrm{~d} Q & \left.\left.=\left(\underset{\sim}{\nabla}{\underset{\sim}{\sim}}_{\sim} \underset{\sim}{(X}(t), t\right) \underset{\sim}{Q}(t)-\frac{1}{2 \lambda} \underset{\sim}{F} \underset{\sim}{Q}(t)\right)\right) \mathrm{d} t-\frac{1}{\sqrt{\lambda}} \mathrm{d} \underset{\sim}{W}(t) ;
\end{aligned}
$$

where $\underset{\sim}{W}$ is a vector of independent scalar Wiener processes, $\underset{\sim}{F}(Q)$ denotes the elastic force acting on the chain due to elongation, the positive parameter $\lambda=\xi /(4 H)$ characterises the elastic property of the fluid, with $\xi$ denoting the drag coefficient and $H$ the spring stiffness. For details, we refer, for example, to $[3,6,10,17]$.

The present paper is concerned with the well-posedness of the deterministic restatement of this problem. For this purpose, let $\psi(\underset{\sim}{x}, \underset{\sim}{q}, t)$ denote the probability density function corresponding to the vector-valued stochastic process $\underset{\sim}{X}(t), Q(t))$; thus, $\psi(\underset{\sim}{x}, \underset{\sim}{q}, t)$ represents the probability, at time $t$, of finding the centre of mass of a dumbbell at a position 'between' $\underset{\sim}{x}$ and $\underset{\sim}{x}+\mathrm{d} \underset{\sim}{x}$ and having elongation 'between' $\underset{\sim}{q}$ and $\underset{\sim}{q}+\mathrm{d} q$.

Now, let us suppose that the elastic force $\underset{\sim}{F}: D \subseteq \mathbb{R}^{d} \rightarrow \mathbb{R}^{d}, d=2,3$, of the spring is defined through a (sufficiently smooth) potential $U: \mathbb{R}_{\geq 0} \rightarrow \mathbb{R}$ via

$$
\underset{\sim}{F}(\underset{\sim}{q})=U^{\prime}\left(\frac{1}{2}|\underset{\sim}{q}|^{2}\right) \underset{\sim}{q} .
$$

Then, the evolution of the probability density function $\psi(\underset{\sim}{x}, q, t)$ of the stochastic process $(\underset{\sim}{X}(t), \underset{\sim}{Q}(t))$ defined by $(1.1 \mathrm{a}, \mathrm{b})$ satisfies the Fokker-Planck equation $(2.4 \mathrm{a})$ below for $(\underset{\sim}{x}, \underset{\sim}{q}, t) \in$ $\Omega \times D \times \mathbb{R}_{>0}$, subject to appropriate initial and boundary conditions; cf. (2.4b,c). Due to the fact that, unlike (1.1b), the differential equation (1.1a) does not involve random effects, the Fokker-Planck equation for the associated probability density function is a degenerate parabolic equation for $\psi(\underset{\sim}{x}, \underset{\sim}{q}, t)$, with no diffusion in the $\underset{\sim}{x}$-direction. The velocity field $\underset{\sim}{u}$ appearing in 
(2.4a) is, in turn, found by solving the incompressible Navier-Stokes equations (2.1a,b) below, subject to the initial and boundary conditions $(2.1 \mathrm{c}, \mathrm{d})$. The right-hand side of the momentum equation (2.1a) models the non-Newtonian effects through the presence of the extra-stress tensor $\underset{\approx}{\tau}$ which depends on the probability density function $\psi$ (see, (2.2), (2.3)). Our aim here is to explore the existence of global-in-time solutions of this coupled 'microscopic-macroscopic' model.

An early effort to show the existence and uniqueness of local-in-time solutions to a family of bead-spring type polymeric flow models is due to Renardy [18]. While the class of potentials $\underset{\sim}{F}(q)$ considered in [18] (cf. hypotheses (F) and (F') on pp.314-315) does include the case of Hookean dumbbells, with $\underset{\sim}{F}(\underset{\sim}{q})=\underset{\sim}{q}$, it excludes the practically relevant case of the FENE (Finitely Extensible Nonlinear Elastic) model where

$$
\underset{\sim}{F}(\underset{\sim}{q})=\left(1-\frac{|\underset{q}{\mid q}|^{2}}{b}\right)^{-1} \underset{\sim}{q}, \quad|\underset{\sim}{q}|<b, \quad b>0 .
$$

In a recent paper Jourdain, Lelièvre and le Bris [11] studied the existence of solutions to the FENE model in the case of a simple Couette flow; by using tools from the theory of stochastic differential equations, they established the existence of a unique local-in-time solution to the FENE model in two space dimensions $(d=2)$ when the velocity field $\underset{\sim}{u}$ is unidirectional and of the particular form $\underset{\sim}{u}\left(x_{1}, x_{2}\right)=\left(u_{1}\left(x_{2}\right), 0\right)^{\top}$. The notion of solution for which existence is proved in [11] is mixed deterministic-stochastic in the sense that it is deterministic in the 'macroscopic' variable $x$, but stochastic in the 'microscopic' variable $q$. In contrast, our notion of solution (cf. Section 3 below) is deterministic both macroscopically and microscopically, since the microscales are modelled here by the probability density function $\psi(\underset{\sim}{x}, \underset{\sim}{q}, t)$. The choice between these different notions of solution has far-reaching repercussions concerning computational simulation: mixed deterministic-stochastic notions of solution necessitate the use of Monte Carlo-type algorithms for the numerical approximation of polymer configurations, as proposed, e.g., in [17] and references therein as well as in [10]; whereas weak solutions in the sense considered in the present paper can be approximated by entirely deterministic (e.g. Galerkin-type) schemes, as has been done, for example, in [16].

In the case of Hookean dumbbells, the coupled microscopic-macroscopic model described above yields, formally, on taking the second moment of $q \mapsto \psi(\underset{\sim}{q}, \underset{\sim}{x}, t)$, the fully macroscopic, Oldroyd-B model of visco-elastic flow (cf. Section 2.2). In [15], Lions and Masmoudi show the existence of global-in-time weak solutions to the Oldroyd-B model in a simplified case which, in our coupled microscopic-macroscopic setup, directly corresponds to assuming that the drag term in (2.4a) is corotational: that is, the tensor $\underset{\sim}{\sigma(u)}$ is skew-symmetric in the sense that $\underset{\widetilde{c}}{\sigma(u)}=-[\underset{\sim}{\sigma}(u)]^{\top}$. The argument in [15] is based on exploiting the propagation in time of the compactness of the solution. It is not known if an identical global existence result for the Oldroyd-B model also holds in the absence of the crucial assumption that the drag term is corotational.

The present paper is concerned with the proof of existence of global-in-time weak solutions to the coupled microscopic-macroscopic model $(2.1 \mathrm{a}-\mathrm{d}),(2.4 \mathrm{a}-\mathrm{c})$. Our hypotheses on the potential $U$ admit a fairly large class of models, including the Hookean dumbbell model as well as general FENE-type models. Unlike [15], we do not need to assume that the flow is corotational in the FENE case. However, the level of generality pursued here comes at a price: in order to complete our existence proofs, the velocity field appearing in the drift-term of the Fokker-Planck 
equation (2.4a) had to be suitably mollified in the case of corotational microscopic-macroscopic models, and in the case of general, noncorotational models, the extra-stress tensor $\underset{\sim}{\tau}$ on the right-hand side of (2.1a) had to be mollified also. A possible physical justification for the proposed modification of the original mathematical model, through smoothing the velocity field in particular terms in the model, is that many flows of diluted solutions of polymers tend to be slow, with a smoothly varying velocity field.

The mollification of the velocity field considered here is stimulated by the Leray- $\alpha$ model of the incompressible Navier-Stokes equations (the viscous Camassa-Holm equations), proposed by Foias, Holm and Titi [8], in a bounded open set $\Omega \subset \mathbb{R}^{d}$ with boundary $\partial \Omega$ :

Find $\underset{\sim}{u}:(\underset{\sim}{x}, t) \in \mathbb{R}^{d+1} \mapsto \underset{\sim}{u}(\underset{\sim}{x}, t) \in \mathbb{R}^{d}, d=2,3$, and $p:(\underset{\sim}{x}, t) \in \mathbb{R}^{d+1} \mapsto p(\underset{\sim}{x}, t) \in \mathbb{R}$ such that

$$
\begin{aligned}
& \left.\frac{\partial u}{\partial t}+\underset{\sim}{v} \cdot \underset{\sim}{\nabla_{x}}\right) \underset{\sim}{u}-\nu \Delta_{x} \underset{\sim}{u}+\underset{\sim}{\nabla_{x}} p=\underset{\sim}{f} \quad \text { in } \Omega \times(0, T], \\
& \underset{\sim}{\nabla} \cdot \underset{\sim}{u}=0 \quad \text { in } \Omega \times(0, T], \\
& \underset{\sim}{u}=0 \quad \text { on } \partial \Omega \times(0, T], \\
& \underset{\sim}{u}(\underset{\sim}{x}, 0)=\underset{\sim}{u_{0}}(\underset{\sim}{x}) \quad \forall \underset{\sim}{\forall x} \in \Omega
\end{aligned}
$$

where $\underset{\sim}{u}$ is the velocity field, $p$ is the pressure of the fluid, $\nu \in \mathbb{R}_{>0}$ is the viscosity, $\underset{\sim}{f}$ is a given body force, and $\underset{\sim}{v}(\cdot, t)$ is a smoothing of $\underset{\sim}{u}(\cdot, t)$ defined as the solution of the Helmholtz problem

$$
\begin{array}{rlrl}
\underset{\sim}{v}-\alpha \Delta_{x} \underset{\sim}{v} & =\underset{\sim}{u} & \text { in } \Omega, \\
\underset{\sim}{v}=\underset{\sim}{0} & \text { on } \partial \Omega ;
\end{array}
$$

where $\alpha>0$ is a regularisation parameter.

With $\underset{\sim}{v}$ thus defined one cannot deduce from $(1.4 \mathrm{a}, \mathrm{b})$ that $\underset{\sim}{\underset{\sim}{\nabla}} \cdot \underset{\sim}{v}=0$ on $\Omega$. As we would like $\underset{\sim}{v}$ to resemble $\underset{\sim}{u}$ both in terms of its incompressibility and the boundary conditions it satisfies, instead of $(1.4 \mathrm{a}, \mathrm{b})$ we shall seek the smoothing $\underset{\sim}{v}$ of $\underset{\sim}{u}$ from the following Helmholtz-Stokes problem

$$
\begin{aligned}
& \underset{\sim}{v}-\alpha \Delta_{x} \underset{\sim}{v}+\underset{\sim}{\nabla_{x}} \pi=\underset{\sim}{u} \quad \text { in } \Omega \\
& \underset{\sim}{\nabla} \cdot \underset{\sim}{v}=0 \quad \text { in } \Omega, \\
& v=0 \quad \text { on } \partial \Omega
\end{aligned}
$$

where $\pi$ is a pressure-like auxiliary variable. Since, strictly speaking, $\pi$ does not have a physical meaning, it is best thought of as a Lagrange multiplier whose role is to enforce incompressibility.

We remark that smoothing, albeit of a somewhat different kind, is also conceivable on physical grounds, since equations $(1.1 \mathrm{a}, \mathrm{b})$ could be modified to

$$
\begin{aligned}
& \mathrm{d} \underset{\sim}{X}=\underset{\sim}{u} \underset{\sim}{X}(t), t) \mathrm{d} t+\varepsilon \underset{\sim}{\operatorname{d}} \bar{W}(t), \\
& \left.\left.\mathrm{d} Q=\left(\underset{\sim}{\nabla_{X}} \underset{\sim}{u} \underset{\sim}{X}(t), t\right) \underset{\sim}{Q} Q(t)-\frac{1}{2 \lambda} \underset{\sim}{F} \underset{\sim}{Q}(t)\right)\right) \mathrm{d} t-\frac{1}{\sqrt{\lambda}} \mathrm{d} W(t) ;
\end{aligned}
$$

where $\varepsilon>0$ is a small parameter and $\underset{\sim}{W}(t)$ is an independent vector of Wiener processes modelling, for example, thermal vibrations of the (center of gravity) of the dumbbell. A closely related smoothing on the macroscopic level is considered in the work of El-Kareh and Leal [7]. 
The paper is structured as follows. In Section 2, we formulate the class of models considered. As an illustration, we show how the Hookean dumbbell model and the FENE model fit into the general setting. In Section 3 we introduce a family of weighted Sobolev spaces which represent the natural setting for the problem; we also establish crucial density and trace results. In order to motivate the energy estimates which, ultimately, via weak compactness, lead to the existence of global weak solutions, we establish formal energy estimates and introduce our smoothing operator. We then use these to rigorously define our weak formulation of the problem. In Section 4 we embark on the proof of existence of global weak solutions, starting with the analysis of the simpler, corotational case for both Hookean and FENE-type models. We conclude with the proof of existence of global weak solutions for the physically more realistic FENE-type models in the general case, without assuming that the drag term is corotational.

\section{Polymer models}

We term polymer models under consideration here microscopic-macroscopic type models, since the continuum mechanical macroscopic equations of incompressible fluid flow are coupled to a microscopic model: the Fokker-Planck equation describing the statistical properties of particles in the continuum. We first present these equations and collect assumptions on the parameters in the model.

\subsection{Microscopic-macroscopic polymer models}

Let $\Omega \subset \mathbb{R}^{d}$ be a bounded open set with a Lipschitz-continuous boundary $\partial \Omega$, and suppose that the set $D \subseteq \mathbb{R}^{d}, d=2$ or 3 , of admissible elongation vectors $q$ in (1.1b) is an open set which may be bounded or unbounded. For the sake of simplicity of presentation, we shall suppose that $D$ is either a bounded open ball in $\mathbb{R}^{d}$, or $D=\mathbb{R}^{d}$; these two cases cover all practically relevant scenarios involving the microscopic-macroscopic models discussed here. Our arguments in the case when the configuration domain $D$ is a bounded open ball can be extended, with only minimal changes, to situations when $D$ is any bounded open domain in $\mathbb{R}^{d}$ with smooth boundary (e.g. an ellipse, to account for anisotropy in the molecule's configuration).

We consider the following initial-boundary-value problem.

(P) Find $\underset{\sim}{u}:(\underset{\sim}{x}, t) \in \mathbb{R}^{d+1} \mapsto \underset{\sim}{u}(\underset{\sim}{x}, t) \in \mathbb{R}^{d}$ and $p:(\underset{\sim}{x}, t) \in \mathbb{R}^{d+1} \mapsto p(\underset{\sim}{x}, t) \in \mathbb{R}$ such that

$$
\begin{aligned}
& \frac{\partial u}{\partial t}+\left(\underset{\sim}{u} \cdot \underset{\sim}{\nabla_{x}}\right) \underset{\sim}{u}-\nu \Delta_{x} \underset{\sim}{u}+\underset{\sim}{\nabla_{x}} p=\underset{\sim}{\nabla_{x}} \cdot \underset{\sim}{\tau}(\psi) \quad \text { in } \Omega \times(0, T], \\
& \underset{\sim}{\nabla_{x}} \cdot \underset{\sim}{u}=0 \quad \text { in } \Omega \times(0, T], \\
& u=0 \quad \text { on } \partial \Omega \times(0, T], \\
& \underset{\sim}{u}(\underset{\sim}{x}, 0)=\underset{\sim}{u_{0}}(\underset{\sim}{x}) \quad \forall \underset{\sim}{\forall} \in \Omega
\end{aligned}
$$

where $\nu \in \mathbb{R}_{>0}$ is the viscosity and $\underset{\sim}{\tau}(\psi):(\underset{\sim}{x}, t) \in \mathbb{R}^{d+1} \mapsto \underset{\sim}{\tau}(\psi)(\underset{\sim}{x}, t) \in \mathbb{R}^{d \times d}$ is the symmetric extra-stress tensor, dependent on a probability density function $\psi:(\underset{\sim}{x}, \underset{\sim}{q}, t) \in \mathbb{R}^{2 d+1} \mapsto$ 
$\psi(\underset{\sim}{x}, \underset{\sim}{q}, t) \in \mathbb{R}$, defined as

$$
\underset{\approx}{\tau}(\psi)=k \mu \underset{\approx}{C}(\psi)-\rho(\psi) \underset{\approx}{I}) .
$$

Here $k, \mu \in \mathbb{R}_{>0}$ are, respectively, the Boltzmann constant and the absolute temperature, $\underset{\approx}{I}$ is the unit $d \times d$ tensor, and

$$
\underset{\approx}{C}(\psi) \underset{\sim}{x}, t)=\int_{D} \psi(\underset{\sim}{x}, \underset{\sim}{q}, t) U^{\prime}\left(\frac{1}{2}|q|_{\sim}^{2}\right) \underset{\sim}{q} \underset{\sim}{q} \mathrm{~d} \underset{\sim}{q} \quad \text { and } \quad \rho(\psi) \underset{\sim}{(x, t)}=\int_{D} \psi \underset{\sim}{\psi(\underset{\sim}{x}, \underset{\sim}{q}, t)} \mathrm{d} \underset{\sim}{q} .
$$

In addition, the real-valued, continuous, nonnegative and strictly monotonic increasing function $U$, defined on a relatively open subset of $[0, \infty)$, is an elastic potential which gives the elastic force $\underset{\sim}{F}: D \rightarrow \mathbb{R}^{d}$ on the springs via (1.2).

The probability density $\psi(\underset{\sim}{x}, \underset{\sim}{q}, t)$ represents the probability at time $t$ of finding a dumbbell located 'between' $\underset{\sim}{x}$ and $\underset{\sim}{x}+\mathrm{d} \underset{\sim}{x}$ having elongation 'between' $\underset{\sim}{q}$ and $\underset{\sim}{q}+\mathrm{d} q$. Hence $\rho(\underset{\sim}{x}, t)$ is the density of the polymer chains located at $\underset{\sim}{x}$ at time $t$. It follows from $(1.1 \mathrm{a}, \mathrm{b})$ that $\psi$ satisfies the Fokker-Planck equation, together with suitable boundary and initial conditions:

$$
\begin{aligned}
& \left.\left.\frac{\partial \psi}{\partial t}+\underset{\sim}{u} \cdot \underset{\sim}{\nabla} x\right) \psi+\underset{\sim}{\nabla} q \cdot \underset{\sim}{\sigma} \underset{\sim}{u} \underset{\sim}{q} \psi\right)=\frac{1}{2 \lambda} \underset{\sim}{\nabla} q \cdot\left(\underset{\sim}{\nabla_{q}} \psi+U^{\prime} \underset{\sim}{q} \psi\right) \quad \text { in } \Omega \times D \times(0, T], \\
& \psi=0 \quad \text { on } \Omega \times \partial D \times(0, T], \\
& \psi(\underset{\sim}{x}, \underset{\sim}{q}, 0)=\psi_{0}(\underset{\sim}{x}, \underset{\sim}{q}) \geq 0 \quad \forall \underset{\sim}{x, q} \underset{\sim}{q} \in \mathbb{R} \in D .
\end{aligned}
$$

When $D=\mathbb{R}^{d}$, the boundary condition (2.4b) on $\partial D$, the boundary of $D$, is replaced by a decay condition at infinity which demands that $|\psi|$ converges to 0 sufficiently fast as $|\underset{\sim}{q}|$ tends to $\infty$; we shall be more specific about this in Lemma $3.2(\mathrm{~b})$.

In (2.4a) the parameter $\lambda \in \mathbb{R}_{>0}$ characterises the elastic properties of the fluid, and $\underset{\approx}{\sigma}(\underset{\sim}{x}, t) \in$

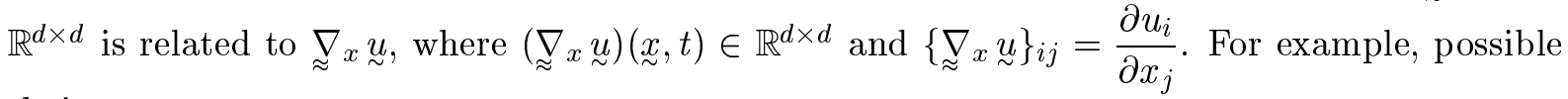
choices are

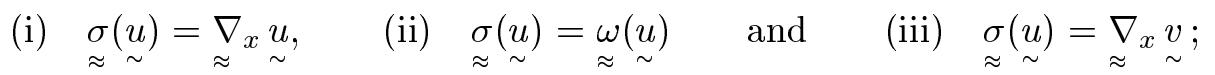

where

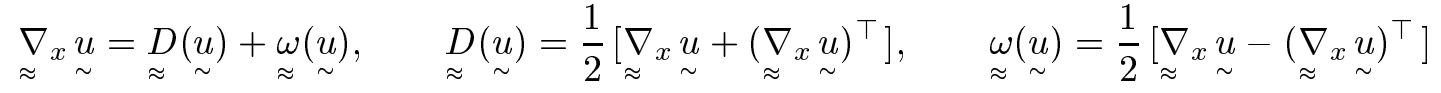

and $\underset{\sim}{v}$ is a 'smoothed' version of $\underset{\sim}{u}$. The precise form of the smoothing operator which maps $\underset{\sim}{u}$ into $\underset{\sim}{v}$ will be given in Section 3.3.

On introducing the (normalised) Maxwellian

$$
M(\underset{\sim}{q})=\frac{\mathrm{e}^{-U\left(\frac{1}{2}|q|^{2}\right)}}{\int_{D} \mathrm{e}^{-U} \underset{\sim}{\mathrm{d} q}},
$$

we have

$$
M \underset{\sim}{\underset{\sim}{\nabla}} M^{-1}=-M^{-1} \underset{\sim}{\nabla_{q}} M=U^{\prime} \underset{\sim}{q}
$$


In addition, the following identities hold:

$$
\underset{\sim}{\nabla_{q}} U=U^{\prime} \underset{\sim}{q}, \quad \underset{\sim}{\nabla_{q}} U^{\prime}=U^{\prime \prime} \underset{\sim}{q} \quad \text { and } \quad \Delta_{q} U=U^{\prime \prime}|\underset{\sim}{\mid q}|^{2}+U^{\prime} d .
$$

Thus, the Fokker-Planck equation (2.4a) can be rewritten as

$$
\left.\frac{\partial \psi}{\partial t}+\underset{\sim}{(u} \cdot \underset{\sim}{\nabla_{x}}\right) \psi+\underset{\sim}{\nabla_{q}} \cdot \underset{\sim}{\sigma(u)} \underset{\sim}{q} \psi(\underset{\sim}{q} \psi)=\frac{1}{2 \lambda} \underset{\sim}{\nabla_{q}} \cdot\left(M \underset{\sim}{\nabla_{q}}\left(\frac{\psi}{M}\right)\right) \quad \text { in } \Omega \times D \times(0, T] .
$$

\subsection{Two examples}

1. FENE-type models. A widely used model is the FENE (Finitely Extensible Nonlinear Elastic) model, where

$$
\left.D=B \underset{\sim}{0}, b^{\frac{1}{2}}\right) \text { and } U(s)=-\frac{b}{2} \ln \left(1-\frac{2 s}{b}\right) \text {, and hence } e^{-U\left(\frac{1}{2}|q|^{2}\right)}=\left(1-\frac{|q|^{2}}{b}\right)^{\frac{b}{2}} \text {. }
$$

Here $B(\underset{\sim}{0}, s)$ is the bounded open ball of radius $s>0$ in $\mathbb{R}^{d}$ centred at the origin, and $b>0$ is an input parameter. Hence the elongation $|\underset{\sim}{\mid}|$ cannot exceed $b^{\frac{1}{2}}$.

2. Hookean dumbbells. Letting $b \rightarrow \infty$ in (2.10) leads to the so-called Hookean dumbbell model where

$$
D=\mathbb{R}^{d} \quad \text { and } \quad U(s)=s, \quad \text { and therefore } \quad \mathrm{e}^{-U\left(\frac{1}{2}|q|^{2}\right)} \underset{\sim}{\stackrel{-\frac{1}{2}|q|^{2}}{\sim} .}
$$

This particular kinetic model, with $\underset{\sim}{\sigma}(\underset{\sim}{u})=\underset{\approx}{\nabla} \underset{\sim}{u}$, corresponds formally to an Oldroyd-B model, or with $\underset{\sim}{\sigma}(\underset{\sim}{u})=\underset{\sim}{\omega}(\underset{\sim}{u})$ to a corotational Oldroyd-B model. Indeed, on multiplying (2.4a) by $\underset{\sim}{q} \underset{\sim}{q}$, integrating over $D$, performing integration by parts (assuming that $\psi$ and $\left|\nabla_{q} \psi\right|$ decay to zero sufficiently fast with $|\underset{\sim}{q}| \rightarrow \infty)$, and noting for any $\underset{\sim}{r} \in \mathbb{R}^{d}$ that

$$
(\underset{\sim}{r} \underset{\sim}{\nabla} q) \underset{\sim}{q} \underset{\sim}{q}=\underset{\sim}{r} \underset{\sim}{q} q^{\top}+\underset{\sim}{q} r^{\top} \quad \text { and } \quad \Delta_{q}\left(\underset{\sim}{q} q^{\top}\right)=2 \underset{\sim}{I}
$$

yields

$$
\lambda \frac{\delta C}{\delta t}+\underset{\approx}{\approx}=\rho I \quad \text { in } \Omega \times(0, T]
$$

where

$$
\left.\left.\left.\frac{\delta C}{\delta t}=\frac{\partial C}{\partial t}+\underset{\sim}{(u} \cdot \underset{\sim}{\nabla} x\right) \underset{\approx}{C}-\underset{\approx}{\sigma} \underset{\sim}{\sigma} \underset{\approx}{C}+\underset{\approx}{C} \underset{\approx}{\sigma}(u)\right]^{\top}\right]
$$

is the upper-convected time derivative. Combining (2.13) and (2.2) and observing that the density $\rho(\underset{\sim}{x}, t)$ satisfies

$$
\left.\frac{\partial \rho}{\partial t}+\underset{\sim}{u} \cdot \underset{\sim}{\nabla_{x}}\right) \rho=0 \quad \text { in } \Omega \times(0, T]
$$

implies that the extra-stress $\underset{\approx}{\tau}(\underset{\sim}{x}, t)$ satisfies

$$
\left.\lambda \underset{\approx \tau}{\delta \tau}+\underset{\approx}{\delta t}=k \mu \lambda \rho[\underset{\approx}{\sigma} \underset{\sim}{\sigma})+\underset{\approx}{\sigma} \underset{\sim}{\sigma}(u)]^{\top}\right] \quad \text { in } \Omega \times(0, T]
$$

which is the Oldroyd-B constitutive equation if $\underset{\sim}{\sigma(u)})=\underset{\sim}{\underset{\sim}{\nabla}} \underset{\sim}{u}$ or the corotational Oldroyd-B constitutive equation if $\underset{\sim}{\sigma} \underset{\sim}{\sim})=\underset{\sim}{\omega}(\underset{\sim}{\sim})$; in the latter case, the right-hand side of (2.16) is identically equal to 0. 


\subsection{General structural assumptions on the potential}

Suppose that $D$ is a bounded open ball in $\mathbb{R}^{d}$ or $D=\mathbb{R}^{d}$. We assume that $\underset{\sim}{q} \mapsto U\left(\frac{1}{2}|\underset{\sim}{q}|^{2}\right) \in C^{\infty}(D)$ with $\underset{\sim}{q} \mapsto U\left(\frac{1}{2}|q|^{2}\right)$ nonnegative and $\underset{\sim}{q} \mapsto U^{\prime}\left(\frac{1}{2}|\underset{\sim}{q}|^{2}\right)$ positive on $D$, and that there exist constants $c_{i}>\tilde{0}, i=1,2$, such that

$$
\left(U^{\prime}\right)^{2}-U^{\prime \prime} \geq c_{1} \quad \underset{\sim}{\forall} \in D \quad \text { and } \quad\left(U^{\prime}\right)^{2}-U^{\prime \prime} \geq\left. 2 c_{2} U^{\prime} \quad \underset{\sim}{\forall} \underset{\sim}{\mid} \underset{\sim}{\mid q}\right|^{2} \geq \frac{d}{c_{2}},
$$

where $B\left(\underset{\sim}{0},\left(\frac{d}{c_{2}}\right)^{\frac{1}{2}}\right) \subset \subset D$.

The above assumptions hold for the Hookean case, (2.11), with $c_{1}=2 c_{2}=1$; and the FENE case, (2.10), on assuming that $b>2$, with $c_{1}=\frac{b-2}{b}$ and $c_{2}=\frac{b+2 d-2}{2 b}$.

We shall also suppose that there exist positive constants $c_{i}, i=3, \ldots, 7$, and $\kappa>0$, such that the Maxwellian $M$ and the associated elastic potential $U$ satisfy

$$
\begin{array}{cc}
c_{3}[\operatorname{dist}(q, \partial D)]^{\kappa} \leq M(\underset{\sim}{q}) \leq c_{4}\left[\underset{\sim}{\operatorname{dist}(q, \partial D)]^{\kappa}} \quad\right. & \underset{\sim}{\forall} \in D, \\
c_{5} \leq[\operatorname{dist}(\underset{\sim}{q}, \partial D)] U^{\prime}(\underbrace{1}_{\sim}|q|^{2}) \leq c_{6}, \quad \sim \underset{\sim}{[\operatorname{dist}(\underset{\sim}{q}, \partial D)]^{2}}\left|U^{\prime \prime}\left(\frac{1}{2}|q|^{2}\right)\right| \leq c_{7} & \underset{\sim}{\forall} \in D ;
\end{array}
$$

when $D=\mathbb{R}^{d}$, then $[\operatorname{dist}(\underset{\sim}{q}, \partial D)]^{\kappa}$ in $(2.18 \mathrm{a})$ is replaced by $\exp \left(-|\underset{\sim}{q}|^{2}\right)$, and $[\operatorname{dist}(\underset{\sim}{q}, \partial D)]$ and $[\operatorname{dist}(\underset{\sim}{q}, \partial D)]^{2}$ in $(2.18 \mathrm{~b})$ are omitted.

It is an easy matter to show that the Maxwellian $M$ and the elastic potential $U$ of the FENE model and of the Hookean dumbbell model satisfy conditions $(2.18 \mathrm{a}, \mathrm{b})$, - with $D=B\left(\underset{\sim}{0}, b^{\frac{1}{2}}\right)$ and $\kappa=b / 2$ in the case of the FENE model; and $D=\mathbb{R}^{d}$ for the Hookean dumbbell model.

We shall also require that

$$
\int_{D}\left[1+\left(1+|\underset{\sim}{q}|^{2}\right)\left((U)^{2}+|\underset{\sim}{q}|^{2}\left(U^{\prime}\right)^{2}\right)\right] M \underset{\sim}{q}<\infty
$$

For the Hookean model (2.11) and the FENE model (2.10), with $b>2,(2.19)$ is easily shown to hold. For example, we have that

$$
\mathcal{M}:=\left.\int_{D} M\left(U^{\prime}\right)^{2} \underset{\sim}{\mid q}\right|^{4} \underset{\sim}{\mathrm{d}} \underset{\sim}{q}<\infty
$$

for both models. In the Hookean case, (2.20) follows since

$$
\int_{0}^{\infty} \mathrm{e}^{-s} s^{\frac{d+2}{2}} \mathrm{~d} s<\infty
$$

while in the FENE case, (2.20) follows since

$$
\int_{0}^{b}\left(1-\frac{s}{b}\right)^{\frac{b-4}{2}} s^{\frac{d+2}{2}} \mathrm{~d} s<\infty \quad \text { if } b>2 .
$$

More generally, it follows from $(2.18 \mathrm{a}, \mathrm{b})$, on noting that $U\left(\frac{1}{2}|\underset{\sim}{q}|^{2}\right)=-\log _{\mathrm{e}} M(\underset{\sim}{q})+$ Const., that (2.19) holds provided that either: (i) $\kappa>1$ when $D$ is a bounded open ball in $\mathbb{R}^{d}$; or (ii) when $D=\mathbb{R}^{d}$. 


\section{Weak solutions}

To define an appropriate notion of weak solution, we first introduce some function spaces, then derive formal energy identities and estimates satisfied by the weak solution. These estimates will, later on, form the basis of our proof of existence. In our notion of weak solution we will also rely on smoothing operators to compensate for the lack of regularization in the hyperbolic part of the Fokker-Planck equation.

\subsection{Function spaces and embedding results}

We employ the usual function spaces for viscous, incompressible flow (e.g. [9]):

$$
\begin{aligned}
\underset{\sim}{H} & :=\left\{\underset{\sim}{w} \in \underset{\sim}{L^{2}}(\Omega): \underset{\sim}{\nabla_{x}} \cdot \underset{\sim}{w}=0\right\}, \\
\underset{\sim}{V} & :=\left\{\underset{\sim}{\underset{\sim}{w}} \in \underset{\sim}{H_{0}^{1}}(\Omega): \underset{\sim}{\nabla_{x}} \cdot \underset{\sim}{w}=0\right\}, \\
\text { and } \quad L_{0}^{2}(\Omega) & :=\left\{r \in L^{2}(\Omega): \int_{\Omega} r \underset{\sim}{\mathrm{d} x}=0\right\},
\end{aligned}
$$

where the divergence operator $\nabla_{x}$. is to be understood in the sense of vector-valued distributions on $\Omega$. In addition, we introduce the following function spaces for $\psi$ :

$$
\begin{aligned}
& K:=\left\{\varphi \in L_{\mathrm{loc}}^{1}(\Omega \times D): \int_{\Omega \times D}\left[\frac{|\varphi|^{2}}{M}+\left.M||_{\sim} \nabla_{q}\left(\frac{\varphi}{M}\right)\right|^{2}\right] \underset{\sim}{\mathrm{d} q} \underset{\sim}{\mathrm{d}} x<\infty\right\}, \\
& K_{q}:=\left\{\varphi \in K: \int_{\Omega \times D} \underset{\sim}{|q|^{2}} \frac{|\varphi|^{2}}{M} \underset{\sim}{\mathrm{d}} \underset{\sim}{\mathrm{d} x}<\infty\right\}, \\
& \left.K^{+}:=\{\varphi \in K: \varphi \underset{\sim}{x}, q) \geq 0 \text { for a.e. } \underset{\sim}{(x, q)} \in \Omega \times D\right\} \text {, }
\end{aligned}
$$

Clearly, if $D$ is bounded then $K_{q}=K$ and $K_{q}^{+}=K^{+}$. We remark, in particular, that due to the structural hypotheses on $U$ (specifically, (2.19)), both $M$ and $M U$ belong to $K_{q}^{+}$. It is helpful to note for future purposes that, more generally, a distribution $\varphi$ belongs to $K$ if, and only if, $\chi=\frac{\varphi}{M}$ has finite norm

$$
\|\chi\|_{H^{0,1}(\Omega \times D ; M)}:=\left\{\int_{\Omega \times D} M\left[|\chi|^{2}+\left|\nabla_{q} \chi\right|^{2}\right] \underset{\sim}{\mathrm{d} q} \mathrm{~d} \underset{\sim}{x}\right\}^{\frac{1}{2}} .
$$

As the corresponding weighted Sobolev space

$$
H^{0,1}(\Omega \times D ; M):=\left\{\chi \in L_{\mathrm{loc}}^{1}(\Omega \times D):\|\chi\|_{H^{0,1}(\Omega \times D ; M)}<\infty\right\}
$$

is a Hilbert space, it follows that $K=M \cdot H^{0,1}(\Omega \times D ; M)$, too, is a Hilbert space with inner product

$$
\left(\varphi_{1}, \varphi_{2}\right)_{K}:=\int_{\Omega \times D}\left[\frac{\varphi_{1} \varphi_{2}}{M}+M\left(\nabla_{q} \frac{\varphi_{1}}{M}\right) \cdot\left(\nabla_{q} \frac{\varphi_{2}}{M}\right)\right] \mathrm{d} q \underset{\sim}{\mathrm{d}} \underset{\sim}{x}, \quad \varphi_{1}, \varphi_{2} \in K,
$$

and induced norm $\|\varphi\|_{K}=(\varphi, \varphi)_{K}^{\frac{1}{2}}$; clearly,

$$
\|\varphi\|_{K}=\left\|\frac{\varphi}{M}\right\|_{H^{0,1}(\Omega \times D ; M)}, \quad \varphi \in K .
$$


Analogously, $K_{q}$ is a Hilbert space with norm $\|\cdot\|_{K_{q}}$ defined by

$$
\|\varphi\|_{K_{q}}^{2}=\int_{\Omega \times D}\left(1+|\underset{\sim}{q}|^{2}\right) \frac{|\varphi|^{2}}{M}+M\left|\nabla_{q}\left(\frac{\varphi}{M}\right)\right|^{2} \underset{\sim}{\mathrm{d} q} \mathrm{~d} \underset{\sim}{x}
$$

and associated inner product.

It is well-known (e.g. [9], Coro. I.2.5) that the space

$$
\underset{\sim}{W}:=\left\{\underset{\sim}{w} \in \underset{\sim}{C}(\Omega): \nabla_{x} \cdot \underset{\sim}{w}=0\right\} \quad \text { is dense in both } \underset{\sim}{V} \text { and } \underset{\sim}{H} .
$$

We require an analogous density result for the space $K$. Hypotheses $(2.18 \mathrm{a}, \mathrm{b})$ for the Maxwellian $M$ and the associated elastic potential $U$ play a crucial role in the argument.

LEMMA 3.1.

(a) Suppose that $D$ is a bounded open ball in $\mathbb{R}^{d}$ and $\kappa>0$; then, the set $M \cdot C^{\infty}(\overline{\Omega \times D})$ is dense in $K$. Here $C^{\infty}(\overline{\Omega \times D})$ denotes the set of all functions defined on $\overline{\Omega \times D}$ that are infinitely differentiable in $\Omega \times D$ and which, together with their partial derivatives of any order, can be continuously extended to $\overline{\Omega \times D}=\bar{\Omega} \times \bar{D}$.

(b) Let $D=\mathbb{R}^{d}$; then $C_{0}^{\infty}(\Omega \times D)$ is dense in $K_{q}$.

Proof. (a) For $\underset{\sim}{q} \in D$ let $d(\underset{\sim}{q}):=\operatorname{dist}(\underset{\sim}{q}, \partial D)$. According to (2.18a), there exist positive constants $c_{3}, c_{4}$, such that $c_{3} \leq M(\underset{\sim}{q}) / d^{\kappa}(\underset{\sim}{q}) \leq c_{4}$ for all $\underset{\sim}{q} \in D$. Hence the Maxwellian $M(\underset{\sim}{q})$ is a weight function of type 3 in $D$ in the sense of Triebel [22], p.247, Definition 3.2.1.3c. By [22], Theorem 3.2.2a, the weighted Sobolev space

$$
H^{1}(D ; M):=\left\{f \in L^{2}(D):\|f\|_{H^{1}(D ; M)}^{2}:=\int_{D} M\left[|f(q)|^{2}+\left|\underset{\nabla_{q}}{ } f(q)\right|^{2}\right] \underset{\sim}{\mathrm{d} q}<\infty\right\}
$$

is a Hilbert space and, by [22], Theorem 3.2.2c, $C^{\infty}(\bar{D})$ is dense in $H^{1}(D ; M)$; see also Chapter I, Section 7, in Kufner's monograph [14]. Therefore, $L^{2}\left(\Omega ; C^{\infty}(\bar{D})\right)$ is dense in $L^{2}\left(\Omega ; H^{1}(D ; M)\right)=$ $H^{0,1}(\Omega \times D ; M)$. Given $\chi \in H^{0,1}(\Omega \times D ; M)$, let $\left\{\hat{\chi}_{\varepsilon}\right\}_{\varepsilon} \subset L^{2}\left(\Omega ; C^{\infty}(\bar{D})\right)$ be a sequence which converges to $\chi$ in $H^{0,1}(\Omega \times D ; M)$. Let us extend both $\chi$ and $\widehat{\chi}_{\varepsilon}$ with respect to the variable $\underset{\sim}{x}$ by 0 outside $\Omega$ and, for $\varepsilon \in(0,1)$, define

$$
\chi_{\varepsilon}(\underset{\sim}{x} \underset{\sim}{q}):=\left(\widehat{\chi}_{\varepsilon}(\underset{\sim}{x}, \underset{\sim}{q}) \zeta_{2 \varepsilon}(\underset{\sim}{x})\right) * x j_{\varepsilon}(\underset{\sim}{x}),
$$

where $*_{x}$ denotes convolution with respect to $\underset{\sim}{x}, \zeta_{2 \varepsilon}(\underset{\sim}{x})$ is the characteristic function of the set $\Omega_{2 \varepsilon}:=\{\underset{\sim}{x} \in \Omega: \operatorname{dist}(\underset{\sim}{x}, \partial \Omega) \geq 2 \varepsilon\}$,

$$
j_{\varepsilon}(\underset{\sim}{x})=\varepsilon^{-d} j\left(\varepsilon^{-1} \underset{\sim}{x}\right),
$$

and $j$ is a nonnegative $C^{\infty}$ function with compact support, $\operatorname{supp}(j)=B(\underset{\sim}{0}, 1)$, whose integral over $\mathbb{R}^{d}$ is equal to 1 . Now, $\chi_{\varepsilon} \in C_{0}^{\infty}\left(\Omega ; C^{\infty}(\bar{D})\right)$ and the sequence $\left\{\chi_{\varepsilon}\right\}_{\varepsilon}$ converges to $\chi$ in $L^{2}\left(\Omega ; H^{1}(D ; M)\right)=H^{0,1}(\Omega \times D ; M)$ as $\varepsilon \rightarrow 0$.

For a general $\varphi \in K$, we approximate $\chi=\varphi / M \in H^{0,1}(\Omega \times D ; M)$ by a sequence $\left\{\chi_{\varepsilon}\right\}_{\varepsilon} \subset$ $C_{0}^{\infty}\left(\Omega ; C^{\infty}(\bar{D})\right) \subset C^{\infty}(\overline{\Omega \times D})$ in the $H^{0,1}(\Omega \times D ; M)$-norm. Then the sequence $\left\{M \chi_{\varepsilon}\right\}_{\varepsilon}$ converges to $M \chi=\varphi$ in the $\|\cdot\|_{K}$ norm as $\varepsilon \rightarrow 0$. 
(b) Suppose that $\varphi \in K_{q}$. On letting $\chi=\varphi / \sqrt{M}$, we deduce that

$$
\|\varphi\|_{K_{q}}^{2}=\int_{\Omega \times D}\left[\left(1+|\underset{\sim}{q}|^{2}\right)|\chi|^{2}+\left|\nabla_{q} \chi+\frac{1}{2} U^{\prime} \underset{\sim}{q} \chi\right|^{2}\right] \mathrm{d} \underset{\sim}{q} \mathrm{~d} \underset{\sim}{ } .
$$

By virtue of $(2.18 \mathrm{~b})$ in the case of $D=\mathbb{R}^{d}$, it then follows that there exist positive constants $C_{1}$ and $C_{2}$ such that

$$
C_{1}\|\varphi\|_{K_{q}}^{2} \leq \int_{\Omega \times D}\left[\left(1+|\underset{\sim}{q}|^{2}\right)|\chi|^{2}+\left|\nabla_{q} \chi\right|^{2}\right] \mathrm{d} \underset{\sim}{q} \mathrm{~d} \underset{\sim}{x} \leq C_{2}\|\varphi\|_{K_{q}}^{2} .
$$

Let us extend the functions $\varphi$ and $\chi$ with respect to the variable $\underset{\sim}{x}$ by zero outside $\Omega$, so that the extended functions, which we still denote by $\varphi$ and $\chi$, respectively, are now defined on $\mathbb{R}^{d} \times \mathbb{R}^{d}$. Let $\varepsilon \in(0,1)$ and consider the function $\varphi_{\varepsilon}=\sqrt{M} \chi_{\varepsilon}$, where

$$
\chi_{\varepsilon}(\underset{\sim}{x}, \underset{\sim}{q}):=\widehat{\chi}_{\varepsilon}(\underset{\sim}{x}, \underset{\sim}{q}) *\left(j_{\varepsilon}(\underset{\sim}{x}) j_{\varepsilon}(\underset{\sim}{q})\right) \text { and } \widehat{\chi}_{\varepsilon}(\underset{\sim}{x}, \underset{\sim}{q}):=\chi(\underset{\sim}{x}, \underset{\sim}{q}) \zeta_{2 \varepsilon}(\underset{\sim}{x}) \xi_{1 / \varepsilon}(\underset{\sim}{q}) ;
$$

here $\zeta_{2 \varepsilon}(\cdot), j(\cdot)$ are as in part (a) above, $*$ is convolution with respect to both $\underset{\sim}{x}$ and $\underset{\sim}{q}$, and

$$
\left.\xi_{1 / \varepsilon} \underset{\sim}{q}\right)=\min \left\{1,\left(1+\frac{1}{\varepsilon}-|\underset{\sim}{q}|\right)_{+}\right\}
$$

We note that the function $\xi_{1 / \varepsilon}$ has its range in the interval $[0,1]$, it is equal to 1 within the bounded open ball $B(\underset{\sim}{0}, 1 / \varepsilon) \subset \mathbb{R}^{d}$, equal to 0 in the complement of the bounded open ball $B(\underset{\sim}{0}, 1+(1 / \varepsilon)) \subset \mathbb{R}^{d}$ and $\left|\nabla_{q} \xi_{1 / \varepsilon}(\underset{\sim}{q})\right| \leq 1$ for a.e. $\underset{\sim}{q}$ in $\mathbb{R}^{d}$. The properties of the convolution imply that $\chi_{\varepsilon} \in C_{0}^{\infty}\left(\mathbb{R}^{d} \times \mathbb{R}^{d}\right)$; more precisely, $\chi_{\varepsilon} \in C_{0}^{\infty}\left(\Omega \times \mathbb{R}^{d}\right)$. Since $\sqrt{M}$ is a constant multiple of $\exp \left(-\frac{1}{2} U\right)$, and $s \in \mathbb{R} \mapsto U(s)$ is, by hypothesis, a $C^{\infty}$ function, it follows that $\sqrt{M} \in C^{\infty}\left(\mathbb{R}^{d}\right)$. Hence, $\varphi_{\varepsilon} \in C_{0}^{\infty}\left(\Omega \times \mathbb{R}^{d}\right)$.

Our aim is to show that $\lim _{\varepsilon \rightarrow 0}\left\|\varphi_{\varepsilon}-\varphi\right\|_{K_{q}}=0$. We see from (3.5) that to do so it is necessary and sufficient to prove that

$$
\lim _{\varepsilon \rightarrow 0} \int_{\mathbb{R}^{d} \times \mathbb{R}^{d}}\left[\left(1+|\underset{\sim}{q}|^{2}\right)\left|\chi_{\varepsilon}-\chi\right|^{2}+\left|\nabla_{q} \chi_{\varepsilon}-\underset{\sim}{\nabla_{q}} \chi\right|^{2}\right] \mathrm{d} \underset{\sim}{q} \mathrm{~d} \underset{\sim}{x}=0 .
$$

On recalling that $\chi_{\varepsilon}=\widehat{\chi}_{\varepsilon} *\left(j_{\varepsilon}(\underset{\sim}{x}) j_{\varepsilon}(\underset{\sim}{q})\right),(3.6)$ will in turn follow by use of a triangle inequality once we have established the following:



$$
\begin{aligned}
& \lim _{\varepsilon \rightarrow 0} \int_{\mathbb{R}^{d} \times \mathbb{R}^{d}}\left[\left(1+\underset{\sim}{|q|^{2}}\right)\left|\chi *\left(j_{\varepsilon}(\underset{\sim}{x}) \underset{\sim}{j_{\varepsilon}(q)}\right)-\chi\right|^{2}+\left|\left(\underset{\sim}{\nabla_{q}} \chi\right) *\left(j_{\varepsilon}(\underset{\sim}{x}) \underset{\sim}{j_{\varepsilon}}(\underset{\sim}{q})\right)-\underset{\sim}{\nabla_{q}} \chi\right|^{2}\right] \mathrm{d} q \underset{\sim}{\mathrm{d} x}=0 .
\end{aligned}
$$

Let us start by showing (3.7a). By Young's convolution-inequality and using that the $L^{1}$ norm of the function $\underset{\sim}{x} \underset{\sim}{q}) \mapsto j_{\varepsilon}(\underset{\sim}{x}) j_{\varepsilon}(\underset{\sim}{q})$ over $\mathbb{R}^{d} \times \mathbb{R}^{d}$ is equal to 1 , we have that

$$
\int_{\mathbb{R}^{d} \times \mathbb{R}^{d}}\left|\left(\nabla_{q} \widehat{\chi}_{\varepsilon}-\underset{\sim}{\nabla_{q}} \chi\right) *\left(j_{\varepsilon}(\underset{\sim}{x}) j_{\varepsilon}(\underset{\sim}{q})\right)\right|^{2} \mathrm{~d} \underset{\sim}{q} \mathrm{~d} \underset{\sim}{x} \leq \int_{\mathbb{R}^{d} \times \mathbb{R}^{d}}\left|\nabla_{q} \widehat{\chi}_{\varepsilon}-\underset{\sim}{\nabla_{q}} \chi\right|^{2} \mathrm{~d} \underset{\sim}{q} \mathrm{~d} \underset{\sim}{x} .
$$


Recalling the definition of $\widehat{\chi}_{\varepsilon}(\underset{\sim}{q})$, that $\left|\xi_{1 / \varepsilon}(\underset{\sim}{q})\right| \leq 1$ for all $\underset{\sim}{q}$ in $\mathbb{R}^{d}$ and that $\left|{\underset{\sim}{q}}_{q} \xi_{1 / \varepsilon}(\underset{\sim}{q})\right| \leq 1$ for a.e. $\underset{\sim}{q}$ in $\mathbb{R}^{d}$, we deduce that the right-hand side in the last inequality is bounded by

$$
2 \int_{\mathbb{R}^{d}} \int_{\mid \underset{\sim}{|q| \geq 1 / \varepsilon}}\left|\nabla_{q} \chi\right|^{2} \mathrm{~d} \underset{\sim}{q} \mathrm{~d} \underset{\sim}{x}+2 \int_{\mathbb{R}^{d}} \int_{|q| \geq 1 / \varepsilon}|\chi|^{2} \mathrm{~d} \underset{\sim}{q} \mathrm{~d} \underset{\sim}{x}+\int_{\Omega \backslash \Omega_{2 \varepsilon}} \int_{\mathbb{R}^{d}}\left|\nabla_{q} \chi\right|^{2} \mathrm{~d} \underset{\sim}{q} \mathrm{~d} \underset{\sim}{x},
$$

which converges to 0 as $\varepsilon \rightarrow 0$ by Lebesgue's dominated convergence theorem.

Analogously, by Young's inequality again, but this time applied with respect to the variable $\underset{\sim}{x}$ only, using the Cauchy-Schwarz inequality, Fubini's theorem, the change of variables $\underset{\sim}{s}=\underset{\sim}{q}-\underset{\sim}{r}$, and the inequality $1+|\underset{\sim}{s}+\underset{\sim}{r}|^{2} \leq 2\left(1+|\underset{\sim}{s}|^{2}\right)+2|\underset{\sim}{\mid r}|^{2}$, we have that

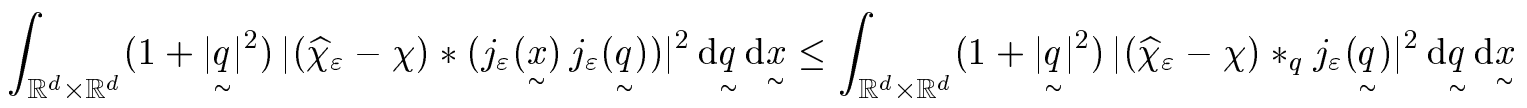

$$
\begin{aligned}
& \left.\leq \int_{\mathbb{R}^{d} \times \mathbb{R}^{d}}\left(1+|\underset{\sim}{q}|^{2}\right) \int_{|r| \leq \varepsilon} \mid \widehat{\chi}_{\varepsilon}(\underset{\sim}{x} \underset{\sim}{q}-\underset{\sim}{r})-\underset{\sim}{\left.\chi(\underset{\sim}{x}, q-\underset{\sim}{q})\right|^{2}} \underset{\sim}{j_{\varepsilon}} \underset{\sim}{r}\right) \underset{\sim}{\mathrm{d} r} \underset{\sim}{\mathrm{d} q} \mathrm{~d} \underset{\sim}{\mathrm{d} x} \\
& \left.=\int_{\mathbb{R}^{d}} \int_{|r| \leq \varepsilon} j_{\varepsilon}(\underset{\sim}{r}) \int_{\mathbb{R}^{d}}\left(1+\mid \underset{\sim}{s}+\underset{\sim}{\left.r\right|^{2}}\right) \mid \widehat{\chi}_{\varepsilon} \underset{\sim}{(x, s)}-\underset{\sim}{\chi x} \underset{\sim}{x} \underset{\sim}{s}\right)\left.\right|^{2} \mathrm{~d} \underset{\sim}{\mathrm{d}} \underset{\sim}{\mathrm{d}} \underset{\sim}{\mathrm{d} x}
\end{aligned}
$$

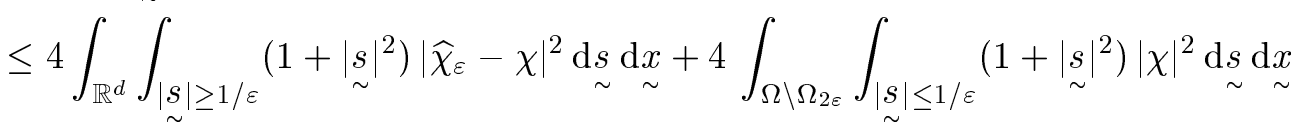

$$
\begin{aligned}
& \leq 4 \int_{\mathbb{R}^{d}} \int_{|q| \geq 1 / \varepsilon}\left(1+\underset{\sim}{|q|^{2}}\right)\left|\widehat{\chi}_{\varepsilon}-\chi\right|^{2} \underset{\sim}{\mathrm{d} q} \underset{\sim}{\mathrm{d} x}+4 \int_{\Omega \backslash \Omega_{2 \varepsilon}} \int_{\mathbb{R}^{d}}\left(1+\underset{\sim}{|q|^{2}}\right)|\chi|^{2} \mathrm{~d} \underset{\sim}{q} \mathrm{~d} x .
\end{aligned}
$$

Both integrals appearing on the right-hand side of the last inequality converge to 0 as $\varepsilon \rightarrow 0$ by Lebesgue's dominated convergence theorem. Together with (3.8), this implies (3.7a).

In order to prove $(3.7 \mathrm{~b})$, we proceed by writing

$$
\left(\nabla_{q} \chi\right) *\left(j_{\varepsilon}(\underset{\sim}{x}) j_{\varepsilon}(\underset{\sim}{q})\right)-\nabla_{q} \chi=\left(\left(\nabla_{q} \chi\right) *_{x} j_{\varepsilon}(\underset{\sim}{x})-\nabla_{q} \chi\right) *_{q} j_{\varepsilon}(\underset{\sim}{q})+\left(\left(\nabla_{q} \chi\right) *_{q} j_{\varepsilon}(\underset{\sim}{q})-\nabla_{q} \chi\right) .
$$

On applying Young's inequality with respect to $*_{q}$, we have that

$$
\begin{aligned}
& \int_{\mathbb{R}^{d} \times \mathbb{R}^{d}}\left|\left(\nabla_{q} \chi\right) *\left(j_{\varepsilon}(\underset{\sim}{x}) j_{\varepsilon}(\underset{\sim}{q})\right)-\underset{\sim}{\nabla} \chi\right|^{2} \mathrm{~d} \underset{\sim}{q} \mathrm{~d} \underset{\sim}{x} \leq 2 \int_{\mathbb{R}^{d} \times \mathbb{R}^{d}}\left|\left(\nabla_{q} \chi\right) * x j_{\varepsilon}(\underset{\sim}{x})-\nabla_{q} \chi\right|^{2} \mathrm{~d} \underset{\sim}{q} \mathrm{~d} \underset{\sim}{x} \\
& +2 \int_{\mathbb{R}^{d} \times \mathbb{R}^{d}}\left|\left(\nabla_{q} \chi\right) *_{q} j_{\varepsilon}(\underset{\sim}{q})-\nabla_{q} \chi\right|^{2} \mathrm{~d} \underset{\sim}{q} \mathrm{~d} \underset{\sim}{x} .
\end{aligned}
$$

A standard argument for Friedrichs mollifiers yields that both terms on the right-hand side of the last inequality converge to 0 as $\varepsilon \rightarrow 0$; hence,

$$
\lim _{\varepsilon \rightarrow 0} \int_{\mathbb{R}^{d} \times \mathbb{R}^{d}}\left|\left(\nabla_{q} \chi\right) *\left(j_{\varepsilon}(\underset{\sim}{x}) j_{\varepsilon}(\underset{\sim}{q})\right)-\nabla_{q} \chi\right|^{2} \mathrm{~d} \underset{\sim}{q} \mathrm{~d} \underset{\sim}{x}=0 .
$$

Finally, noting that

$$
\left|\chi *\left(j_{\varepsilon}(\underset{\sim}{x}) j_{\varepsilon}(\underset{\sim}{q})\right)-\chi\right|^{2} \leq 2\left|\left(\chi *_{q} j_{\varepsilon}(\underset{\sim}{q})-\chi\right) *_{x} j_{\varepsilon}(\underset{\sim}{x})\right|^{2}+2\left|\chi *_{x} j_{\varepsilon}(\underset{\sim}{x})-\chi\right|^{2}
$$


and applying Young's inequality with respect to $*_{x}$, we have that

$$
\begin{aligned}
& \int_{\mathbb{R}^{d} \times \mathbb{R}^{d}}\left(1+\underset{\sim}{|q|^{2}}\right)\left|\chi *\left(j_{\varepsilon}(\underset{\sim}{x}) j_{\varepsilon}(q)\right)-\chi \sim_{\sim}^{2} \mathrm{~d} \underset{\sim}{\mathrm{d}} \underset{\sim}{\mathrm{d}} \leq 2 \int_{\mathbb{R}^{d} \times \mathbb{R}^{d}}\left(1+\underset{\sim}{|q|^{2}}\right)\right| \chi *_{q} j_{\varepsilon}(\underset{\sim}{q})-\left.\chi\right|^{2} \mathrm{~d} q \underset{\sim}{\mathrm{d} x} \\
& \left.+2 \int_{\mathbb{R}^{d}} \int_{\mathbb{R}^{d}} \mid\left(1+\mid \underset{\sim}{|q|^{2}}\right)^{\frac{1}{2}} \chi *_{x} j_{\varepsilon} \underset{\sim}{x}\right)-\left.\left(1+\left.\underset{\sim}{\mid q}\right|^{2}\right)^{\frac{1}{2}} \chi\right|^{2} \underset{\sim}{\mathrm{d} x} \underset{\sim}{\mathrm{d} q} \\
& =: A_{\varepsilon}+B_{\varepsilon} \text {. }
\end{aligned}
$$

Since $\left(1+|\underset{\sim}{\mid q}|^{2}\right)^{\frac{1}{2}} \chi \in L^{2}\left(\mathbb{R}^{d} \times \mathbb{R}^{d}\right)$, a standard argument for Friedrichs mollifiers implies that

$$
\lim _{\varepsilon \rightarrow 0} B_{\varepsilon}=0 .
$$

Further, on recalling the definition of the convolution $*_{q}$, the Cauchy-Schwarz inequality, and


$2\left(\mathrm{~T}_{1, \varepsilon}+\mathrm{T}_{2, \varepsilon}\right)$, where, with $R>1$ arbitrary,

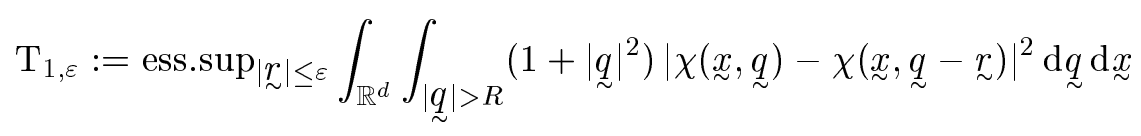

and

$$
\left.\mathrm{T}_{2, \varepsilon}:=\operatorname{ess}_{\sup } \underset{\sim}{|\underset{\sim}{\mid c}| \leq \varepsilon} \int_{\mathbb{R}^{d}} \int_{\mid \underset{\sim}{|q| \leq R}}\left(1+|\underset{\sim}{q}|^{2}\right) \mid \chi(\underset{\sim}{x} \underset{\sim}{q})-\chi \underset{\sim}{x} \underset{\sim}{q}-\underset{\sim}{r}\right)\left.\right|^{2} \mathrm{~d} \underset{\sim}{q} \mathrm{~d} \underset{\sim}{x} .
$$

Now, on using the bound $|\chi(\underset{\sim}{x}, \underset{\sim}{q})-\chi(\underset{\sim}{x}, \underset{\sim}{q} \underset{\sim}{r})| \leq 2\left(|\chi(\underset{\sim}{x}, \underset{\sim}{q})|^{2}+|\chi(\underset{\sim}{x}, \underset{\sim}{q}-\underset{\sim}{r})|^{2}\right)$, the change of variables $\underset{\sim}{s}=\underset{\sim}{q}-\underset{\sim}{r}$ and the inequality $1+\underset{\sim}{\sim} \underset{\sim}{\underline{\sim}}+\left.\underset{\sim}{ }\right|^{2} \leq 2\left(1+|\underset{\sim}{\mathcal{S}}|^{2}\right)+2|\underset{\sim}{\mid r}|^{2}$, we find that

$$
\mathrm{T}_{1, \varepsilon} \leq 10 \int_{\mathbb{R}^{d}} \int_{\mid \underset{\sim}{|q|>R-1}}\left(1+|\underset{\sim}{q}|^{2}\right)|\chi(\underset{\sim}{x}, \underset{\sim}{q})|^{2} \mathrm{~d} \underset{\sim}{q} \mathrm{~d} \underset{\sim}{x}
$$

Hence, given any $\delta>0$, there exists $R>1$, sufficiently large, such that $\mathrm{T}_{1, \varepsilon} \leq \delta / 4$. Since, for such $R>1$ fixed and $\chi \in C(\bar{\Omega} \times \overline{B(\underset{\sim}{0}, R+1)})$ we have

$$
\lim _{\varepsilon \rightarrow 0}\left\{\operatorname{ess.sup}\left|\underset{\sim}{|\mathcal{\sim}| \leq \varepsilon} \int_{\Omega} \int_{\mid \underset{\sim}{|q| \leq R}}\left(1+|\underset{\sim}{q}|^{2}\right)\right| \chi(\underset{\sim}{x} \underset{\sim}{q})-\chi\left(\underset{\sim}{x}, \underset{\sim}{q}-\left.\underset{\sim}{)}\right|^{2} \mathrm{~d} \underset{\sim}{q} \mathrm{~d} x \underset{\sim}{\sim}\right\}=0,\right.
$$

by density of $C(\bar{\Omega} \times \overline{B(0, R+1)})$ in $L^{2}(\Omega \times B(\underset{\sim}{0}, R+1))$, it then follows that the same is true for any $\chi \in L^{2}(\Omega \times B(\underset{\sim}{0}, R+1))$. Hence, there exists $\varepsilon_{0}$ such that, for all $\varepsilon \in\left(0, \varepsilon_{0}\right), \mathrm{T}_{2, \varepsilon} \leq \delta / 4$. Thus, we have shown that, for any $\delta>0$, there exists $\varepsilon_{0}>0$ such that $A_{\varepsilon} \leq 2\left(\mathrm{~T}_{1, \varepsilon}+\mathrm{T}_{2, \varepsilon}\right) \leq \delta$ for all $\varepsilon \in\left(0, \varepsilon_{0}\right)$. Hence, $\lim _{\varepsilon \rightarrow 0} A_{\varepsilon}=0$, which, together with (3.10), then implies (3.7b).

Having shown (3.7a) and (3.7b), now (3.6) follows as indicated above; hence the sequence $\left\{\varphi_{\varepsilon}\right\} \subset C_{0}^{\infty}\left(\Omega \times \mathbb{R}^{d}\right)$ converges to $\varphi \in K_{q}$ in the norm of $K_{q}$, which means that $C_{0}^{\infty}\left(\Omega \times \mathbb{R}^{d}\right)$ is dense in $K_{q}$.

Our next lemma is a trace theorem for $K$ : loosely speaking, it states that if $\varphi \in K$, then $U^{\prime}\left(\frac{1}{2}|\underset{\sim}{q}|^{2}\right) \varphi$ vanishes on $\Omega \times \partial D$ when $D$ is a bounded open ball, and decays to zero at a superalgebraic rate as $\underset{\sim}{q} \mid \rightarrow \infty$ when $D=\mathbb{R}^{d}$. 


\section{LEMMA 3.2.}

(a) Suppose that $D$ is a bounded open ball in $\mathbb{R}^{d}$ and that the elastic potential $U$ and the associated Maxwellian $M$ satisfy (2.18a) with $\kappa \geq 5$ and (2.18b). Then, for $\varphi \in K=M \cdot H^{0,1}(\Omega \times D ; M)$, the trace of $U^{\prime}\left(\frac{1}{2}|\underset{\sim}{q}|^{2}\right) \varphi$ on $\Omega \times \partial D$ is equal to 0 .

(b) Suppose that $D=\mathbb{R}^{d}$; then, for $\varphi \in K$,

$$
\lim _{R \rightarrow \infty} R^{\beta} \int_{\Omega \times \partial B(0, R)} U^{\prime}\left(\frac{1}{2}|q|^{2}\right)|\varphi| \mathrm{d} S(\underset{\sim}{q}) \mathrm{d} \underset{\sim}{x}=0 \quad \text { for all } \beta \geq 0 .
$$

Proof. (a) Let $d(q):=\operatorname{dist}(q, \partial D)$ and, for $\alpha \in \mathbb{R}$, denote by $H_{0}^{1}\left(D ; d^{\alpha}(q)\right)$ the closure of $C_{0}^{\infty}(D)$ in the $d^{\alpha}(\underset{\sim}{q})$-weighted Sobolev space $H^{1}\left(D ; d^{\alpha}(\underset{\sim}{q})\right)$; hence, $H_{0}^{1}\left(D ; d^{\alpha}(q)\right)$ is a Hilbert space with respect to the norm $\|\cdot\|_{H^{1}\left(D ; d^{\alpha}(q)\right)}$. According to a result of Besov and Kufner [2] (cf. also Triebel [22], Section 3.6.1 and Kufner [14], pp.98-99), the space $C_{0}^{\infty}(D)$ is dense in $H^{1}\left(D ; d^{\alpha}(\underset{\sim}{q})\right)$ for $\alpha \leq-1$ and $f \in H^{1}\left(D ; d^{\alpha}(\underset{\sim}{q})\right)=H_{0}^{1}\left(D ; d^{\alpha}(\underset{\sim}{q})\right)$ implies that $f=0$ on $\partial D$. Hence, to prove the Lemma, it suffices to show that if $g \in M \cdot H^{1}(D ; M)$ then $U^{\prime}\left(\frac{1}{2}|\underset{\sim}{\sim}|^{2}\right) g \in$ $H^{1}\left(D ; d^{-1}(\underset{\sim}{q})\right)$.

Let $g \in M \cdot H^{1}(D ; M)$; then,

$$
\int_{D}\left[\frac{|g|^{2}}{M}+M\left|\nabla_{q}\left(\frac{g}{M}\right)\right|^{2}\right] \mathrm{d} \underset{\sim}{q}<\infty
$$

On writing $U^{\prime} g=\left(U^{\prime} M\right) \cdot \frac{g}{M}$ and noting (2.7) and the second identity in (2.8), differentiation of $U^{\prime}\left(\frac{1}{2}|\underset{\sim}{\mid}|^{2}\right) g(\underset{\sim}{q})$ based on the product rule yields

$$
\begin{aligned}
& \left.d^{-1} \underset{\sim}{q)}\left[\left|U^{\prime} g\right|^{2}+\left.\underset{\sim}{\mid} q\left(U^{\prime} g\right)\right|^{2}\right] \leq\left(d^{-1} \underset{\sim}{q}\right)\left|U^{\prime}\right|^{2} M\right) \frac{|g|^{2}}{M} \\
& +\left(2 d^{-1} \underset{\sim}{q)} \underset{\sim}{|q|^{2}}\left|U^{\prime \prime}-\left(U^{\prime}\right)^{2}\right|^{2} M\right) \frac{|g|^{2}}{M} \\
& \left.+\left(2 d^{-1} \underset{\sim}{q}\right)\left|U^{\prime}\right|^{2} M\right)\left.M||_{\sim} \nabla_{q}\left(\frac{g}{M}\right)\right|^{2},
\end{aligned}
$$

where $U^{\prime}, U^{\prime \prime}$ and $M$ signify $U^{\prime}\left(\frac{1}{2}|\underset{\sim}{\mid}|^{2}\right), U^{\prime \prime}\left(\frac{1}{2}|\underset{\sim}{\mid}|^{2}\right)$ and $M(\underset{\sim}{q})$, respectively. Since $D$ is a bounded set, on recalling (2.18a) with $\kappa \geq 5$ and $(2.18 \mathrm{~b})$, we deduce that each of the three terms in the round brackets on the right-hand side of (3.13) is bounded on $D$; thus, by (3.12), we have that

$$
\left.\int_{D} d^{-1} \underset{\sim}{q}\right)\left[\left|U^{\prime} g\right|^{2}+\left|\underset{\sim}{\nabla}\left(U^{\prime} g\right)\right|^{2}\right] \underset{\sim}{q}<\infty .
$$

Hence, $U^{\prime}\left(\frac{1}{2}|\underset{\sim}{q}|^{2}\right) g \in H^{1}\left(D ; d^{-1}(\underset{\sim}{q})\right)$, and therefore $U^{\prime} g$ has zero trace on $\partial D$.

(b) Suppose that $D=\mathbb{R}^{d}$ and $\varphi \in K$. For $r>0$ let $B_{r}=B(\underset{\sim}{0}, r)$ be a bounded open ball of radius $r$ centred at the origin. It then follows, using the properties of $M$ and $U$ from (2.7) and $(2.18 \mathrm{a}, \mathrm{b})$ that $\varphi \in H^{0,1}\left(\Omega \times B_{r}\right):=H^{0,1}\left(\Omega \times B_{r} ; 1\right)$ and hence the trace $\left.\varphi(\underset{\sim}{x}, \cdot)\right|_{\partial B_{r}}$ exists and belongs to $L^{1}\left(\partial B_{r}\right)$ for a.e. $\underset{\sim}{x} \in \Omega$ and all $r>0$.

Let $r>0$. Any $\underset{\sim}{q} \in \partial B_{r}$ can be expressed as $\underset{\sim}{q}=r \underset{\sim}{\theta}$ where $r=|\underset{\sim}{q}|$ and $|\underset{\sim}{\theta}|=1$. Given $\beta \geq 0$, consider the function $f$ defined by

$$
f(\underset{\sim}{x}, r \underset{\sim}{\theta}):=U^{\prime}\left(\frac{1}{2} r^{2}\right) r^{d+\beta} \varphi(\underset{\sim}{x}, r \underset{\sim}{\theta}) .
$$


For every $\underset{\sim}{\theta} \in \partial B_{1}$ and a.e. $\underset{\sim}{x} \in \Omega$, held fixed, the function $r \mapsto f(\underset{\sim}{x}, r \underset{\sim}{\theta})$ belongs to $H^{1}(0, R)$ for all $R>0$; furthermore, $\left.f(\underset{\sim}{x}, \cdot)\right|_{\partial B_{r}} \in L_{1}\left(\partial B_{r}\right)$ for a.e. $\underset{\sim}{x} \in \Omega$ and all $r>0$.

Now, for a.e. $\underset{\sim}{x} \in \Omega$ and all $r \in(0, R], R>0$,

$$
\left.f \underset{\sim}{x}, R \underset{\sim}{\theta})=f \underset{\sim}{x}, r \underset{\sim}{\theta})+\int_{r}^{R} \frac{\mathrm{d}}{\mathrm{ds}} f \underset{\sim}{x}, \underset{\sim}{\theta}\right) \mathrm{d} s .
$$

Let $N(r):=\exp \left(-U\left(\frac{1}{2} r^{2}\right)\right)$; then, $N(r)=M(r \underset{\sim}{\theta})$ for all $\underset{\sim}{\theta} \in \partial B_{1}$. On re-writing

$$
f(\underset{\sim}{x}, r \underset{\sim}{\theta})=\frac{\varphi(\underset{\sim}{x}, r \underset{\sim}{\theta})}{M(r \underset{\sim}{\theta})} M(r \underset{\sim}{\theta}) U^{\prime}\left(\frac{1}{2} r^{2}\right) r^{d+\beta}=\left(\frac{\varphi(\underset{\sim}{x}, r \underset{\sim}{\theta})}{M(r \underset{\sim}{\theta})}\right) \cdot\left(N(r) U^{\prime}\left(\frac{1}{2} r^{2}\right) r^{d+\beta}\right),
$$

differentiating the product with respect to $r$ using the product rule, and defining

$$
A(r):=\sqrt{N(r)} U^{\prime}\left(\frac{1}{2} r^{2}\right) r^{\frac{1}{2}(d+1)+\beta} \quad \text { and } \quad B(r):=\frac{1}{\sqrt{N(r)}} r^{\frac{1}{2}(1-d)} \cdot \frac{\mathrm{d}}{\mathrm{d} r}\left(N(r) U^{\prime}\left(\frac{1}{2} r^{2}\right) r^{d+\beta}\right),
$$

we have from (3.14) that

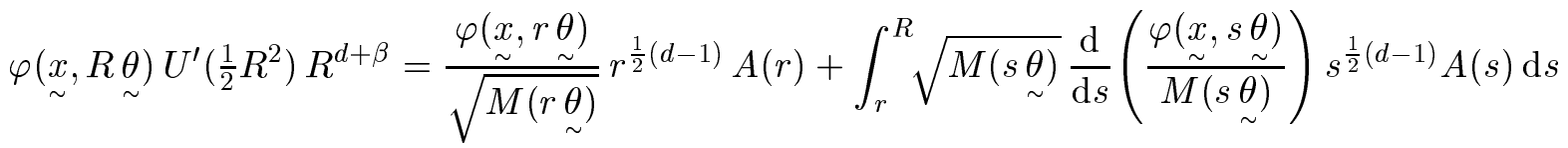

$$
\begin{aligned}
& +\int_{r}^{R} \frac{\varphi \underset{\sim}{(x, s \theta)}}{\sqrt{M(s \underset{\sim}{\sim} \boldsymbol{\sim})}} s^{\frac{1}{2}(d-1)} B(s) \mathrm{d} s
\end{aligned}
$$

Now we take the absolute value of both sides of this identity, use the triangle inequality on the right, and integrate both sides of the resulting inequality with respect to $\underset{\sim}{\theta} \in \partial B_{1}, \underset{\sim}{x} \in \Omega$ and $r \in(0, R]$, note the identity

$$
\int_{0}^{r}\left(\int_{\Omega \times \partial B_{1}} f(\underset{\sim}{x}, s \underset{\sim}{\theta}) s^{d-1} \mathrm{~d} \underset{\sim}{\theta} \mathrm{d} x\right) \mathrm{d} s=\int_{\Omega \times B_{r}} f(\underset{\sim}{x} \underset{\sim}{q}) \mathrm{d} \underset{\sim}{q} \mathrm{~d} \underset{\sim}{x}
$$

and the bound

$$
\left|\frac{\mathrm{d}}{\mathrm{d} s}\left(\frac{\varphi(\underset{\sim}{x}, s \underset{\sim}{\theta})}{M(s \underset{\sim}{\theta})}\right)\right|=\left|\left(\nabla_{q}\left(\frac{\varphi}{M}\right)(\underset{\sim}{x}, s \underset{\sim}{\theta})\right) \cdot \underset{\sim}{\theta}\right| \leq\left|\nabla_{q}\left(\frac{\varphi}{M}\right)(\underset{\sim}{x}, s \underset{\sim}{\theta})\right|
$$

and use the Cauchy-Schwarz inequality, in each of the three resulting integrals on the right, we obtain the trace inequality

$$
R^{\beta+2} U^{\prime}\left(\frac{1}{2} R^{2}\right) \int_{\Omega \times \partial B_{R}} \mid \varphi\left(\underset{\sim}{x}, \underset{\sim}{q)} \mid \mathrm{d} S \underset{\sim}{S(q)} \underset{\sim}{\mathrm{d} x} \leq C_{d}\left(C_{A}+C_{A B} R\right)\|\varphi\|_{K}\right.
$$

for all $\beta \geq 0$, where $C_{d}:=\left[\operatorname{meas}_{d}(\Omega) \cdot \operatorname{meas}_{d-1}\left(\partial B_{1}\right)\right]^{1 / 2}$, and

$$
C_{A}:=\left(\int_{0}^{R} A^{2}(r) \mathrm{d} r\right)^{\frac{1}{2}}, \quad C_{A B}:=\left(\int_{0}^{R}\left[A^{2}(r)+B^{2}(r)\right] \mathrm{d} r\right)^{\frac{1}{2}} .
$$

Finally, after dividing (3.15) by $R^{2}$, noting that $U^{\prime}\left(\frac{1}{2}|R|^{2}\right)=U^{\prime}\left(\frac{1}{2}|\underset{\sim}{q}|^{2}\right)$ for $\underset{\sim}{q} \in \partial B_{R}=\partial B(\underset{\sim}{0}, R)$, and that, under the hypotheses $(2.18 \mathrm{a}, \mathrm{b})$, we have $0<C_{A}<C_{A B}<\infty$, on passing to the limit $R \rightarrow \infty$, we obtain (3.11). 
Henceforth, we shall suppose that

$$
\kappa \geq 5 \text { when } D \text { is a bounded open ball in } \mathbb{R}^{d} .
$$

In the derivation of maximum norm bounds on $\psi$ we shall also require the following lemma.

Lemma 3.3. If $\varphi \in K_{q}$ then for any constant $L \geq 0$ it follows that

$$
\begin{aligned}
& \underset{\sim}{\nabla_{q}}\left(\frac{[\varphi-L M]_{+}}{M}\right)=\left\{\begin{array}{ll}
\nabla_{q}\left(\frac{\varphi-L M}{M}\right) \equiv \nabla_{q}\left(\frac{\varphi}{M}\right) & \text { if } \varphi>L M \\
0 & \text { if } \varphi \leq L M
\end{array},\right. \\
& \underset{\sim}{\nabla_{q}}\left(\frac{[\varphi+L M]_{-}}{M}\right)=\left\{\begin{array}{ll}
\nabla_{q}\left(\frac{\varphi+L M}{M}\right) \equiv \nabla_{q}\left(\frac{\varphi}{M}\right) & \text { if } \varphi<L M \\
0 & \text { if } \varphi \geq L M
\end{array} ;\right.
\end{aligned} ;,
$$

and hence $[\varphi-L M]_{+},[\varphi+L M]_{-} \in K_{q}$.

Proof. First, we note that as $L \geq 0$ we have that

$$
\left|[\varphi-L M]_{+}\right|,\left|[\varphi+L M]_{-}\right| \leq|\varphi| \text {. }
$$

Next, for any $\varepsilon>0$, we introduce the following regularization of $[\cdot]_{+}$:

$$
p_{+, \varepsilon}(s):=\left\{\begin{array}{ll}
\left(s^{2}+\varepsilon^{2}\right)^{\frac{1}{2}}-\varepsilon & \text { if } s \geq 0, \\
0 & \text { if } s \leq 0
\end{array} \quad \Rightarrow \quad p_{+, \varepsilon}(s) \leq[s]_{+} \quad \forall s \in \mathbb{R}\right.
$$

Then, for any fixed $\eta \in C_{0}^{\infty}(\Omega \times D)$, it follows from (3.19), (2.18a,b), the compact support of $\eta$, (2.7), $\varphi \in K$ and the Lebesgue dominated convergence theorem that

$$
\begin{aligned}
& \int_{\Omega \times D} \underset{\sim}{\nabla_{q}}\left(\frac{[\varphi-L M]_{+}}{M}\right) \cdot \underset{\sim}{\eta} \mathrm{d} q \underset{\sim}{\mathrm{d}} x=\lim _{\varepsilon \rightarrow 0} \int_{\Omega \times D} \underset{\sim}{\nabla_{q}}\left(\frac{p_{+, \varepsilon}(\varphi-L M)}{M}\right) \cdot \underset{\sim}{\eta} \underset{\sim}{\mathrm{d} q} \mathrm{~d} x \\
& =\lim _{\varepsilon \rightarrow 0} \int_{\Omega \times D}\left(p_{+, \varepsilon}^{\prime}(\varphi-L M) \frac{\underset{\nabla}{\nabla}(\varphi-L M)}{M}+p_{+, \varepsilon}(\varphi-L M) \underset{\sim}{\nabla_{q}}\left(M^{-1}\right)\right) \cdot \underset{\sim}{\eta \sim} \underset{\sim}{\mathrm{d} q} \mathrm{~d} \underset{\sim}{\sim} \\
& =\int_{\varphi>L M}\left(\frac{\underset{\sim}{\nabla_{q}(\varphi-L M)}}{M}+(\varphi-L M) \underset{\sim}{\nabla_{q}}\left(M^{-1}\right)\right) \cdot \underset{\sim}{\eta} \underset{\sim}{\mathrm{d} q} \underset{\sim}{\mathrm{d} x} \\
& =\int_{\varphi>L M} \underset{\sim}{\nabla_{q}}\left(\frac{(\varphi-L M)}{M}\right) \cdot \underset{\sim}{\eta} \underset{\sim}{\mathrm{d} q} \mathrm{~d} x \text {. }
\end{aligned}
$$

Hence we obtain the desired result (3.17a). A similar regularization of $[\cdot]_{-}$yields the desired result (3.17b). Finally (3.18), (3.17a,b) and $\varphi \in K_{q}$ imply immediately that $[\varphi-L M]_{+}$, $[\varphi+L M]_{-} \in K_{q}$.

For later purposes, we recall the following well-known Gagliardo-Nirenberg inequality. Let $r \in[2, \infty)$ if $d=2$, and $r \in[2,6]$ if $d=3$ and $\mu=d\left(\frac{1}{2}-\frac{1}{r}\right)$. Then there is a constant $C$, depending only on $\Omega, r$ and $d$, such that for all $\eta \in H_{0}^{1}(\Omega)$ the inequality

$$
\left(\int_{\Omega}|\eta|^{r} \mathrm{~d} \underset{\sim}{x}\right)^{\frac{1}{r}} \leq C\left(\int_{\Omega}|\eta|^{2} \mathrm{~d} \underset{\sim}{x}\right)^{\frac{1-\mu}{2}}\left(\int_{\Omega}|\nabla \eta|^{2} \mathrm{~d} \underset{\sim}{x}\right)^{\frac{\mu}{2}}
$$


holds.

We recall also the following compactness result, see, e.g., [21] and [19]. Let $X_{0}, X$ and $X_{1}$ be Banach spaces, $X_{i}, i=0,1$, reflexive, with a compact embedding $X_{0} \hookrightarrow X$ and a continuous embedding $X \hookrightarrow X_{1}$. Then, for $\alpha_{i}>1, i=0,1$, the embedding

$$
\left\{\eta \in L^{\alpha_{0}}\left(0, T ; X_{0}\right): \frac{\partial \eta}{\partial t} \in L^{\alpha_{1}}\left(0, T ; X_{1}\right)\right\} \hookrightarrow L^{\alpha_{0}}(0, T ; X)
$$

is compact.

\subsection{Energy identities and estimates}

The starting-point for our analysis is the following formal weak formulation of the problem: find $t \mapsto \underset{\sim}{u}(\cdot, t) \in \underset{\sim}{V}$ and $t \mapsto \psi(\cdot, t) \in K_{q}^{+}$for $t \in(0, T]$, such that

$$
\begin{aligned}
& \int_{\Omega} \frac{\partial u}{\partial t} \cdot \underset{\sim}{w} \mathrm{~d} x+\int_{\Omega}(\underset{\sim}{u} \cdot \underset{\sim}{\nabla} x) \underset{\sim}{u} \cdot \underset{\sim}{w} \mathrm{~d} x+\nu \int_{\Omega} \underset{\sim}{\nabla} x \underset{\sim}{u}: \underset{\sim}{\nabla_{x}} \underset{\sim}{w} \mathrm{~d} x \\
& =-\int_{\Omega} \underset{\approx}{\tau}(\psi): \underset{\approx}{\nabla_{x}} \underset{\sim}{w} \mathrm{~d} x \quad \forall \underset{\sim}{w} \in V, \\
& \underset{\sim}{u}(\underset{\sim}{x}, 0)=\underset{\sim}{u} \underset{\sim}{u}(x)
\end{aligned}
$$

and

$$
\begin{aligned}
& \int_{\Omega \times D} \frac{\partial \psi}{\partial t} \frac{\varphi}{M} \mathrm{~d} q \underset{\sim}{\mathrm{d} x}-\int_{\Omega \times D} \frac{\psi}{M} \underset{\sim}{u} \cdot \underset{\sim}{\nabla_{x}} \varphi \underset{\sim}{\mathrm{d} q} \underset{\sim}{\mathrm{d}} \underset{\sim}{x}+\frac{1}{2 \lambda} \int_{\Omega \times D} M \underset{\sim}{\nabla_{q}}\left(\frac{\psi}{M}\right) \cdot \underset{\sim}{\nabla_{q}}\left(\frac{\varphi}{M}\right) \underset{\sim}{\mathrm{d} q} \mathrm{~d} \underset{\sim}{\underset{\sim}{\varphi}} \\
& =\int_{\Omega \times D} \underset{\sim}{\sigma(u)} \underset{\sim}{q} \psi \cdot \underset{\sim}{\nabla_{q}}\left(\frac{\varphi}{M}\right) \underset{\sim}{\mathrm{d} q} \mathrm{~d} \underset{\sim}{\mathrm{d} x} \quad \forall \varphi \in K_{q}, \\
& \psi(\underset{\sim}{x}, \underset{\sim}{q}, 0)=\psi_{0} \underset{\sim}{(x, q)} \underset{\sim}{\sim}
\end{aligned}
$$

Here we have noted that, according to Lemma 3.2, $\varphi \in K_{q}$ implies that $\varphi$ vanishes on $\partial D$. In (3.23a), and below we use the following notation: for any $\underset{\approx}{A}, \underset{\approx}{B} \in \mathbb{R}^{d \times d}$, we define

$$
\underset{\approx}{A}: \underset{\approx}{B}:=\sum_{i=1}^{d} \sum_{j=1}^{d} \underset{\approx}{A} i j \underset{\approx}{B_{i j}} \quad \text { and } \quad|\underset{\approx}{A}|:=(\underset{\approx}{A}: \underset{\approx}{A})^{\frac{1}{2}}=\left[\operatorname{Trace}\left(\underset{\approx}{A^{\top}} \underset{\approx}{A}\right)\right]^{\frac{1}{2}} .
$$

We begin by deriving some formal energy inequalities; the purpose of these is to justify the choice of norms and spaces and indicate the kinds of bounds which will be rigorously established later on. The arguments in this section are 'formal' in the sense that some of the steps require additional smoothness of $\underset{\sim}{u}$ and $\psi$; specifically, we shall suppose throughout Section 3.2 that $\underset{\sim}{u}(\cdot, t) \in \underset{\sim}{W^{1, \infty}}(\Omega) \cap \underset{\sim}{V}$ and that $\psi(\cdot, \cdot, t) \in M \cdot H^{1}(\Omega \times D ; M) \cap K_{q}^{+}$for $t \in(0, T]$; we shall also suppose for the moment that $\underset{\sim}{u}$ and $\psi$ are sufficiently smooth in $t$. The first of these requirements will be met in Section 4 through mollification of $\underset{\sim}{u}$ as has been indicated earlier, while the requirement on $\psi$ will be relaxed to $\psi(\cdot, \cdot, t) \in K_{q}^{+}$for all $t \in(0, T]$ by considering $\frac{\partial \psi}{\partial t}+u \sim \nabla_{x} \psi$ as the space-time directional derivative (total derivative) of $\psi$ along subcharacteristic curves and rewriting this in weak form through integration by parts over $\underset{\sim}{x} \underset{\sim}{q}$ and $t$. 
First we note that

$$
\int_{\Omega}\left[(\underset{\sim}{v} \cdot \underset{\sim}{\nabla} x) \underset{\sim}{w_{1}}\right] \cdot \underset{\sim}{w_{2}} \mathrm{~d} x=-\int_{\Omega}\left[(\underset{\sim}{v} \cdot \underset{\sim}{\nabla} x) \underset{\sim}{w_{2}}\right] \cdot \underset{\sim}{w_{1}} \mathrm{~d} \underset{\sim}{x} \quad \forall \underset{\sim}{\forall v} \in V, \quad \forall w_{1}, w_{2} \in \underset{\sim}{H} H_{0}^{1}(\Omega) .
$$

Taking $\underset{\sim}{w}=\underset{\sim}{u} \in \underset{\sim}{V}$ in (3.23a) and noting (3.25) and (2.2), we obtain that

$$
\begin{aligned}
& \frac{1}{2} \frac{\mathrm{d}}{\mathrm{dt}}\left[\int_{\Omega}|u|^{2} \mathrm{~d} x\right]+\nu \int_{\Omega}\left|\underset{\approx}{\mid \nabla_{x}} \underset{\sim}{u}\right|^{2} \mathrm{~d} x=-\int_{\Omega} \underset{\sim}{\tau}(\psi): \underset{\approx}{\nabla_{x}} \underset{\sim}{u} \mathrm{~d} x=-k \mu \int_{\Omega} \underset{\sim}{C}(\psi): \underset{\approx}{\nabla_{x}} \underset{\sim}{u} \mathrm{~d} x \\
& \leq \frac{\nu}{2} \int_{\Omega}|\underset{\approx}{\nabla} \underset{\sim}{u}|^{2} \mathrm{~d} x+\frac{(k \mu)^{2}}{2 \nu} \int_{\Omega}|C(\psi)|^{2} \underset{\sim}{\mathrm{d} x}
\end{aligned}
$$

It follows from (2.3) and (2.20) that, for $\psi \in K$,

$$
\begin{aligned}
& \int_{\Omega}|C(\psi)|^{2} \underset{\sim}{\mathrm{d} x}=\int_{\Omega} \sum_{i=1}^{d} \sum_{j=1}^{d}\left(\int_{D} \psi U^{\prime} q_{i} q_{j} \underset{\sim}{\mathrm{d} q}\right)^{2} \underset{\sim}{\mathrm{d} x} \\
& \leq d\left(\left.\int_{D} M\left(U^{\prime}\right)^{2} \underset{\sim}{\mid q}\right|^{4} \underset{\sim}{\mathrm{d} q}\right)\left(\int_{\Omega \times D} \frac{|\psi|^{2}}{M} \underset{\sim}{\mathrm{d} q} \underset{\sim}{\mathrm{d} x}\right)=d \mathcal{M}\left(\int_{\Omega \times D} \frac{|\psi|^{2}}{M} \mathrm{~d} \underset{\sim}{\underset{\sim}{\mathrm{d}} \underset{\sim}{\sim})}\right) .
\end{aligned}
$$

Taking $\varphi=\psi \in M \cdot H^{1}(\Omega \times D ; M) \cap K_{q} \subset K_{q}$ in (3.24a) and noting that (2.1b,c) implies, on integration by parts, that

$$
\int_{\Omega \times D} \frac{\psi}{M} \underset{\sim}{u} \cdot \underset{\sim}{\nabla} \psi \mathrm{d} \underset{\sim}{\mathrm{d}} \mathrm{d} \underset{\sim}{x}=\frac{1}{2} \int_{\Omega \times D} \underset{\sim}{u} \cdot \underset{\sim}{\nabla} \frac{|\psi|^{2}}{M} \mathrm{~d} \underset{\sim}{q} \mathrm{~d} \underset{\sim}{x}=\frac{1}{2} \int_{\partial \Omega \times D}(\underset{\sim}{u} \cdot \underset{\sim}{n} \partial \Omega) \frac{|\psi|^{2}}{M} \mathrm{~d} \underset{\sim}{q} \mathrm{~d} \underset{\sim}{s}=0,
$$

where $n_{\partial \Omega}$ is the outward unit normal to $\partial \Omega$, we obtain that

$$
\begin{aligned}
& \frac{1}{2} \frac{\mathrm{d}}{\mathrm{dt}}\left[\int_{\Omega \times D} \frac{|\psi|^{2}}{M} \underset{\sim}{\mathrm{d} q} \underset{\sim}{\mathrm{d} x}\right]+\frac{1}{2 \lambda} \int_{\Omega \times D} M\left|\nabla_{\sim} q\left(\frac{\psi}{M}\right)\right|^{2} \underset{\sim}{\mathrm{d} q} \underset{\sim}{\mathrm{d} x} \\
& \left.=\int_{\Omega \times D} \psi \underset{\sim}{(\sigma(u)} \underset{\sim}{q}\right) \cdot \underset{\sim}{\nabla_{q}}\left(\frac{\psi}{M}\right) \mathrm{d} \underset{\sim}{\mathrm{d} x} \underset{\sim}{\sim} .
\end{aligned}
$$

Similarly to (3.27) as $\int_{D} M \mathrm{~d} \underset{\sim}{q}=1$, it follows that

$$
\int_{\Omega}|\rho(\varphi)|^{2} \mathrm{~d} \underset{\sim}{x} \leq\left(\int_{D} M \underset{\sim}{\mathrm{d} q}\right)\left(\int_{\Omega \times D} \frac{|\varphi|^{2}}{M} \underset{\sim}{\mathrm{d}} \underset{\sim}{\mathrm{d}} \underset{\sim}{x}\right)=\left(\int_{\Omega \times D} \frac{|\varphi|^{2}}{M} \mathrm{~d} \underset{\sim}{q} \underset{\sim}{\mathrm{d} x}\right) .
$$

Hence we have from (3.2a), (3.27), (3.29) and (2.2) that

$$
\varphi \in K \text { implies } \underset{\approx}{C}(\varphi), \rho(\varphi) \underset{\approx}{I} \underset{\approx}{\tau}(\varphi) \in \underset{\approx}{L^{2}}(\Omega) .
$$

The problematic term is the one appearing on the right-hand side of the energy identity (3.28). Below, we show that in the case of corotational models this term vanishes. In the noncorotational case, we introduce a different testing procedure for the Fokker-Planck equation (3.24a) so that the problematic drag term cancels with the extra-stress term in (3.26). Hence, from here on, we consider corotational and noncorotational models separately. 


\subsubsection{Corotational models}

We begin by analysing the right-hand side of (3.28) in the case when the drag term in $(2.4 \mathrm{a})$ is corotational; that is,

$$
\underset{\sim}{\sigma(u)}=-\underset{\sim}{\sigma} \underset{\sim}{\sigma}(u)]^{\top} \text { and hence } \underset{\sim}{q} \underset{\sim}{\sigma} \underset{\sim}{\sigma} \underset{\sim}{q} \underset{\sim}{q}=0 \quad \forall \underset{\sim}{q} \in \mathbb{R}^{d},
$$

corresponding to the choice $\underset{\sim}{\sigma}(\underset{\sim}{u})=\underset{\sim}{\omega}(\underset{\sim}{u})$ in $(2.6)$.

First, suppose that $D$ is a bounded open ball in $\mathbb{R}^{d}$. Given $\varphi \in K=K_{q}$, let $\left\{\varphi_{n}\right\}_{n}$ be a sequence in the space $M \cdot C^{\infty}(\overline{\Omega \times D}) \subset K=M \cdot H^{0,1}(\Omega \times D ; M)$ which converges to $\varphi$ in $K$ (cf. Lemma 3.1(a)). Then, using (3.31) with $\underset{\sim}{\sigma(u)} \underset{\sim}{\sim} \underset{\sim}{\sim}(\underset{\sim}{u}),(2.7)$ and (2.19), we deduce that

$$
\begin{aligned}
& \left.\left.\int_{\Omega \times D} \frac{\varphi_{n}}{\sqrt{M}} \underset{\sim}{(\omega)} \underset{\sim}{u} \underset{\sim}{q}\right) \cdot \sqrt{M} \underset{\sim}{\nabla_{q}}\left(\frac{\varphi_{n}}{M}\right) \underset{\sim}{\mathrm{d} q} \mathrm{~d} \underset{\sim}{\mathrm{d} x}=\frac{1}{2} \int_{\Omega \times D} M \underset{\sim}{(\omega)} \underset{\sim}{u} \underset{\sim}{q}\right) \cdot \underset{\sim}{\nabla_{q}}\left(\frac{\varphi_{n}}{M}\right)^{2} \mathrm{~d} \underset{\sim}{\mathrm{d} x} \\
& =\frac{1}{2}\left[\int_{\Omega \times \partial D}(\underset{\sim}{\omega} \underset{\sim}{u}) \underset{\sim}{q}\right) \cdot \operatorname{\sim }_{\partial D} \frac{\varphi_{n}^{2}}{M} \mathrm{~d} s \underset{\sim}{\mathrm{d}} \underset{\sim}{x}+\int_{\Omega \times D}\left(\underset{\sim}{\left(q^{\top} \underset{\sim}{\omega} \underset{\sim}{u} \underset{\sim}{q}\right)} U^{\prime} \frac{\varphi_{n}^{2}}{M} \mathrm{~d} \underset{\sim}{q} \mathrm{~d} x\right]=0 .
\end{aligned}
$$

Here in the first integral in the square bracket, we made use of the fact that $n_{\partial D}=q /\left.|q|\right|_{\partial D}$ and then applied (3.31). As $\left\{\varphi_{n} / \sqrt{M}\right\}_{n}$ and $\left\{\sqrt{M} \nabla_{q}\left(\varphi_{n} / M\right)\right\}_{n}$ converge (strongly) in $L^{2}(\Omega \times D)$ and $L_{\sim}^{2}(\Omega \times D)$ to the functions $\varphi / \sqrt{M}$ and $\sqrt{M} \nabla_{q}(\varphi / M)$, respectively, as $n \rightarrow \infty$, and $\underset{\sim}{\omega}(u) \in{\underset{\sim}{\sim}}^{\infty}(\Omega)$ by hypothesis, it follows on passing to the limit in (3.32) that

$$
\int_{\Omega \times D} \varphi(\underset{\sim}{\omega(u)} \underset{\sim}{q}) \cdot \underset{\sim}{\nabla_{q}}\left(\frac{\varphi}{M}\right) \mathrm{d} \underset{\sim}{\mathrm{d}} \underset{\sim}{x}=\int_{\Omega \times D} \frac{\varphi}{\sqrt{M}} \underset{\sim}{(\underset{\sim}{(u)} \underset{\sim}{q})} \cdot \sqrt{M} \underset{\sim}{\nabla_{q}}\left(\frac{\varphi}{M}\right) \mathrm{d} \underset{\sim}{q} \mathrm{~d} x=0 \quad \forall \varphi \in K_{q} .
$$

If, on the other hand, $D=\mathbb{R}^{d}$, then an identical argument applies by considering a sequence $\left\{\varphi_{n}\right\}$ in $C_{0}^{\infty}(\Omega \times D)$ which converges to $\varphi \in K_{q}$ (cf. Lemma 3.1(b)). In addition, as $M \in K_{q}$, the same analysis as above yields for both $D$ a bounded open ball in $\mathbb{R}^{d}$ and $D=\mathbb{R}^{d}$ that

$$
\left.\int_{\Omega \times D} M \underset{\sim}{(\omega} \underset{\sim}{u} \underset{\sim}{q}\right) \cdot \underset{\sim}{\nabla_{q}}\left(\frac{\varphi}{M}\right) \underset{\sim}{\mathrm{d} q} \underset{\sim}{\mathrm{d} x}=0 \quad \forall \varphi \in K_{q}
$$

Thus we have shown that in the corotational case, $\underset{\sim}{\sigma}(\underset{\sim}{u})=\underset{\sim}{\sim} \underset{\sim}{\sim}(u)$, identities (3.33) and (3.34) hold both when $D$ is a bounded open ball in $\mathbb{R}^{d}$ and when $D=\mathbb{R}^{d}$. This observation leads to a considerable simplification of the analysis. Indeed, upon combining (3.26), (3.27), (3.28) and (3.33) and applying a Gronwall inequality, we have that

$$
\begin{aligned}
& \sup _{t \in(0, T)}\left[\int_{\Omega \times D} \frac{|\psi|^{2}}{M} \mathrm{~d} \underset{\sim}{\mathrm{d}} \underset{\sim}{x}\right]+\frac{1}{\lambda} \int_{0}^{T}\left[\int_{\Omega \times D} M\left|\underset{\sim}{\nabla_{q}}\left(\frac{\psi}{M}\right)\right|^{2} \underset{\sim}{\mathrm{d} q} \underset{\sim}{\mathrm{d}} \underset{\sim}{x}\right] \mathrm{d} t \leq 2 \int_{\Omega \times D} \frac{\left|\psi_{0}\right|^{2}}{M} \mathrm{~d} \underset{\sim}{\mathrm{d} x} \underset{\sim}{\sim}
\end{aligned}
$$

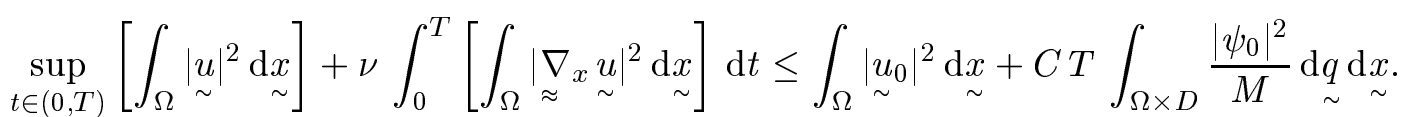

We note in passing that corotational models have a further interesting property: it follows immediately from $(2.9),(3.31)$ and (2.7) that

$$
\begin{aligned}
& \text { if } \left.\left.\psi_{0} \underset{\sim}{x} \underset{\sim}{x} q\right)=f_{0}(\underset{\sim}{x}) M(\underset{\sim}{q}) \text { then } \quad \underset{\sim}{\psi(\underset{\sim}{x}, q, t}\right)=f \underset{\sim}{(x, t)} M(\underset{\sim}{q}) \text {; } \\
& \text { where } \left.\quad \frac{\partial f}{\partial t}+\underset{\sim}{u} \cdot \underset{\sim}{\nabla} x\right) f=0 \quad \text { in } \Omega \times D \times(0, T], \quad f \underset{\sim}{f(x, 0)}=f_{0}(\underset{\sim}{x}) \quad \underset{\sim}{\forall x} \in \Omega \text {. }
\end{aligned}
$$




\subsubsection{Noncorotational models}

We now return to the physically more realistic case, $\underset{\sim}{\sigma(u)})=\gtrsim_{x} \underset{\sim}{u}$. Taking $\varphi=M \in K_{q}^{+}$in (3.24a) we obtain that

$\frac{\mathrm{d}}{\mathrm{dt}}\left[\int_{\Omega \times D} \psi \underset{\sim}{\mathrm{d} q} \underset{\sim}{\mathrm{d} x}\right]=0 \quad$ and hence $\int_{\Omega \times D} \psi \underset{\sim}{\psi(\underset{\sim}{x}, t)} \underset{\sim}{\mathrm{d} q} \underset{\sim}{\mathrm{d} x}=\int_{\Omega \times D} \psi_{0} \mathrm{~d} \underset{\sim}{\mathrm{d}} \underset{\sim}{\mathrm{d} x} \quad \forall t \in(0, T]$.

Moreover, as $\psi \in K_{q}^{+}$, it follows that

$$
\psi(\underset{\sim}{x}, \underset{\sim}{q}, t) \geq 0 \quad \text { for a.e. } \underset{\sim}{x} \underset{\sim}{q}, t) \in \Omega \times D \times(0, T] .
$$

This is, of course, a necessary condition for ensuring that $\psi$ is a probability distribution.

Taking $\varphi=M U \in K_{q}^{+}$in (3.24a), integrating by parts in the integral which is multiplied by $\frac{1}{2 \lambda}$ using (2.8), Lemma $3.2,(2.7)$ and $(2.3)$, we obtain

$$
\begin{aligned}
& \left.\frac{\mathrm{d}}{\mathrm{dt}}\left[\int_{\Omega \times D} U \psi \underset{\sim}{\mathrm{d} q} \underset{\sim}{\mathrm{d} x}\right]+\frac{1}{2 \lambda} \int_{\Omega \times D} \underset{\sim}{|q|^{2}}\left(\left(U^{\prime}\right)^{2}-U^{\prime \prime}\right)\right) \psi \underset{\sim}{\mathrm{d} q} \underset{\sim}{\mathrm{d} x} \\
& =\int_{\Omega} \underset{\approx}{\approx}(\psi): \underset{\approx}{\sigma(u)} \underset{\sim}{\mathrm{d} x}+\frac{d}{2 \lambda} \int_{\Omega \times D} U^{\prime} \psi \underset{\sim}{\mathrm{d} q} \mathrm{~d} x .
\end{aligned}
$$

On noting (2.17), $U \in C^{\infty}(D) \subset C^{2}(D),(3.38)$ and (3.37) it follows from (3.39) that

$$
\begin{aligned}
& \left.\frac{\mathrm{d}}{\mathrm{dt}}\left[\int_{\Omega \times D} U \psi \underset{\sim}{\mathrm{d} q} \mathrm{~d} \underset{\sim}{\mathrm{d} x}\right]+\frac{1}{2 \lambda} \int_{\Omega \times\left\{|q|^{2}<\frac{d}{c_{2}}\right\}} \underset{\sim}{|q|^{2}}\left(\left(U^{\prime}\right)^{2}-U^{\prime \prime}\right)\right) \psi \underset{\sim}{\mathrm{d} q} \mathrm{~d} \underset{\sim}{\mathrm{d} x}
\end{aligned}
$$

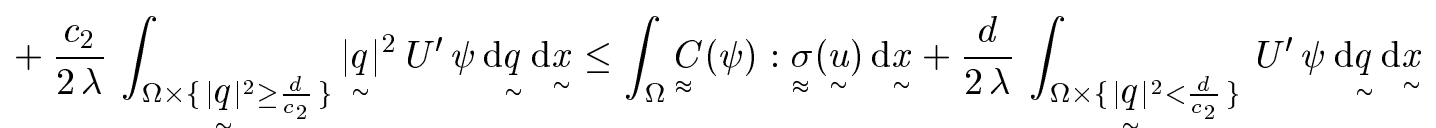

$$
\begin{aligned}
& \leq \int_{\Omega} \underset{\approx}{\approx}(\psi): \underset{\sim}{\sigma(u)} \mathrm{d} \underset{\sim}{\mathrm{d}}+C \int_{\Omega \times D} \psi_{0} \mathrm{~d} \underset{\sim}{\mathrm{d}} \underset{\sim}{\mathrm{d}} .
\end{aligned}
$$

Here, $C=\frac{d}{2 \lambda} \sup _{|\underset{\sim}{q}|^{2}<d / c_{2}} U^{\prime}\left(\frac{1}{2}|\underset{\sim}{q}|^{2}\right)<\infty$ since, by hypothesis, $B\left(\underset{\sim}{0},\left(d / c_{2}\right)^{1 / 2}\right) \subset \subset D$. In the case $\underset{\sim}{\sigma}(u)=\underset{\sim}{\nabla} \underset{\sim}{u}, \underset{\sim}{\text { on }}$ combining the first line of (3.26) and (3.40) multiplied by $k \mu$ yields that

$$
\begin{aligned}
& \frac{\mathrm{d}}{\mathrm{dt}}\left[\frac{1}{2} \int_{\Omega}|u|^{2} \mathrm{~d} \underset{\sim}{\mathrm{d} x}+k \mu \int_{\Omega \times D} U \psi \underset{\sim}{\mathrm{d} q} \underset{\sim}{\mathrm{d} x}\right]+\nu \int_{\Omega}\left|\underset{\sim}{\mid \nabla_{x}} \underset{\sim}{u}\right|^{2} \underset{\sim}{\mathrm{d} x} \\
& +\frac{k \mu c_{2}}{2 \lambda} \int_{\Omega \times\left\{|q|^{2} \geq \frac{d}{c_{2}}\right\}} \underset{\sim}{|q|^{2}} U^{\prime} \psi \underset{\sim}{\mathrm{d} q \mathrm{~d}} \underset{\sim}{x} \leq C k \mu \int_{\Omega \times D} \psi_{0} \mathrm{~d} \underset{\sim}{q \mathrm{~d} x} \sim \sim
\end{aligned}
$$

and hence that

$$
\begin{aligned}
& \frac{1}{2} \sup _{t \in(0, T)}\left[\int_{\Omega}|\underset{\sim}{\mid u}|^{2} \mathrm{~d} x\right]+k \mu \sup _{\sim \in(0, T)}\left[\int_{\Omega \times D} U \psi \underset{\sim}{\mathrm{d} q} \mathrm{~d} x\right]+\nu \int_{0}^{T}\left[\int_{\Omega}|\underset{\sim}{\mid} \underset{\sim}{x} \underset{\sim}{u}|^{2} \mathrm{~d} x\right] \mathrm{d} t \\
& +\frac{k \mu c_{2}}{2 \lambda} \int_{0}^{T}\left[\int_{\Omega \times\left\{\underset{\sim}{\left.|q|^{2} \geq \frac{d}{c_{2}}\right\}}\right.} \underset{\sim}{|q|^{2}} U^{\prime} \psi \underset{\sim}{\mathrm{d} q} \underset{\sim}{\mathrm{d} x}\right] \mathrm{d} t \leq \frac{3}{2} \int_{\Omega} \mid \underset{\sim}{\left.u_{0}\right|^{2}} \underset{\sim}{\mathrm{d} x} \\
& +3 k \mu \int_{\Omega \times D} U \psi_{0} \mathrm{~d} q \underset{\sim}{\mathrm{d} x}+3 C T k \mu \int_{\Omega \times D} \psi_{0} \mathrm{~d} \underset{\sim}{q} \mathrm{~d} x .
\end{aligned}
$$


The validity of these bounds presupposes the existence of appropriately defined weak solutions $\underset{\sim}{u}(\cdot, t) \in \underset{\sim}{V}$ and $\psi(\cdot, t) \in K_{q}^{+}, t \in(0, T]$. To make the formal bounds (3.35a,b) and (3.42) rigorous in our proof of the existence of (global-in-time) weak solutions which is based on a sequential-compactness-argument, we need to introduce some smoothing into the system; else, passage to the limit, in a sufficiently strong sense, is not warranted by the compactness argument. Therefore, ultimately, we will not prove existence of global weak solutions to the original system (P) with $\underset{\sim}{\sigma}(\underset{\sim}{)})$ given by (i) or (ii) in (2.5); but to a modified system where the velocity field $\underset{\sim}{u}$ in $(3.24 \mathrm{a})$ is appropriately mollified, and in the noncorotational case the extra-stress tensor in (3.23a). Next we describe the details of the smoothing procedure.

\subsection{Smoothing operator $\underset{\sim}{S}$}

As we have already indicated at the end of the previous section, it is necessary to introduce a 'smoothing' procedure on the velocity field $u$ in $(2.4 \mathrm{a})$ and in the noncorotational case also on the right-hand side of (2.1a). Let $\alpha>0$ be a regularization parameter. Given $\underset{\sim}{v} \in V_{\sim}^{\prime}$, the dual of $\underset{\sim}{V}$, let $\underset{\sim}{v_{\alpha}} \in \underset{\sim}{V}$ be the unique solution to the Helmholtz-Stokes problem

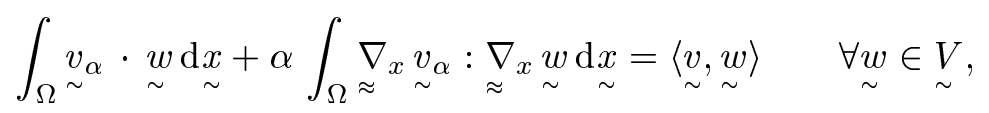

where $\langle\cdot, \cdot\rangle$ denotes the duality pairing between $\underset{\sim}{V^{\prime}}$ and $\underset{\sim}{V}$. We introduce the operator $\underset{\sim}{S}: \underset{\sim}{V^{\prime}} \rightarrow$

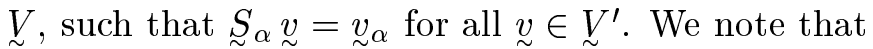

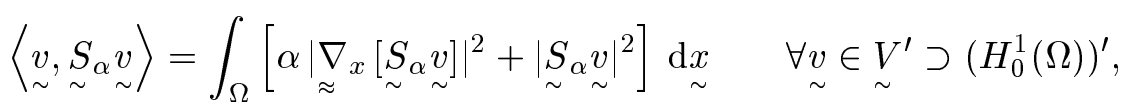

and $\left\|S_{\alpha} \cdot\right\|_{H^{1}(\Omega)}$ is a norm on $V^{\prime}$. In addition, we have from (3.43) and a Poincaré inequality that

$$
\begin{aligned}
& \left\|\underset{\sim}{S_{\alpha} v}\right\|_{L^{2}(\Omega)}^{2}+2 \alpha\left\|\underset{\sim}{\nabla_{x}} \underset{\sim}{\left[S_{\alpha} v\right]} \underset{\sim}{S_{L^{2}(\Omega)}} \leq\right\| \underset{\sim}{v} \|_{L^{2}(\Omega)}^{2} \quad \forall \underset{\sim}{v} \in \underset{\sim}{L^{2}(\Omega)} \\
& \left\|\underset{\sim}{S_{\alpha} v \|_{H^{1}(\Omega)}} \leq C\right\| \underset{\sim}{v}\left\|_{L^{2}(\Omega)}^{2} \leq C\right\| \underset{\sim}{\nabla_{x}} \underset{\sim}{v} \|_{L^{2}(\Omega)}^{2} \quad \forall \underset{\sim}{v} \in \underset{\sim}{V} .
\end{aligned}
$$

Furthermore, for $\Omega$ convex polygonal in $\mathbb{R}^{2}$ or convex polyhedral in $\mathbb{R}^{3}$ (see, respectively, [12] and [13]), or $\partial \Omega \in C^{1,1}$ in $\mathbb{R}^{d}, d=2,3$, it follows from elliptic regularity theory that

$$
\underset{\sim}{S} S_{\alpha}: \underset{\sim}{L^{2}}(\Omega) \subset \underset{\sim}{V^{\prime}} \rightarrow \underset{\sim}{V} \underset{\sim}{H_{\sim}}(\Omega) \text { is a bounded linear operator. }
$$

Moreover, for $\partial \Omega \in C^{2}$ and $r>d$ (c.f. [9, p.88]) we have that

$$
\underset{\sim}{S_{\alpha}}: \underset{\sim}{L^{r}}(\Omega) \subset \underset{\sim}{V^{\prime}} \rightarrow \underset{\sim}{V} \cap \underset{\sim}{W^{2, r}}(\Omega) \subset \underset{\sim}{V} \cap \underset{\sim}{C^{1}}(\bar{\Omega}) \quad \text { is a bounded linear operator; }
$$

and hence, we note from Sobolev embedding, elliptic regularity and a Poincaré inequality that, for $r \in(d, 6]$,

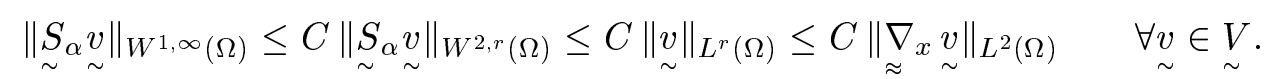

For the sake of simplicity of presentation, we shall suppose henceforth that $\partial \Omega \in C^{2}$.

Of course, other regularisation procedures could have also been used to lift the velocity field from $\underset{\sim}{V}$ to $\underset{\sim}{V} \cap \underset{\sim}{C^{1}}(\bar{\Omega})$. Our definition of $\underset{\sim}{S}$ has been motivated by the fact that, like $\underset{\sim}{u}$ itself, $S_{\alpha} u$ is defined and divergence-free on $\bar{\Omega}$, and it obeys the same boundary condition on $\tilde{\partial \Omega}$ as $u$. 


\subsection{Weak formulations}

The aim of the paper is to prove existence of a (global-in-time) solutions to each of the following weak formulations of these "smoothed" corotational and noncorotational models for any fixed regularization parameter $\alpha>0$ under the following assumptions on the data

$$
\partial \Omega \in C^{2}, \quad \sim_{0} \in \underset{\sim}{H} \quad \text { and } \quad M^{-\frac{1}{2}} \psi_{0} \in L^{2}(\Omega \times D) .
$$

Let

$$
\mathcal{K}:=\left\{\begin{array}{ll}
M \cdot C^{\infty}(\overline{\Omega \times D}) & \text { if } D \text { is a bounded open ball in } \mathbb{R}^{d} \\
C_{0}^{\infty}(\Omega \times D) & \text { if } D \equiv \mathbb{R}^{d}
\end{array} ;\right.
$$

and hence, on recalling Lemma $3.1, \mathcal{K}$ is dense in $K_{q}$. We introduce our space of test functions $\mathcal{X}$ for the $\psi$-equation as the completion of $C_{0}^{\infty}((-T, T) ; \mathcal{K})$ in the norm $\|\cdot\|_{\mathcal{X}}$ defined by

$$
\|\varphi\|_{\mathcal{X}}=\|\varphi\|_{L^{2}\left(0, T ; K_{q}\right)}+\left\|M^{-\frac{1}{2}} \frac{\partial \varphi}{\partial t}\right\|_{L^{1}\left(0 ; T ; L^{2}(\Omega \times D)\right)}+\left\|M^{-\frac{1}{2}} \nabla_{x} \varphi\right\|_{L^{1}\left(0, T ; L^{2}(\Omega \times D)\right)} .
$$

This, in particular, implies that each $\varphi \in \mathcal{X}$ satisfies $\varphi(\cdot, \cdot, T)=0$.

\section{Corotational models}

Given $T>0$, and $\Omega,{\underset{\sim}{0}}_{0}$ and $\psi_{0}$ as in $(3.48)$, find $\underset{\sim}{u} \in L^{\infty}\left(0, T ;{\underset{\sim}{L}}^{2}(\Omega)\right) \cap L^{2}(0, T ; \underset{\sim}{V}) \cap W^{1, \frac{4}{d}}\left(0, T ; V_{\sim}^{\prime}\right)$ and $\psi \in L^{2}(0, T ; K)$, with ${\underset{\sim}{\alpha}}_{\alpha}:=\underset{\sim}{S} \underset{\sim}{u} \in L^{2}\left(0, T ; \underset{\sim}{W}{ }^{1, \infty}(\Omega)\right), M^{-\frac{1}{2}} \psi \in L^{\infty}\left(0, T ; L^{2}(\Omega \times D)\right)$ and $\underset{\sim}{\tau}(\psi) \in L^{\infty}\left(0, T ; \underset{\sim}{L^{2}}(\Omega)\right)$, such that $\underset{\sim}{\sim}(\cdot, 0)={\underset{\sim}{u}}_{0}(\cdot)$, and

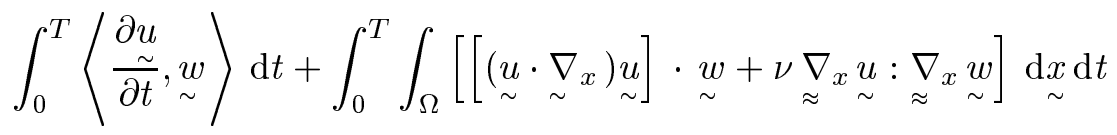

$$
\begin{aligned}
& =-k \mu \int_{0}^{T} \int_{\Omega} \underset{\approx}{\tau}(\psi): \underset{\approx}{\nabla_{x}} \underset{\sim}{w} \mathrm{~d} x \mathrm{~d} t \quad \forall \underset{\sim}{w} \in L^{\frac{4}{4-d}}(0, T ; \underset{\sim}{V}) ; \\
& \left.-\int_{0}^{T} \int_{\Omega \times D} \frac{\psi}{M}\left[\frac{\partial \varphi}{\partial t}+\underset{\sim}{\left(u_{\alpha}\right.} \cdot \underset{\sim}{\nabla} x\right) \varphi\right] \underset{\sim}{\mathrm{d} q} \underset{\sim}{\mathrm{d}} x \mathrm{~d} t-\int_{\Omega \times D} \frac{\psi_{0}(\cdot, \cdot)}{M} \varphi(\cdot, \cdot, 0) \underset{\sim}{\mathrm{d} q} \underset{\sim}{\mathrm{d} x} \\
& \left.+\int_{0}^{T} \int_{\Omega \times D}\left[\frac{M}{2 \lambda} \underset{\sim}{\nabla} q\left(\frac{\psi}{M}\right)-\underset{\sim}{[\omega} \underset{\sim}{u}\left(u_{\alpha}\right) \underset{\sim}{q}\right] \psi\right] \cdot \underset{\sim}{\nabla_{q}}\left(\frac{\varphi}{M}\right) \underset{\sim}{\mathrm{d} q} \underset{\sim}{\mathrm{d} x} \mathrm{~d} t=0 \quad \forall \varphi \in \mathcal{X} .
\end{aligned}
$$

The only difference between $(3.51 \mathrm{a}, \mathrm{b})$ and the corresponding weak formulation of the original corotational model, $(\mathrm{P})$ with $\underset{\sim}{\sigma}(\underset{\sim}{u})=\underset{\sim}{\omega}(\underset{\sim}{u})$, is that $\underset{\sim}{u}$ has been replaced by $\underset{\sim}{u}$ in $(3.51 \mathrm{~b})$.

\section{Noncorotational models}

Given $T>0$, and $\Omega,{\underset{\sim}{0}}_{0}$ and $\psi_{0}$ as in (3.48), find $\underset{\sim}{u} \in L^{\infty}\left(0, T ; L_{\sim}^{2}(\Omega)\right) \cap L^{2}(0, T ; \underset{\sim}{V}) \cap W^{1, \frac{4}{d}}\left(0, T ; V_{\sim}^{\prime}\right)$

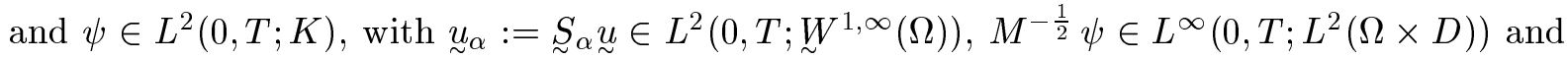


$\underset{\sim}{\tau}(\psi) \in L^{\infty}\left(0, T ; \underset{\sim}{L^{2}}(\Omega)\right)$, such that $\underset{\sim}{u}(\cdot, 0)={\underset{\sim}{u}}_{0}(\cdot)$, and

$$
\begin{aligned}
& \int_{0}^{T}\left\langle\frac{\partial u}{\partial t}, \underset{\sim}{w}\right\rangle \mathrm{d} t+\int_{0}^{T} \int_{\Omega}\left[[(\underset{\sim}{u} \underset{\sim}{\nabla} x) \underset{\sim}{u}] \cdot \underset{\sim}{w}+\nu \underset{\sim}{\nabla} \underset{\sim}{u} \underset{\sim}{u} \underset{\sim}{\nabla_{x}} \underset{\sim}{w}\right] \mathrm{d} x \mathrm{\sim} \mathrm{d} t \\
& \left.=-k \mu \int_{0}^{T} \int_{\Omega} \underset{\sim}{\tau}(\psi): \underset{\approx}{\nabla_{x}} \underset{\sim}{S_{\alpha}} \underset{\sim}{w}\right) \mathrm{d} x \mathrm{~d} t \quad \forall \underset{\sim}{w} \in L^{\frac{4}{4-d}}(0, T ; \underset{\sim}{V}) ; \\
& \left.-\int_{0}^{T} \int_{\Omega \times D} \frac{\psi}{M}\left[\frac{\partial \varphi}{\partial t}+\underset{\sim}{\left(u_{\alpha}\right.} \cdot \underset{\sim}{\nabla} x\right) \varphi\right] \underset{\sim}{\mathrm{d} q} \underset{\sim}{\mathrm{d}} \underset{\sim}{x} \mathrm{~d} t-\int_{\Omega \times D} \frac{\psi_{0}(\cdot, \cdot)}{M} \varphi(\cdot, \cdot, 0) \underset{\sim}{\mathrm{d} q} \mathrm{~d} x
\end{aligned}
$$

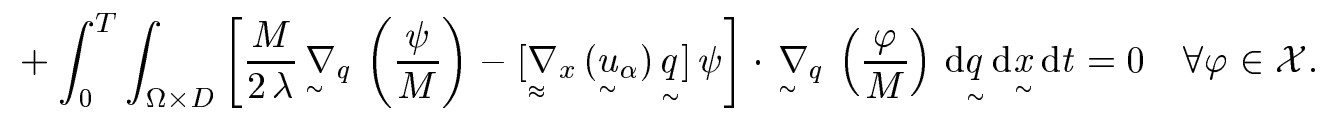

The only difference between $(3.52 \mathrm{a}, \mathrm{b})$ and $(3.51 \mathrm{a}, \mathrm{b})$ is that the corotational tensor $\underset{\sim}{\underset{\sim}{\sim}\left(u_{\alpha}\right)}$ in $(3.51 \mathrm{~b})$ is replaced by the more physical noncorotational tensor $\underset{\sim}{\nabla_{x}}\left(\underset{\sim}{u_{\alpha}}\right)$ in $(3.52 \mathrm{~b})$ and we applied smoothing on the right-hand side of $(3.52 \mathrm{a})$. Hence, $(3.52 \mathrm{a}, \mathrm{b})$ and the corresponding weak formulation of the original noncorotational model, $(\mathrm{P})$ with $\underset{\sim}{\sigma}(\underset{\sim}{u})=\underset{\sim}{\nabla} \underset{\sim}{(u)})$, differ only to the extent that $\underset{\sim}{u}$ has been replaced by $\underset{\sim}{u_{\alpha}}$ in $(3.51 \mathrm{~b})$ and we applied smoothing on the right-hand side of (3.52a).

Our energy estimate in the noncorotational case will be based on cancelling the extra-stress term on the right-hand side of (3.52a) with the drag term in (3.52b), hence mimicking the formal procedure in Section 3.2.2. To pass to the limit in (3.52b), we need to smooth $\underset{\sim}{u}$ and therefore to maintain the cancellation we need to smooth the right-hand side of (3.52a). Of course, smoothing the extra-stress tensor in (3.52a) essentially amounts to smoothing $\underset{\sim}{u}$ itself.

REMARK 3.1. Since the test functions in $\underset{\sim}{V}$ are divergence-free, the pressure has been eliminated in (3.51a) and (3.52a); it can be recovered in a very weak sense following the same procedure as for the incompressible Navier-Stokes equations discussed on p.208 in [21]; i.e., one obtains that $\int_{0}^{t} p(\cdot, s) \mathrm{d} s \in C\left([0, T] ; L^{2}(\Omega)\right)$.

REMARK 3.2. If $d=2$, then $\underset{\sim}{u} \in C([0, T] ; \underset{\sim}{H}$ ) (cf. Lemma 1.2 on p.176 of [21]), whereas if $d=3$, then $\underset{\sim}{u}$ is only weakly continuous as a mapping from $[0, T]$ into $\underset{\sim}{H}$ (similarly as in Theorem 3.1 on p.191 in [21]). It is in the latter, weaker sense that the imposition of the initial condition to the $\underset{\sim}{u}$-equation will be understood for $d=2,3$ : that is $\lim _{t \rightarrow 0}(\underset{\sim}{u}(t), \underset{\sim}{v})=(\underset{\sim}{u}, \underset{\sim}{v})$ for all $\underset{\sim}{\underset{\sim}{\sim}} \underset{\sim}{H}$.

\section{Existence}

Throughout we will assume that (2.18a,b), (2.19), (3.16) and (3.48) hold. In order to prove existence of these weak solutions to a modified version of $(\mathrm{P})$, we consider a time semidiscretization. To this end, for any $T>0$, let $N \Delta t=T$ and $t_{n}=n \Delta t, n=0 \rightarrow N$.

In order to prove existence of weak solutions under minimal smoothness requirements on the initial data, we introduce projections $\underset{\sim}{u}, \psi^{0}$ of the original initial data $\underset{\sim}{u}, \psi_{0}$, as follows:

$$
\begin{aligned}
& \stackrel{\sim}{\sim}^{0}=\underset{\sim}{S} \int_{\Delta t} u_{\sim}, \\
& \int_{\Omega \times D} M^{-1}\left(\left(1+\Delta t|q|_{\sim}^{2}\right) \psi^{0}-\psi_{0}\right) \varphi \underset{\sim}{\mathrm{d} q} \underset{\sim}{\mathrm{d} x}=0 \quad \forall \varphi \in L^{2}\left(\Omega \times D ; M^{-1}\left(1+\underset{\sim}{|q|^{2}}\right)\right) .
\end{aligned}
$$


It follows from $(4.1 \mathrm{a}, \mathrm{b})$ that ${\underset{\sim}{u}}^{0}$ converges to $\underset{\sim}{u_{0}}$ weakly in $\underset{\sim}{H}$ and $\psi^{0}$ converges to $\psi_{0}$ weakly in $L^{2}\left(\Omega \times D ; M^{-1}\right)$ as $\Delta t \rightarrow 0$.

We begin by considering the, simpler, corotational case.

\subsection{Corotational models}

We introduce a discrete-time procedure to mimic the formal energy estimate (3.26)-(3.35a,b). For $n=1 \rightarrow N$, given $\left\{u^{n-1}, \psi^{n-1}\right\} \in \underset{\sim}{V} \times K_{q}^{+}$; find $\underset{\sim}{u} \in \underset{\sim}{V}$ such that

$$
\begin{aligned}
& \int_{\Omega}\left[\frac{u^{n}-u_{\sim}^{n-1}}{\Delta t}+\left(\underset{\sim}{u^{n-1}} \cdot \underset{\sim}{\nabla} x\right){\underset{\sim}{u}}^{n}\right] \cdot \underset{\sim}{w} \mathrm{~d} x+\nu \int_{\Omega} \underset{\sim}{\nabla} \underset{\sim}{u_{\sim}^{n}}: \underset{\sim}{\nabla_{x}} \underset{\sim}{w} \mathrm{~d} x \\
& =-\int_{\Omega} \tau\left(\psi^{n-1}\right): \underset{\approx}{\nabla} \underset{\sim}{w} \underset{\sim}{\mathrm{d} x} \quad \forall \underset{\sim}{w} \in \underset{\sim}{V} .
\end{aligned}
$$

It is convenient to rewrite (4.2) as

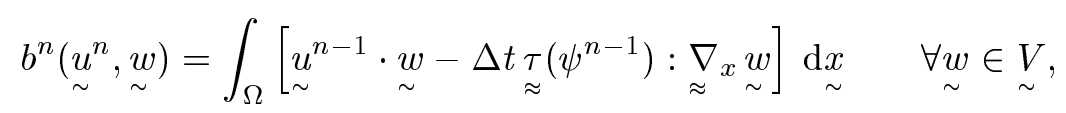

where for all $\underset{\sim}{w_{i}} \in \underset{\sim}{H}{ }_{0}^{1}(\Omega), i=1,2$,

$$
\left.b^{n}\left(\underset{\sim}{w_{1}}, \underset{\sim}{w_{2}}\right):=\int_{\Omega}\left[\underset{\sim}{w_{1}}+\Delta t \underset{\sim}{u^{n-1}} \cdot \underset{\sim}{\nabla} x\right) \underset{\sim}{w_{1}}\right] \cdot \underset{\sim}{w_{2}} \mathrm{~d} x+\Delta t \nu \int_{\Omega} \underset{\sim}{\nabla_{x}} \underset{\sim}{w_{1}}: \underset{\sim}{\nabla_{x}} \underset{\sim}{w_{2}} \mathrm{~d} x .
$$

As $u^{n-1} \in V$, it follows from $(3.25)$ that $b^{n}(\cdot, \cdot)$ is a continuous and coercive bilinear functional on $\underset{\sim}{V} \times \underset{\sim}{V}$. Since, by virtue of $(3.30), \underset{\sim}{\tau}\left(\psi^{n-1}\right) \in \underset{\sim}{L}(\Omega)$, it follows from the Lax-Milgram theorem that there exists a unique solution to (4.3).

On choosing $\underset{\sim}{w} \equiv{\underset{\sim}{u}}^{n}$ in (4.2), and noting the simple identity

$$
2\left(s_{1}-s_{2}\right) s_{1}=s_{1}^{2}+\left(s_{1}-s_{2}\right)^{2}-s_{2}^{2} \quad \forall s_{1}, s_{2} \in \mathbb{R},
$$

the identities (3.25) and (2.2), and the fact that ${\underset{\sim}{u}}^{n-1}, u_{\sim}^{n} \in \underset{\sim}{V}$ yields, similarly to (3.26), that

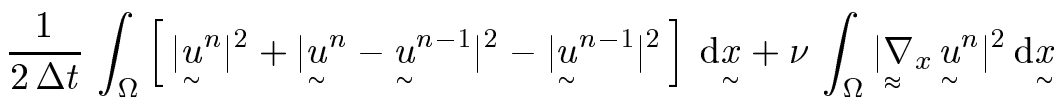

$$
\begin{aligned}
& =-k \mu \int_{\Omega} \underset{\approx}{\approx}\left(\psi^{n-1}\right): \underset{\approx}{\nabla_{x}} \underset{\sim}{u^{n}} \mathrm{~d} x \leq \frac{\sim}{2} \int_{\Omega}\left|\underset{\approx}{\nabla_{x}} \underset{\sim}{u^{n}}\right|^{2} \mathrm{~d} \underset{\sim}{x}+\frac{(k \mu)^{2}}{2 \nu} \int_{\Omega}\left|C\left(\psi^{n-1}\right)\right|^{2} \mathrm{~d} x .
\end{aligned}
$$

On choosing $\underset{\sim}{w} \equiv \underset{\sim}{S}\left(\frac{u^{n}-u^{n-1}}{\Delta t}\right) \in \underset{\sim}{V}$ in (4.2) yields, on noting (3.44), (2.2) and (3.25), that

$$
\begin{aligned}
& \int_{\Omega}\left[\alpha\left|\underset{\approx}{\nabla_{x}}\left[\underset{\sim}{S_{\alpha}}\left(\frac{\underset{\sim}{u^{n}-u^{n-1}}}{\Delta t}\right)\right]\right|^{2}+\left|\underset{\sim}{S_{\alpha}}\left(\frac{\underset{\sim}{u^{n}-u^{n-1}}}{\Delta t}\right)\right|^{2}\right] \underset{\sim}{\mathrm{d} x} \\
& =-\int_{\Omega}\left[\nu \underset{\approx}{\sim} \underset{\sim}{u_{\sim}} u_{\approx}+\left(\psi^{n-1}\right)\right]: \underset{\approx}{\nabla_{x}}\left[\underset{\sim}{S_{\alpha}}\left(\frac{u^{n}-u_{\sim}^{n-1}}{\Delta t}\right)\right] \mathrm{d} x
\end{aligned}
$$

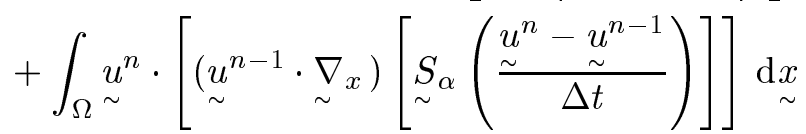

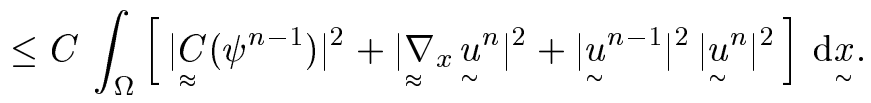


Applying the Cauchy-Schwarz inequality, the algebraic-geometric mean inequality and the Gagliardo-Nirenberg inequality (3.21) yields that

$$
\begin{aligned}
& \left.\int_{\Omega}\left|{\underset{\sim}{u}}^{n-1}\right|^{2}\left|\underset{\sim}{\left|u^{n}\right|^{2}} \underset{\sim}{\mathrm{d} x} \leq\left(\int_{\Omega} \mid \underset{\sim}{\left|u^{n-1}\right|^{4}} \underset{\sim}{\mathrm{d} x}\right)^{\frac{1}{2}}\left(\int_{\Omega} \mid \underset{\sim}{\left.u^{n}\right|^{4}} \underset{\sim}{\mathrm{d}} \underset{\sim}{x}\right)^{\frac{1}{2}} \leq \frac{1}{2} \sum_{m=n-1}^{n} \int_{\Omega}\right|{\underset{\sim}{m}}^{m}\right|^{4} \mathrm{~d} \underset{\sim}{x} \\
& \leq C \sum_{m=n-1}^{n}\left[\left(\int_{\Omega}\left|\underset{\sim}{u^{m}}\right|^{2} \mathrm{~d} x\right)^{2-\frac{d}{2}}\left(\int_{\Omega} \mid \underset{\sim}{\mid} \underset{\sim}{x} \underset{\sim}{\left.u^{m}\right|^{2} \mathrm{~d} x}\right)^{\frac{d}{2}}\right] \text {. }
\end{aligned}
$$

Given $\underset{\sim}{v} \in \underset{\sim}{V} \cap{\underset{\sim}{0,1}}^{0,1}(\bar{\Omega})$, let ${\underset{\sim}{n}}^{n}(\underset{\sim}{v}, t ; \cdot, \cdot) \in C^{1}\left(\left[t_{n-1}, t_{n}\right] ;{\underset{\sim}{C}}^{0,1}(\bar{\Omega})\right)$ be the unique solution, for all $x \in \bar{\Omega}$ and with either $t=t_{n-1}$ or $t_{n}$, of

$$
\left.\left.\left.\frac{\mathrm{d}}{\mathrm{ds}} y_{\sim}^{n}(\underset{\sim}{v}, t ; \underset{\sim}{x}, s)=\underset{\sim}{v} \underset{\sim}{y^{n}} \underset{\sim}{v}, t ; \underset{\sim}{x}, s\right)\right) \quad \forall s \in\left[t_{n-1}, t_{n}\right] \backslash t, \quad \underset{\sim}{y^{n}} \underset{\sim}{v}, t ; \underset{\sim}{x}, t\right)=\underset{\sim}{x} .
$$

As $\underset{\sim}{v}=\underset{\sim}{0}$ on $\partial \Omega$, the map $\underset{\sim}{x} \rightarrow{\underset{\sim}{n}}^{n}(\underset{\sim}{v}, t ; \underset{\sim}{x}, s)$ is, for each $s \in\left[t_{n-1}, t_{n}\right]$ and $t=t_{n-1}$ or $t_{n}$, a Lipschitz-continuous homeomorphism from $\bar{\Omega}$ into itself, independent of the choice of $\Delta t$. By virtue of the Rademacher-Stepanov theorem it is differentiable almost everywhere in $\Omega$. Moreover, since $\underset{\sim}{v}$ is divergence-free, the map has the volume-preserving property, i.e. its Jacobian, $\operatorname{det} \underset{\approx}{\nabla_{x}}{\underset{\sim}{n}}^{n}$, satisfies

$$
\left.\operatorname{det} \underset{\approx}{\nabla} \underset{\sim}{y^{n}} \underset{\sim}{v}, t ; \cdot, \cdot\right)=1 \quad \text { a.e. in } \Omega \times\left[t_{n-1}, t_{n}\right]
$$

We note also from $(4.9)$ that, for all $\underset{\sim}{x} \in \bar{\Omega}$,

$$
\begin{aligned}
& \left.\mid \underset{\sim}{\mid y^{n}} \underset{\sim}{v}, t_{n-1} ; \underset{\sim}{x}, t\right)-\underset{\sim}{x}\left|\leq\left(t-t_{n-1}\right) \sup _{y \in \Omega} \underset{\sim}{v} \underset{\sim}{v(y)}\right| \quad \forall t \in\left[t_{n-1}, t_{n}\right] \\
& \left.\left.\mid \underset{\sim}{y^{n}}\left(\underset{\sim}{v}, t_{n-1} ; \underset{\sim}{x}, t_{n}\right)-[\underset{\sim}{x}+\Delta t \underset{\sim}{v} \underset{\sim}{x})\right]|=| \int_{t_{n-1}}^{t_{n}}\left[\underset{\sim}{v}(\underset{\sim}{y^{n}} \underset{\sim}{v}, \underbrace{}_{n-1} ; \underset{\sim}{x}, t)\right)-\underset{\sim}{v} \underset{\sim}{v}(x)\right] \mathrm{d} t \mid \leq C\left(\|\underset{\sim}{v}\|_{C^{0, \frac{1}{2}}(\bar{\Omega})}\right)(\Delta t)^{\frac{3}{2}} \\
& \leq C\left(\|\|_{\sim}^{v} \|_{H^{2}(\Omega)}\right)(\Delta t)^{\frac{3}{2}} \quad \text { if } \underset{\sim}{v} \in H^{2}(\Omega) .
\end{aligned}
$$

In addition, it is easily established that, for all $t \in\left[t_{n-1}, t_{n}\right]$, all $\underset{\sim}{x} \in \bar{\Omega}$ and all ${\underset{\sim}{v}}_{a},{\underset{\sim}{b}}_{b}$ in $C^{0,1}(\bar{\Omega})$,

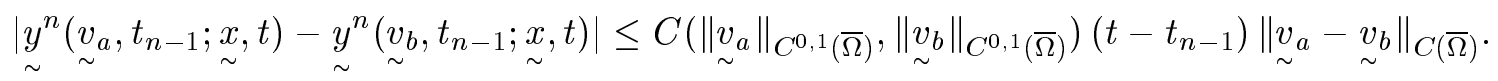

On observing that, by (3.47a), ${\underset{\sim}{\alpha}}_{\alpha}^{n}:=\underset{\sim}{S} u_{\sim}^{n} \in C^{1}(\bar{\Omega})$, we let $\psi^{n} \in K_{q}$ be such that

$$
a_{c}^{n}\left(\psi^{n}, \varphi\right)=\ell_{c}^{n}(\varphi) \quad \forall \varphi \in K_{q}
$$

where, for all $\varphi_{1}, \varphi_{2}, \varphi \in K_{q}$,

$$
\begin{aligned}
& a_{c}^{n}\left(\varphi_{1}, \varphi_{2}\right):=\int_{\Omega \times D}\left(W_{c} \varphi_{1} \varphi_{2}+\Delta t\left[\frac{M}{2 \lambda} \underset{\sim}{\nabla_{q}}\left(\frac{\varphi_{1}}{M}\right)-\underset{\sim}{\left[\omega\left(u_{\alpha}^{n}\right) \underset{\sim}{q}\right]} \varphi_{1}\right] \cdot \underset{\sim}{\nabla_{q}}\left(\frac{\varphi_{2}}{M}\right)\right) \mathrm{d} q \underset{\sim}{\mathrm{d} x}, \\
& \left.\left.\ell_{c}^{n}(\varphi):=\int_{\Omega \times D} W_{c} \psi^{n-1} \underset{\sim}{\left(y^{n}\right.} \underset{\sim}{u_{\alpha}^{n}}, t_{n} ; \underset{\sim}{x}, t_{n-1}\right), \underset{\sim}{q}\right) \varphi \underset{\sim}{\operatorname{d}} \underset{\sim}{\mathrm{d} x}, \\
& W_{c}:=\frac{1+\Delta t \underset{\sim}{|q|^{2}}}{M} \text {. }
\end{aligned}
$$




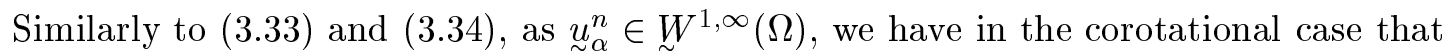

$$
\int_{\Omega \times D} \varphi\left(\underset{\approx}{\omega}\left(u_{\sim}^{n}\right) \underset{\sim}{q}\right) \cdot \underset{\sim}{\nabla} q\left(\frac{\varphi}{M}\right) \underset{\sim}{\mathrm{d} q} \underset{\sim}{\mathrm{d} x}=0, \quad \int_{\Omega \times D} M \underset{\sim}{\left(\underset{\sim}{\omega}\left(u_{\alpha}^{n}\right) \underset{\sim}{q}\right) \cdot \underset{\sim}{\nabla} q}\left(\frac{\varphi}{M}\right) \underset{\sim}{\mathrm{d} q} \underset{\sim}{\mathrm{d} x}=0 \quad \forall \varphi \in K_{q} .
$$

Hence $a_{c}^{n}(\cdot, \cdot)$ is a non-symmetric, continuous and coercive bilinear functional on $K_{q} \times K_{q}$ and, on noting (4.10), $\ell_{c}^{n}(\cdot)$ is a continuous linear functional on $K_{q}$. Therefore, the Lax-Milgram theorem yields the existence of a unique solution to (4.13). As $\psi^{n} \in K_{q}$ implies that $\left[\psi^{n}\right]_{-} \in K_{q}$, recall Lemma 3.3 , and as $\psi^{n-1} \in K_{q}^{+}$, it follows from $(3.17 \mathrm{~b}),(4.14 \mathrm{a}, \mathrm{b})$ and (4.13) that

$$
a_{c}^{n}\left(\left[\psi^{n}\right]_{-},\left[\psi^{n}\right]_{-}\right)=a_{c}^{n}\left(\psi^{n},\left[\psi^{n}\right]_{-}\right)=\ell_{c}^{n}\left(\left[\psi^{n}\right]_{-}\right) \leq 0 .
$$

Therefore the coercivity of $a_{c}^{n}(\cdot, \cdot)$ over $K_{q} \times K_{q}$ yields that $\left[\psi^{n}\right]_{-}=0$; that is, $\psi^{n} \in K_{q}^{+}$, $n=0 \rightarrow N$.

Choosing $\varphi=\psi^{n}$ in (4.13), noting (4.5), (4.15) and (4.10) yield that

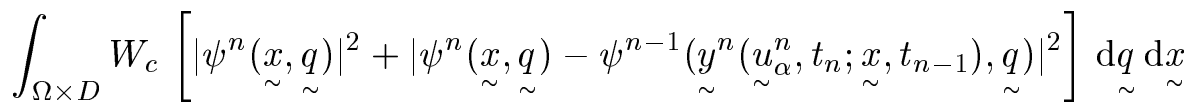

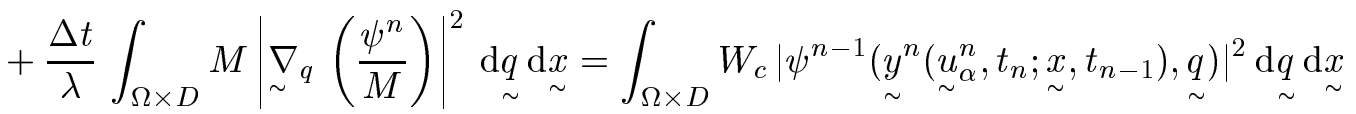

$$
\begin{aligned}
& =\int_{\Omega \times D} W_{c}\left|\psi^{n-1} \underset{\sim}{x, q} \underset{\sim}{q}\right|^{2} \underset{\sim}{\mathrm{d} q} \mathrm{~d} x .
\end{aligned}
$$

Summing (4.17) and (4.6) multiplied by $2 \Delta t$ from $n=1 \rightarrow m$, with $1 \leq m \leq N$, and noting (3.27) yields the analogues of the formal energy bounds $(3.35 \mathrm{a}, \mathrm{b})$ :

$$
\begin{aligned}
& \max _{n=1 \rightarrow N}\left[\int_{\Omega \times D} W_{c}\left|\psi^{n}\right|^{2} \underset{\sim}{\mathrm{d} q} \mathrm{~d} \underset{\sim}{x}\right]+\frac{1}{\lambda} \sum_{n=1}^{N} \Delta t \int_{\Omega \times D} M\left|\underset{\sim}{\nabla_{q}}\left(\frac{\psi^{n}}{M}\right)\right|^{2} \underset{\sim}{\mathrm{d} q} \underset{\sim}{\mathrm{d} x} \\
& \left.\left.+\sum_{n=1}^{N} \int_{\Omega \times D} W_{c} \mid \psi^{n} \underset{\sim}{\underset{\sim}{x} \underset{\sim}{q})}-\psi^{n-1} \underset{\sim}{\left(y^{n}\right.} \underset{\sim}{\left(u_{\alpha}^{n}\right.}, t_{n} ; \underset{\sim}{x}, t_{n-1}\right) \underset{\sim}{q}\right)\left.\right|^{2} \mathrm{~d} \underset{\sim}{q} \mathrm{~d} x \\
& +\max _{n=0 \rightarrow N}\left[\int_{\Omega}\left|C\left(\psi^{n}\right)\right|^{2} \mathrm{~d} x\right] \leq C \int_{\Omega \times D} W_{c}\left|\psi^{0}\right|^{2} \underset{\sim}{\mathrm{d} q} \mathrm{~d} \underset{\sim}{\mathrm{d} x} ;
\end{aligned}
$$

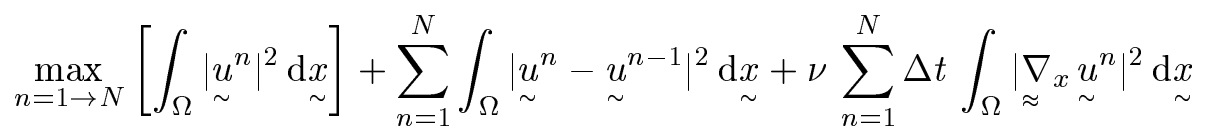

$$
\begin{aligned}
& \leq \int_{\Omega}\left|\underset{\sim}{u^{0}}\right|^{2} \mathrm{~d} \underset{\sim}{x}+C T \int_{\Omega \times D} W_{c}\left|\psi^{0}\right|^{2} \underset{\sim}{\mathrm{d} q} \mathrm{~d} x .
\end{aligned}
$$

In addition, taking the $\frac{2}{d}$ power of both sides of (4.7), summing from $n=1 \rightarrow N$ and noting 
(4.8) and $(4.18 \mathrm{a}, \mathrm{b})$ yields that

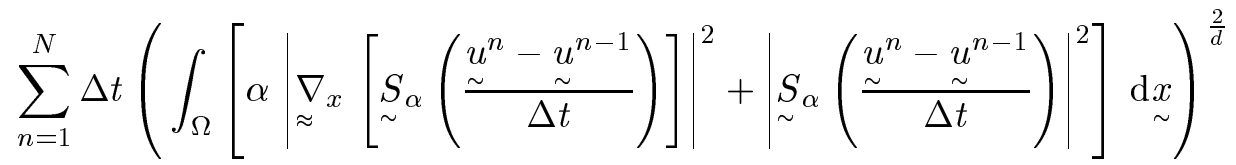

$$
\begin{aligned}
& \leq C\left[\sum_{n=1}^{N} \Delta t\left(\int_{\Omega}\left|C \underset{\approx}{\mid}\left(\psi^{n-1}\right)\right|^{2} \mathrm{~d} \underset{\sim}{x}\right)^{\frac{2}{d}}\right]+C(T)\left[\sum_{n=1}^{N} \Delta t \int_{\Omega}\left|\underset{\approx}{\underset{\sim}{u_{\sim}}} \underset{\sim}{n}\right|^{2} \underset{\sim}{\mathrm{d} x}\right]^{\frac{2}{d}} \\
& +C(T)\left[\max _{n=0 \rightarrow N}\left(\int_{\Omega}\left|\sim_{\sim}^{n}\right|^{2} \mathrm{~d} x\right)^{\frac{4}{d}-1}\right]\left[\sum_{n=0}^{N} \Delta t \int_{\Omega}\left|\underset{\sim}{\mid} \underset{\sim}{u_{\sim}^{n}}\right|^{2} \mathrm{~d} x\right] \leq C(T) ;
\end{aligned}
$$

provided that there exists a positive constant $C$, independent of $\Delta t$, such that the projected initial data satisfies

$$
\int_{\Omega}\left[\left|u_{\sim}^{0}\right|^{2}+\Delta t \underset{\sim}{\nabla_{x}} \underset{\sim}{\left.\left.u^{0}\right|^{2}\right]} \underset{\sim}{\mathrm{d} x}+\int_{\Omega \times D}\left(1+\Delta t \underset{\sim}{|q|^{2}}\right) \frac{\left|\psi^{0}\right|^{2}}{M} \mathrm{~d} \underset{\sim}{q} \underset{\sim}{\mathrm{d} x} \leq C .\right.
$$

This is guaranteed by (3.45a), (3.48) and (4.1a,b). Furthermore, it follows from (4.18b), (3.46) and (4.20) that

$$
\max _{n=0 \rightarrow N}\left\|u_{\sim}^{n}\right\|_{H^{2}(\Omega)}^{2} \leq C\left[\int_{\Omega}\left|{\underset{\sim}{0}}^{0}\right|^{2} \mathrm{~d} x+T \int_{\Omega \times D} W_{c}\left|\psi^{0}\right|^{2} \underset{\sim}{\mathrm{d} q} \mathrm{~d} \underset{\sim}{x}\right] \leq C(T) .
$$

Let

$$
\stackrel{\sim}{\sim}^{\Delta t}(\cdot, t):=\frac{t-t_{n-1}}{\Delta t}{\underset{\sim}{u}}^{n}(\cdot)+\frac{t_{n}-t}{\Delta t}{\underset{\sim}{u}}^{n-1}(\cdot), \quad t \in\left[t_{n-1}, t_{n}\right], \quad n \geq 1,
$$

and

$$
\stackrel{\sim}{u}^{\Delta t,+}(\cdot, t):={\underset{\sim}{u}}^{n}(\cdot), \quad \sim_{\sim}^{\Delta t,-}(\cdot, t):=\sim^{n-1}(\cdot), \quad t \in\left(t_{n-1}, t_{n}\right], \quad n \geq 1 .
$$

We note for future reference that

$$
\stackrel{\sim}{u}^{\Delta t}-{\underset{\sim}{u}}^{\Delta t, \pm}=\left(t-t_{n}^{ \pm}\right) \frac{\partial \tilde{u}^{\Delta t}}{\partial t}, \quad t \in\left(t_{n-1}, t_{n}\right), \quad n \geq 1,
$$

where $t_{n}^{+}:=t_{n}$ and $t_{n}^{-}:=t_{n-1}$. Using the above notation, and introducing analogous notation for $\left\{u_{\sim}^{n}\right\}_{n=0}^{N}$ and $\left\{\psi^{n}\right\}_{n=0}^{N}$, (4.2) summed for $n=1 \rightarrow N$ can be restated as:

$$
\begin{aligned}
& \int_{0}^{T}\left\langle\frac{\partial u_{\sim}^{\Delta t}}{\partial t}, \underset{\sim}{w}\right\rangle \mathrm{d} t+\int_{0}^{T} \int_{\Omega}\left[\left[\left(u_{\sim}^{\Delta t,-} \cdot \underset{\sim}{\nabla} x\right) \underset{\sim}{u^{\Delta t,+}}\right] \cdot \underset{\sim}{w}+\underset{\sim}{\nu} \underset{\sim}{u_{\sim}} u^{\Delta t,+}: \underset{\sim}{\nabla_{x}} \underset{\sim}{w}\right] \underset{\sim}{\mathrm{d}} x \mathrm{~d} t \\
& =-k \mu \int_{0}^{T} \int_{\Omega} \underset{\sim}{\tau}\left(\psi^{\Delta t,-}\right): \underset{\approx}{\nabla_{x}} \underset{\sim}{w} \mathrm{~d} x \mathrm{\sim} \mathrm{d} t \quad \forall \underset{\sim}{w} \in L^{\frac{4}{4-d}}(0, T ; \underset{\sim}{V}) .
\end{aligned}
$$

Similarly, (4.13) summed for $n=1 \rightarrow N$ can be restated as:

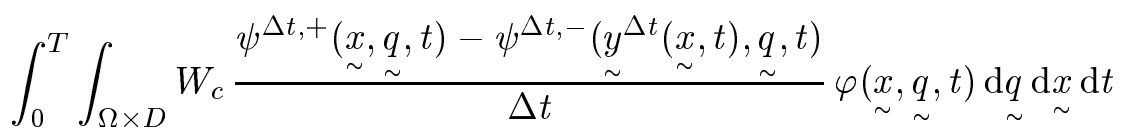

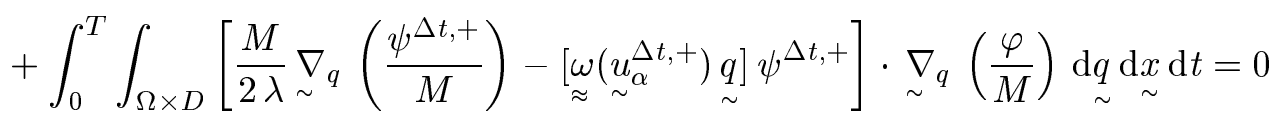

$$
\begin{aligned}
& \forall \varphi \in L^{2}\left(0, T ; K_{q}\right) \text {; }
\end{aligned}
$$


where

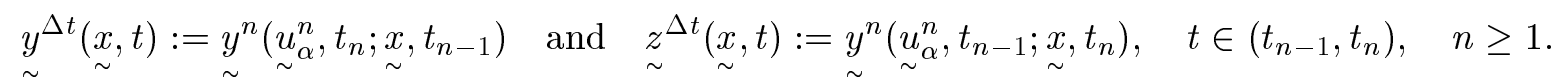

Noting (4.20), (2.2) and (3.29), we have from (4.18a) that

$$
\begin{aligned}
& \sup _{t \in(0, T)}\left[\int_{\Omega \times D} \frac{\mid \psi^{\Delta t(, \pm)} \underset{\sim}{x, \underset{\sim}{q}, t)\left.\right|^{2}}}{M} \mathrm{~d} \underset{\sim}{\underset{\sim}{\mathrm{d}} \underset{\sim}{\sim}]}\right]+\frac{1}{\lambda} \int_{0}^{T} \int_{\Omega \times D} M\left|\underset{\sim}{\nabla_{q}}\left(\frac{\psi^{\Delta t(, \pm)}}{M}\right)\right|^{2} \underset{\sim}{\mathrm{d}} \underset{\sim}{\mathrm{d} x} \mathrm{~d} t
\end{aligned}
$$

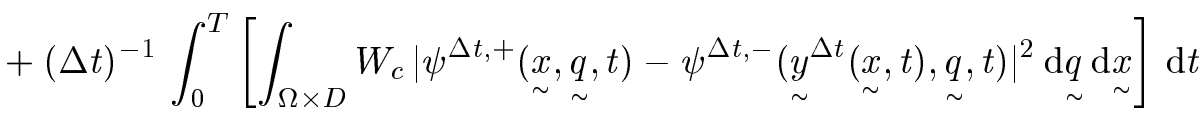

$$
\begin{aligned}
& +\sup _{t \in(0, T)}\left[\int_{\Omega}\left|\left[\underset{\approx}{\tau}\left(\psi^{\Delta t(, \pm)}\right)\right] \underset{\sim}{x, t)}\right|^{2} \mathrm{~d} x\right] \leq C .
\end{aligned}
$$

In the above, the notation $\psi^{\Delta t(, \pm)}$ means $\psi^{\Delta t}$ with or without the superscripts \pm . Similarly, on noting (4.20), we have from (4.18b), (4.19) and (4.21) that

$$
\begin{aligned}
& \sup _{t \in(0, T)}\left[\int_{\Omega}\left|u_{\sim}^{\Delta t(, \pm)}\right|^{2} \mathrm{~d} x\right]+(\Delta t)^{-1} \int_{0}^{T} \int_{\Omega}\left|{\underset{\sim}{u}}^{\Delta t,+}-{\underset{\sim}{u}}^{\Delta t,-}\right|^{2} \underset{\sim}{\mathrm{d} x} \mathrm{~d} t+\nu \int_{0}^{T} \int_{\Omega}\left|\underset{\approx}{\mid} \underset{\sim}{u_{\sim}} u^{\Delta t,( \pm)}\right|^{2} \mathrm{~d} \underset{\sim}{x} \\
& +\int_{0}^{T}\left\|\underset{\sim}{S} \frac{\partial u^{\Delta t}}{\partial t}\right\|_{H^{1}(\Omega)}^{\frac{4}{d}} \mathrm{~d} t+\sup _{t \in(0, T)}\left\|\underset{\sim}{u_{\alpha}^{\Delta t(, \pm)}}\right\|_{H^{2}(\Omega)}^{2} \leq C .
\end{aligned}
$$

We are now in a position to prove the following convergence result.

LEMMA 4.1. There exists a subsequence of $\left\{{\underset{\sim}{u}}^{\Delta t}, \psi^{\Delta t}\right\}_{\Delta t}$, and functions $\underset{\sim}{u} \in L^{\infty}\left(0, T ; L_{\sim}^{2}(\Omega)\right) \cap$ $L^{2}(0, T ; V) \cap W^{1, \frac{4}{d}}\left(0, T ; V_{\sim}^{\prime}\right)$ and $\psi \in L^{2}(0, T ; K)$ with $M^{-\frac{1}{2}} \psi \in L^{\infty}\left(0, T ; L^{2}(\Omega \times D)\right)$ such that as $\Delta t \rightarrow 0$,

$$
\begin{aligned}
& \frac{\psi^{\Delta t(, \pm)}}{M^{\frac{1}{2}}} \rightarrow \frac{\psi}{M^{\frac{1}{2}}} \quad \text { weak * in } L^{\infty}\left(0, T ; L^{2}(\Omega \times D)\right), \\
& M^{\frac{1}{2}} \underset{\sim}{\nabla_{q}}\left(\frac{\psi^{\Delta t,+}}{M}\right) \rightarrow M_{\sim}^{\frac{1}{2}} \underset{\sim}{\nabla_{q}}\left(\frac{\psi}{M}\right) \quad \text { weakly in } L^{2}\left(0, T ; \underset{\sim}{L^{2}}(\Omega \times D)\right) \text {, } \\
& \underset{\approx}{\tau\left(\psi^{\Delta t(, \pm)}\right) \rightarrow \underset{\approx}{\tau}(\psi) \quad \text { weak }}{ }^{*} \text { in } L^{\infty}\left(0, T ; \underset{\approx}{L^{2}}(\Omega)\right) ;
\end{aligned}
$$

and

$$
\begin{aligned}
& u^{\Delta t(, \pm)} \rightarrow \underset{\sim}{u} \quad \text { weak * in } L^{\infty}\left(0, T ; L_{\sim}^{2}(\Omega)\right), \\
& \stackrel{\sim}{\sim}^{\Delta t(, \pm)} \rightarrow \underset{\sim}{u} \quad \text { weakly in } L^{2}(0, T ; \underset{\sim}{V}), \\
& \underset{\sim}{S_{\alpha}} \frac{\partial u^{\Delta t}}{\partial t} \rightarrow \underset{\sim}{S_{\alpha}} \frac{\partial u}{\partial t} \quad \text { weakly in } L^{\frac{4}{d}}(0, T ; \underset{\sim}{V}) \text {, } \\
& u_{\sim}^{u^{\Delta t(, \pm)}} \rightarrow \underset{\sim}{u} \quad \text { strongly in } L^{2}\left(0, T ; \underset{\sim}{L^{r}}(\Omega)\right) \text {, } \\
& \underset{\sim}{u_{\alpha}^{\Delta t(, \pm)}} \rightarrow \underset{\sim}{u_{\alpha}}:=\underset{\sim}{S} S_{\alpha} u \quad \text { strongly in } L^{2}\left(0, T ; \underset{\sim}{W^{2, r}}(\Omega)\right) \text {, }
\end{aligned}
$$

where $r \in[1, \infty)$ if $d=2$ and $r \in[1,6)$ if $d=3$. 
PROOF. The result $(4.29 \mathrm{a})$ for $\psi^{\Delta t,+}(\underset{\sim}{x}, \underset{\sim}{q}, t)$ and $\psi^{\Delta t,-}\left(\underset{\sim}{y^{\Delta t}}(\underset{\sim}{x}, \underset{\sim}{q}), \underset{\sim}{q}, t\right)$ follow immediately from the bounds on the first and the third term on the left-hand side of (4.27) on noting (4.14c). Next we note from (4.26), (4.10), the bound on the first term on the left-hand side of (4.27) and $(4.11 \mathrm{a}, \mathrm{b})$ that, for a.a. $t \in(0, T)$,

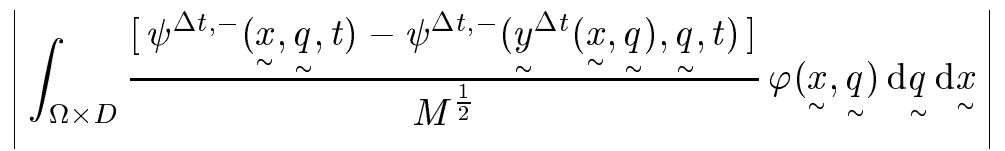

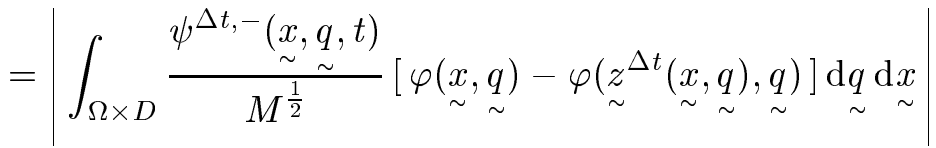

$$
\begin{aligned}
& \leq C \Delta t\left\|u_{\alpha}^{\Delta t,+}(\cdot, t)\right\|_{H^{2}(\Omega)}\|\varphi\|_{C^{0,1}(\overline{\Omega \times D})} \quad \forall \varphi \in C_{0}^{\infty}(\Omega \times D) .
\end{aligned}
$$

Hence, the desired result in (4.29a) for $\psi^{\Delta t,-}$ follows from (4.31), the bound on the fifth term on the left-hand side of (4.28) and the denseness of $C_{0}^{\infty}(\Omega \times D)$ in $L^{2}(\Omega \times D)$. The desired result in (4.29a) for $\psi^{\Delta t}$ then follows from that for $\psi^{\Delta t, \pm}$ and the notation $(4.22 \mathrm{a}, \mathrm{b})$.

It follows immediately from the bound on the second term on the left-hand side of (4.27) that (4.29b) holds for some limit $\underset{\sim}{g} \in L^{2}\left(0, T ; \underset{\sim}{L^{2}}(\Omega \times D)\right)$, which we need to identify. However for any $\underset{\sim}{\underset{\sim}{q}} \in L^{2}\left(0, T ; \underset{\sim}{C_{0}^{\infty}}(\Omega \times D)\right)$, it follows from $(2.7),(2.18 \mathrm{a}, \mathrm{b})$ and the compact support of $\underset{\sim}{\eta}$ on $D$ that $\left[\nabla_{q} \cdot\left(M_{\underset{2}{\frac{1}{2}}}^{\underset{\sim}{\eta})}\right] / M^{\frac{1}{2}} \in L^{2}\left(0, T ; L^{2}(\Omega \times D)\right)\right.$ and hence the above convergence implies, on noting (4.29a), that

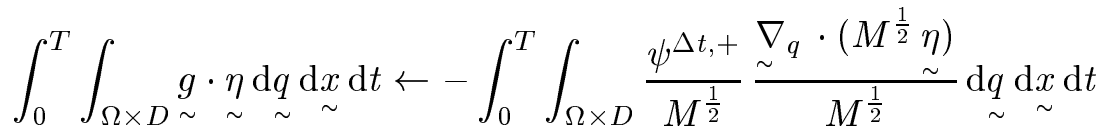

$$
\begin{aligned}
& \rightarrow-\int_{0}^{T} \int_{\Omega \times D} \frac{\psi}{M^{\frac{1}{2}}} \stackrel{\underset{\sim}{\nabla_{q} \cdot\left(M^{\frac{1}{2}} \underset{\sim}{\sim}\right)}}{M^{\frac{1}{2}}} \mathrm{~d} \underset{\sim}{\underset{\sim}{\mathrm{d}} x} \mathrm{~d} t \quad \text { as } \Delta t \rightarrow 0 .
\end{aligned}
$$

Hence the desired result (4.29b) follows from (4.32) on noting the denseness of $C_{0}^{\infty}(\Omega \times D)$ in $L^{2}(\Omega \times D)$. The desired result (4.29c) follows immediately from (4.29a), (2.2), (2.3) and (2.20).

The results $(4.30 \mathrm{a}-\mathrm{c})$ follow immediately from the bounds on the first four terms on the left-hand side of (4.28). The strong convergence result (4.30d) for ${\underset{\sim}{u}}^{\Delta t}$ follows immediately from (4.30a,c), (3.22) and (3.44), on noting that $\underset{\sim}{V} \subset \underset{\sim}{H}{ }_{0}^{1}(\Omega)$ is compactly embedded in $\underset{\sim}{L^{r}}(\Omega)$ for the stated values of $r$. We now prove (4.30d) for ${\underset{\sim}{\sim}}^{\Delta t, \pm}$. First we obtain from the bound on the second term on the left-hand side of (4.28) and (4.23) that

$$
\left\|u_{\sim}^{\Delta t}-u_{\sim}^{\Delta t, \pm}\right\|_{L^{2}\left(0, T, L^{2}(\Omega)\right)}^{2} \leq C \Delta t .
$$

Second, we note from Sobolev embedding that, for all $\eta \in L^{2}\left(0, T ; H^{1}(\Omega)\right)$,

$$
\|\eta\|_{L^{2}\left(0, T ; L^{r}(\Omega)\right)} \leq\|\eta\|_{L^{2}\left(0, T ; L^{2}(\Omega)\right)}^{\beta}\|\eta\|_{L^{2}\left(0, T ; L^{s}(\Omega)\right)}^{1-\beta} \leq C\|\eta\|_{L^{2}\left(0, T ; L^{2}(\Omega)\right)}^{\beta}\|\eta\|_{L^{2}\left(0, T ; H^{1}(\Omega)\right)}^{1-\beta}
$$

for all $r \in[2, s)$, with any $s \in(2, \infty)$ if $d=2$ or any $s \in(2,6]$ if $d=3$, and

$$
\beta=[2(s-r)] /[r(s-2)] \in(0,1] .
$$

Hence, combining (4.33), (4.34) and (4.30d) for ${\underset{\sim}{u}}^{\Delta t}$ yields (4.30d) for $\underset{\sim}{u}{ }^{\Delta t, \pm}$. Finally the desired result (4.30e) follows immediately from $(4.30 \mathrm{~d})$ and $(3.47 \mathrm{a})$. 
It follows from $(4.30 \mathrm{a}-\mathrm{d}),(4.29 \mathrm{c})$ and $(3.43)$ that we may pass to the limit, $\Delta t \rightarrow 0$, in $(4.24)$ to obtain that $\underset{\sim}{u} \in L^{\infty}\left(0, T ; \underset{\sim}{L^{2}}(\Omega)\right) \cap L^{2}(0, T ; \underset{\sim}{V}) \cap W^{1, \frac{4}{d}}\left(0, T ; V_{\sim}^{\prime}\right)$ and $\underset{\sim}{\tau}(\psi) \in L^{\infty}\left(0, T ; \underset{\sim}{L^{2}}(\Omega)\right)$ satisfy (3.51a). It also follows from (4.1a) that $\underset{\sim}{u}(\cdot, 0)=u_{0}(\cdot)$ in the required sense.

As we have no control of the time derivative or the $\underset{\sim}{x}$ derivatives of $\psi^{\Delta t}$, in order to pass to the $\Delta t \rightarrow 0$ limit in (4.25) these derivatives have to be transferred to the test functions.

On noting (4.10) and (4.26), we have for any fixed $\varphi \in C_{0}^{\infty}((-T, T) ; \mathcal{K})$ and for $\Delta t$ sufficiently small that

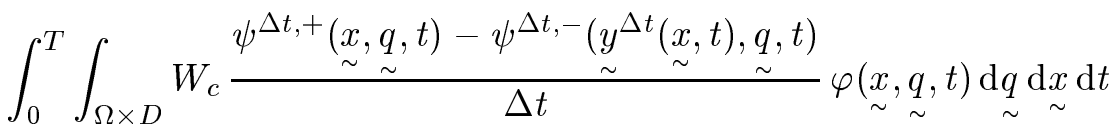

$$
\begin{aligned}
& \left.=-\int_{0}^{T} \int_{\Omega \times D} W_{c} \psi^{\Delta t,-} \underset{\sim}{x, q} \underset{\sim}{q}, t\right) \frac{\left.\varphi\left(\underset{\sim}{z^{\Delta t}}(\underset{\sim}{x}, t), \underset{\sim}{q}, t\right)-\varphi \underset{\sim}{\operatorname{s}}, \underset{\sim}{q}, t-\Delta t\right)}{\Delta t} \mathrm{~d} \underset{\sim}{q} \mathrm{~d} x \mathrm{~d} t \\
& -\int_{\Omega \times D} W_{c} \psi^{0} \underset{\sim}{\underset{\sim}{x}, \underset{\sim}{q})}\left(\frac{1}{\Delta t} \int_{0}^{t_{1}} \varphi(\underset{\sim}{x} \underset{\sim}{q}, t-\Delta t) \mathrm{d} t\right) \underset{\sim}{\mathrm{d} q} \underset{\sim}{\mathrm{d} x} .
\end{aligned}
$$

It follows from $(4.26),(4.11 \mathrm{~b})$ and $(4.28)$, for all $\varphi \in C_{0}^{\infty}((-T, T) ; \mathcal{K})$ and for all $\left.\underset{\sim}{\underset{\sim}{q}} \underset{\sim}{q}, t\right) \in$ $\Omega \times D \times(0, T)$ that

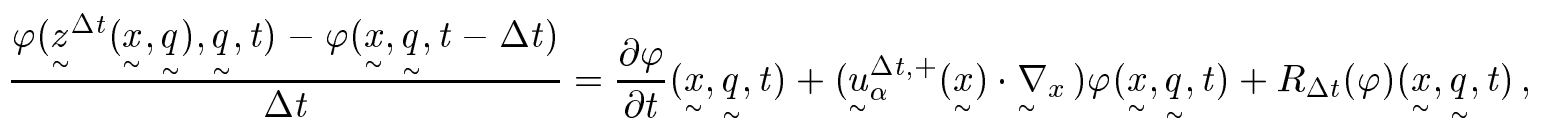

where

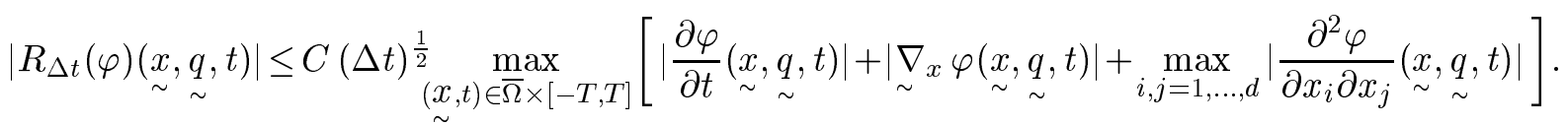

Hence, on combining (4.25), (4.35) and (4.36), we have for any fixed $\varphi \in C_{0}^{\infty}((-T, T) ; \mathcal{K})$ and for $\Delta t$ sufficiently small that

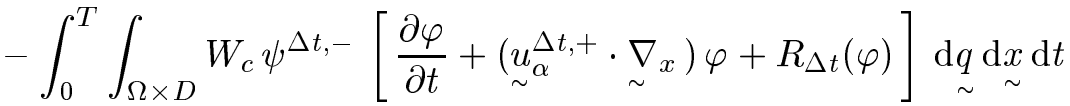

$$
\begin{aligned}
& \left.-\int_{\Omega \times D} W_{c} \psi^{0} \underset{\sim}{x, \underset{\sim}{q})}\left(\frac{1}{\Delta t} \int_{0}^{t_{1}} \varphi \underset{\sim}{x} \underset{\sim}{q}, t-\Delta t\right) \mathrm{d} t\right) \mathrm{d} \underset{\sim}{q} \mathrm{~d} x
\end{aligned}
$$

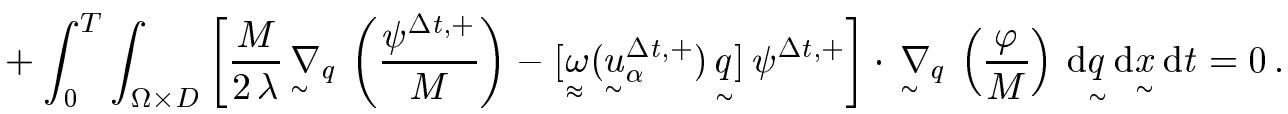

It follows from $(4.29 \mathrm{a}, \mathrm{b}),(4.30 \mathrm{e}),(3.47 \mathrm{a}),(4.36),(4.14 \mathrm{c})$ and $(4.1 \mathrm{~b})$ that we may pass to the limit $\Delta t \rightarrow 0$ in (4.37) to obtain that $\psi \in L^{2}(0, T ; K)$ with $M^{-\frac{1}{2}} \psi \in L^{\infty}\left(0, T ; L^{2}(\Omega \times D)\right)$ and ${\underset{\sim}{\alpha}}_{\alpha}:=\underset{\sim}{S} \underset{\sim}{u} \in L^{2}\left(0, T ; W^{1, \infty}(\Omega)\right)$ satisfy

$$
\begin{aligned}
& \left.-\int_{0}^{T} \int_{\Omega \times D} \frac{\psi}{M}\left[\frac{\partial \varphi}{\partial t}+\underset{\sim}{u_{\alpha}} \cdot \underset{\sim}{\nabla} x\right) \varphi\right] \underset{\sim}{\mathrm{d} q} \underset{\sim}{\mathrm{d}} \underset{\sim}{x} \mathrm{~d} t-\int_{\Omega \times D} \frac{\psi_{0}(\cdot, \cdot)}{M} \varphi(\cdot, \cdot, 0) \underset{\sim}{\mathrm{d} q} \underset{\sim}{\mathrm{d} x}
\end{aligned}
$$

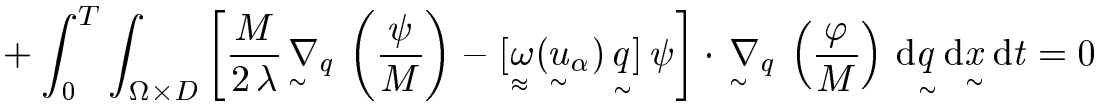

$$
\begin{aligned}
& \forall \varphi \in C_{0}^{\infty}((-T, T) ; \mathcal{K}) .
\end{aligned}
$$


Noting that, by Lemma $3.1, C_{0}^{\infty}((-T, T) ; \mathcal{K}$ ) is a dense subset of $\mathcal{X}$ (recall (3.50)), it follows that (4.38) remains true for all $\varphi \in \mathcal{X}$. Hence we have proved existence of a global weak solution to the weak formulation $(3.51 \mathrm{a}, \mathrm{b})$ of the smoothed corotational model.

Finally, we note that in the present corotational case one can derive an upper bound on $\psi^{n}$. To do so, we proceed inductively. Assuming that for some $L^{n-1} \in \mathbb{R}^{+}, \psi^{n-1} \leq L^{n-1} M$ a.e. in $\Omega \times D$, we then determine $L^{n} \in \mathbb{R}^{+}$in terms of $L^{n-1}$ such that $\psi^{n} \leq L^{n} M$ a.e. in $\Omega \times D$. Now, from Lemma 3.3, (4.13), (4.14a,b) and (4.15), we have, for any $L^{n} \in \mathbb{R}^{+}$, that $\left[\psi^{n}-L^{n} M\right]_{+} \in K_{q}$ and

$$
\begin{aligned}
& a_{c}^{n}\left(\left[\psi^{n}-L^{n} M\right]_{+},\left[\psi^{n}-L^{n} M\right]_{+}\right)=a_{c}^{n}\left(\psi^{n},\left[\psi^{n}-L^{n} M\right]_{+}\right)-L^{n} a_{c}^{n}\left(M,\left[\psi^{n}-L^{n} M\right]_{+}\right) \\
& =\ell_{c}^{n}\left(\left[\psi^{n}-L^{n} M\right]_{+}\right)-L^{n} a_{c}^{n}\left(M,\left[\psi^{n}-L^{n} M\right]_{+}\right)
\end{aligned}
$$

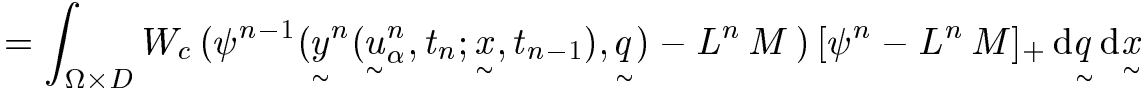

$$
\begin{aligned}
& \leq \int_{\Omega \times D}\left[W_{c}\left(L^{n-1}-L^{n}\right) M\right]\left[\psi^{n}-L^{n} M\right]_{+} \mathrm{d} \underset{\sim}{q} \mathrm{~d} x .
\end{aligned}
$$

On choosing $L^{n}=L^{n-1}$ yields that the right-hand side of (4.39) is zero and hence from the coercivity of $a_{c}^{n}(\cdot, \cdot)$ that $\left[\psi^{n}-L^{n} M\right]_{+} \equiv 0$. Thus, by induction, we have for $n=1 \rightarrow N$ that

$$
0 \leq \psi^{n} \leq L^{n} M=L^{0} M \quad \text { a.e. in } \Omega \times D, \quad \text { where } \quad L^{0}:=\sup _{\underset{\sim}{x, q} \underset{\sim}{q} \in \Omega \times D} \frac{\psi^{0}(\underset{\sim}{x}, q)}{M(\underset{\sim}{q})} .
$$

If $L^{0}$ is finite, then on recalling the notation (4.22a,b), (4.40) gives rise to a uniform $L^{\infty}(0, T$; $\left.L^{\infty}(\Omega \times D)\right)$ bound on $M^{-1} \psi^{\Delta(, \pm)}$. Moreover, it is then easily established that the limit $M^{-1} \psi \epsilon$ $L^{\infty}\left(0, T ; L^{\infty}(\Omega \times D)\right)$ with $\psi \geq 0$ a.e. on $\Omega \times D \times(0, T)$, and hence the norm $\|\cdot\|_{\mathcal{X}}$ can be relaxed to the weaker norm $\|\varphi\|_{L^{2}\left(0, T ; K_{q}\right)}+\left\|\frac{\partial \varphi}{\partial t}\right\|_{L^{1}\left(0 ; T ; L^{1}(\Omega \times D)\right)}+\left\|\nabla_{x} \varphi\right\|_{L^{1}\left(0, T ; L^{1}(\Omega \times D)\right)}$.

\subsection{Noncorotational models}

In order to mimic the formal energy estimate (3.41), we introduce a discrete-time procedure. Unlike the corotational case above, it does not appear possible to decouple the Navier-Stokes system from the probability density equation at each time level and still mimic (3.41). As stated previously, we need smoothing on the right-hand side of (3.52a) in order to cancel the smoothed drag term in $(3.52 b)$.

Let

$$
A^{0}(\underset{\sim}{v}):=\sup _{x \in \Omega}|\underset{\sim}{\nabla} \underset{\sim}{v} \underset{\sim}{v} \underset{\sim}{(x)}|^{2} \quad \underset{\sim}{\forall v} \in \underset{\sim}{V} \cap \underset{\sim}{W^{1, \infty}}(\Omega)
$$

Then, for $n=1 \rightarrow N$, given $\left\{{\underset{\sim}{u}}^{n-1}, A^{n-1}, \psi^{n-1}\right\} \in \underset{\sim}{V} \times \mathbb{R}^{+} \times K_{q}^{+}$, where $A^{n-1}=A^{n-1}\left({\underset{\sim}{\alpha}}_{\alpha}^{n-1}\right)$; 
find $\left\{\sim_{\sim}^{n}, A^{n}\left({\underset{\sim}{u}}_{\alpha}^{n}\right), \psi^{n}\right\} \in \underset{\sim}{V} \times \mathbb{R}^{+} \times K_{q}$ such that



$$
\begin{aligned}
& \left.=-\int_{\Omega} \underset{\sim}{\tau}\left(\psi^{n}\right): \underset{\approx}{\nabla_{x}} \underset{\sim}{\left(S_{\alpha} w\right.} \underset{\sim}{w}\right) \mathrm{d} x \quad \forall \underset{\sim}{w} \in \underset{\sim}{V}, \\
& \int_{\Omega \times D} \frac{\left.\psi^{n} \underset{\sim}{x} \underset{\sim}{q}\right)-\psi^{n-1}\left(\underset{\sim}{\left.y^{n}\left(\sim_{\alpha}^{n}, t_{n} ; \underset{\sim}{x}, t_{n-1}\right), \underset{\sim}{q}\right)}\right.}{\Delta t} \frac{\varphi}{M} \underset{\sim}{q} \underset{\sim}{\mathrm{d} x}
\end{aligned}
$$

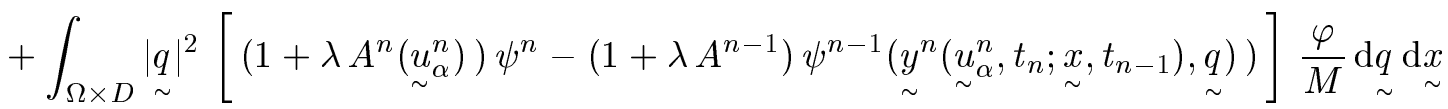

$$
\begin{aligned}
& +\frac{1}{2 \lambda} \int_{\Omega \times D} M \underset{\sim}{\nabla_{q}}\left(\frac{\psi^{n}}{M}\right) \cdot \underset{\sim}{\nabla_{q}}\left(\frac{\varphi}{M}\right) \underset{\sim}{\mathrm{d} q} \mathrm{~d} \underset{\sim}{x} \\
& =\int_{\Omega \times D} \psi^{n}\left[\left(\underset{\sim}{\nabla} \underset{\sim}{\underset{\sim}{u_{\alpha}}}\right) \underset{\sim}{q}\right] \cdot \underset{\sim}{\nabla_{q}}\left(\frac{\varphi}{M}\right) \underset{\sim}{\mathrm{d} q} \mathrm{~d} x \quad \forall \varphi \in K_{q} ;
\end{aligned}
$$

where $\underset{\sim}{u}:=\underset{\sim}{S}{ }_{\alpha} u^{n}$ and, for all $\underset{\sim}{v} \in \underset{\sim}{V} \cap \underset{\sim}{W^{1, \infty}}(\Omega)$,

$$
A^{n}(\underset{\sim}{v}):=\left.\sup _{x \in \Omega}\left|\nabla_{\sim} \underset{\sim}{v} \underset{\sim}{v} \underset{\sim}{x}\right|\right|^{2}
$$

Similarly to (4.3), it is convenient to rewrite (4.42a) as

$$
\left.b^{n}\left(\underset{\sim}{u^{n}}, \underset{\sim}{w}\right)=\int_{\Omega}\left[{\underset{\sim}{u}}^{n-1} \cdot \underset{\sim}{w}-\Delta t \underset{\approx}{\tau}\left(\psi^{n}\right): \underset{\approx}{\nabla} \underset{\sim}{\underset{\sim}{S}} \underset{\sim}{w}\right)\right] \underset{\sim}{\mathrm{d} x} \quad \forall \underset{\sim}{\forall} \in \underset{\sim}{V},
$$

where $b^{n}(\cdot, \cdot)$ is defined as in (4.4). It is also convenient to rewrite $(4.42 \mathrm{~b})$ as

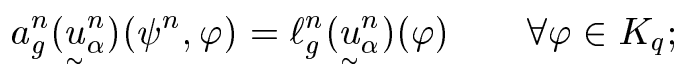

where, for all $\varphi_{1}, \varphi_{2}, \varphi \in K_{q}$ and $\underset{\sim}{v} \in \underset{\sim}{V} \cap W^{1, \infty}(\Omega)$,

$$
\begin{aligned}
& a_{g}^{n}(\underset{\sim}{v})\left(\varphi_{1}, \varphi_{2}\right):=\int_{\Omega \times D}\left(W_{g}^{n} \underset{\sim}{v}\right) \varphi_{1} \varphi_{2}+\Delta t\left[\frac{M}{2 \lambda} \underset{\sim}{\nabla_{q}}\left(\frac{\varphi_{1}}{M}\right)-\left[\left(\underset{\sim}{\nabla_{x}} \underset{\sim}{v} \underset{\sim}{q}\right] \varphi_{1}\right] \cdot \underset{\sim}{\nabla_{q}}\left(\frac{\varphi_{2}}{M}\right)\right) \underset{\sim}{\mathrm{d} q} \mathrm{~d} \underset{\sim}{\sim}, \\
& \left.\ell_{g}^{n} \underset{\sim}{v}(\varphi):=\int_{\Omega \times D} W_{g}^{n-1} \psi^{n-1} \underset{\sim}{\left(y^{n}\right.} \underset{\sim}{v}, t_{n} ; \underset{\sim}{x}, t_{n-1}\right), \underset{\sim}{q)} \underset{\sim}{\operatorname{d}} \underset{\sim}{\mathrm{d}} \underset{\sim}{\mathrm{d}} \underset{\sim}{1} \\
& W_{g}^{n}(\underset{\sim}{v}):=\frac{1+\Delta t|q|_{\sim}^{2}\left(1+\lambda A^{n}(\underset{\sim}{v})\right)}{M} \text { and } W_{g}^{n-1}=\frac{1+\Delta t|q|^{2}\left(1+\lambda A^{n-1}\right)}{M} .
\end{aligned}
$$

On noting that $\underset{\sim}{v} \in \underset{\sim}{V} \cap \underset{\sim}{W^{1, \infty}}(\Omega)$ and $(4.10)$, it follows that $a_{q}^{n}(\underset{\sim}{v})(\cdot, \cdot)$ is a continuous nonsymmetric bilinear functional on $K_{q} \times K_{q}$ and $\ell_{g}^{n}(\underset{\sim}{v})(\cdot)$ is a linear functional on $K_{q}$. Moreover, on applying a Young's inequality, we see that

$$
\left.a_{g}^{n} \underset{\sim}{v}\right)(\varphi, \varphi) \geq \int_{\Omega \times D}\left[W_{c}|\varphi|^{2}+\left.\frac{\Delta t M}{4 \lambda}||_{\sim} \nabla_{q}\left(\frac{\varphi}{M}\right)\right|^{2}\right] \underset{\sim}{\mathrm{d} q} \underset{\sim}{\mathrm{d} x} \quad \forall \varphi \in K_{q} ;
$$

that is, $a_{g}^{n}(\underset{\sim}{v})(\cdot, \cdot)$ is coercive on $K_{q} \times K_{q}$. 
In order to prove existence of a solution to (4.42a,b), we consider a fixed point argument. Given $\underset{\sim}{\widehat{u}} \in \underset{\sim}{L^{r}}(\Omega), r>d$, let $\left\{\psi^{\star}, u_{\sim}^{\star}\right\} \in K_{q} \times \underset{\sim}{V}$ be such that

$$
\begin{aligned}
a_{g}^{n}(\underset{\sim \alpha}{\widehat{u}})\left(\psi^{\star}, \varphi\right) & =\ell_{g}^{n}(\underset{\sim \alpha}{\widehat{u}})(\varphi) & \forall \varphi \in K_{q}, \\
b^{n}\left(\underset{\sim}{u^{\star}}, \underset{\sim}{w}\right) & =\int_{\Omega}\left[{\underset{\sim}{u}}^{n-1} \cdot \underset{\sim}{w}-\Delta t \underset{\sim}{\tau}\left(\psi^{\star}\right): \underset{\sim}{\nabla_{x}} \underset{\sim}{\left.S_{\alpha} w\right)} \underset{\sim}{\mathrm{d} x} \underset{\sim}{x}\right. & \forall \underset{\sim}{w} \in \underset{\sim}{V} ;
\end{aligned}
$$

where, on recalling (3.47a), $\widehat{\sim}_{\alpha}:={\underset{\sim}{\alpha}}_{\alpha} \widehat{\sim} \in \underset{\sim}{V} \cap W^{1, \infty}(\Omega)$. On noting (4.47), the Lax-Milgram theorem yields the existence of a unique solution to (4.48a). Similarly to (4.3), on noting (3.45b), there exists a unique solution to $(4.48 \mathrm{~b})$. Therefore the overall procedure $(4.48 \mathrm{a}, \mathrm{b})$ is well-posed.

Lemma 4.2. Let $\underset{\sim}{G}: \underset{\sim}{L^{r}}(\Omega) \rightarrow \underset{\sim}{V} \subset \underset{\sim}{L^{r}}(\Omega), r \in(d, 6)$, denote the nonlinear map that takes $\widehat{\sim} \underset{\sim}{t}$ to $\widetilde{u}^{\star}=G(\underset{\sim}{\widehat{u}})$ via the procedure $(4.48 a, b)$. Then $G$ has a fixed point. Hence there exists a solution $\left\{\sim_{\sim}^{n}, \widetilde{A}^{n}\left(\tilde{\sim}_{\alpha}^{n}\right), \psi^{n}\right\} \in \underset{\sim}{V} \times \mathbb{R}^{+} \times K_{q}$ to $(4 \cdot 42 a, b)$.

Proof. Clearly, a fixed point of $G$ yields a solution of $(4.42 \mathrm{a}, \mathrm{b})$. In order to show that $\underset{\sim}{G}$ has a fixed point, we apply Schauder's fixed point theorem; that is, we need to show that (i) $\underset{\sim}{G}: \underset{\sim}{\operatorname{L}}(\Omega) \rightarrow \underset{\sim}{L^{r}}(\Omega), r \in(d, 6)$, is continuous, (ii) compact, and (iii) there exists a $C_{\star} \in \mathbb{R}^{+}$such that

$$
\|\widehat{\sim}\|_{L^{r}(\Omega)} \leq C_{\star}
$$

for every $\underset{\sim}{\widehat{u}} \in \underset{\sim}{L^{r}}(\Omega)$ and $\beta \in(0,1]$ satisfying $\left.\underset{\sim}{\widehat{u}}=\beta G \underset{\sim}{(\widehat{u}}\right)$.

Let $\left\{\widehat{u}^{(i)}\right\}_{i \geq 0}$ be such that



We need to show that

$$
\widehat{\sim}^{(i)}:=\underset{\sim}{G}\left(\widehat{\sim}_{\sim}^{(i)}\right) \rightarrow \underset{\sim}{G}(\underset{\sim}{\widehat{u}}) \quad \text { strongly in } \underset{\sim}{L^{r}}(\Omega) \text { as } i \rightarrow \infty,
$$

in order to prove (i) above. We have from the definition of $G$, see $(4.48 \mathrm{a}, \mathrm{b})$, that, for all $i \geq 0$,

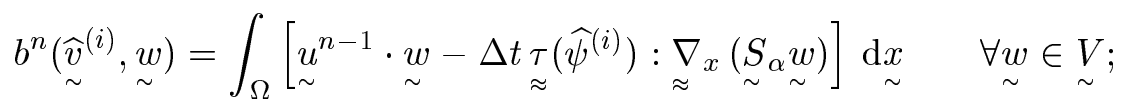

where $\widehat{\psi}^{(i)} \in K_{q}$ satisfies

$$
\left.a_{g}^{n} \underset{\sim \alpha}{\left(\widehat{u}^{(i)}\right.}\right)\left(\widehat{\psi}^{(i)}, \varphi\right)=\ell_{g}^{n}\left(\widehat{\sim}_{\sim \alpha}^{(i)}\right)(\varphi) \quad \forall \varphi \in K_{q},
$$

and from (3.47a) we have that

$$
\widehat{\sim}_{\sim \alpha}^{(i)}:=\underset{\sim}{S_{\alpha}} \underset{\sim}{\widehat{u}^{(i)}} \rightarrow \underset{\sim \alpha}{\widehat{u}}:=\underset{\sim}{S_{\alpha} \widehat{\sim}} \quad \text { strongly in } \underset{\sim}{W^{2, r}}(\Omega) \subset \underset{\sim}{W^{1, \infty}}(\Omega) \quad \text { as } i \rightarrow \infty .
$$

Choosing $\underset{\sim}{w} \equiv \underset{\sim}{v}(i)$ in (4.52a), and noting (3.25), (4.5), (2.2), (3.45b) and (3.27), yields, similarly to (4.6), that, for all $i \geq 0, \widehat{\sim}^{(i)} \in \underset{\sim}{V}$ satisfies

$$
\begin{aligned}
\int_{\Omega}\left[\left|\widehat{v}_{\sim}^{(i)}\right|^{2}+\left|\widehat{\sim}^{(i)}-u_{\sim}^{n-1}\right|^{2}-\left|u_{\sim}^{n-1}\right|^{2}\right] \mathrm{d} x & +\Delta t \nu \int_{\Omega} \mid \underset{\sim}{\mid} \underset{\sim}{\left.\widehat{v}_{\sim}^{(i)}\right|^{2}} \underset{\sim}{\mathrm{d} x} \\
& \leq C \Delta t \int_{\Omega \times D} \frac{\left|\widehat{\psi}^{(i)}\right|^{2}}{M} \mathrm{~d} \underset{\sim}{\mathrm{d}} \underset{\sim}{\mathrm{d} x} ;
\end{aligned}
$$


Choosing $\varphi \equiv \widehat{\psi}^{(i)}$ in (4.52b), and noting (4.47), (4.46b,c), (4.14c) and (4.10) yields, for all $i \geq 0$, that

$$
\begin{aligned}
\int_{\Omega \times D}\left[W_{c}\left|\widehat{\psi}^{(i)}\right|^{2}+\frac{\Delta t M}{2 \lambda}\left|\underset{\sim}{\nabla_{q}}\left(\frac{\widehat{\psi}^{(i)}}{M}\right)\right|^{2}\right] \underset{\sim}{\mathrm{d} \underset{\sim}{\mathrm{d}} \underset{\sim}{x}} \\
\leq\left(1+\lambda A^{n-1}\right)^{2} \int_{\Omega \times D} W_{c}\left|\psi^{n-1}\right|^{2} \underset{\sim}{\mathrm{d} q} \underset{\sim}{\mathrm{d}} \leq C .
\end{aligned}
$$

On combining (4.53) and (4.54), and noting an embedding result, see (3.21), and a Poincaré inequality, we have for all $i \geq 0$ that

$$
\left\|\widehat{\sim}^{(i)}\right\|_{L^{r}(\Omega)} \leq C \underset{\approx}{\|} \underset{\sim}{\widehat{v}^{(i)}} \|_{L^{2}(\Omega)} \leq C .
$$

Similarly to the proof of Lemma 4.1, it follows from (4.54) and (4.55), on noting the compactness of the embedding $\underset{\sim}{H}(\Omega) \hookrightarrow \underset{\sim}{L^{r}}(\Omega), r \in(d, 6)$, that there exists a subsequence $\left\{\widehat{\psi}^{\left(i_{k}\right)}, \widehat{\sim}^{\left(i_{k}\right)}\right\}_{i_{k} \geq 0}$ and functions $\widehat{\psi} \in K_{q}$ and $\underset{\sim}{\widehat{v}} \in \underset{\sim}{V}$ such that

$$
\begin{aligned}
& W_{c}^{\frac{1}{2}} \widehat{\psi}^{\left(i_{k}\right)} \rightarrow W_{c}^{\frac{1}{2}} \widehat{\psi} \quad \text { weakly in } L^{2}(\Omega \times D) \quad \text { as } i_{k} \rightarrow \infty \text {, } \\
& M^{\frac{1}{2}} \underset{\sim}{\nabla_{q}}\left(\frac{\widehat{\psi}^{\left(i_{k}\right)}}{M}\right) \rightarrow M^{\frac{1}{2}} \underset{\sim}{\nabla_{q}}\left(\frac{\widehat{\psi}}{M}\right) \quad \text { weakly in } \underset{\sim}{L^{2}}(\Omega \times D) \quad \text { as } i_{k} \rightarrow \infty \text {, } \\
& \underset{\approx}{\tau}\left(\widehat{\psi}^{\left(i_{k}\right)}\right) \rightarrow \underset{\approx}{\tau}(\widehat{\psi}) \quad \text { weakly in } \underset{\approx}{L^{2}}(\Omega \times D) \quad \text { as } i_{k} \rightarrow \infty \text {, } \\
& \stackrel{\sim}{\sim}^{\left(i_{k}\right)} \rightarrow \underset{\sim}{\widehat{v}} \quad \text { weakly in } \underset{\sim}{H^{1}}(\Omega) \quad \text { as } i_{k} \rightarrow \infty \text {, }
\end{aligned}
$$

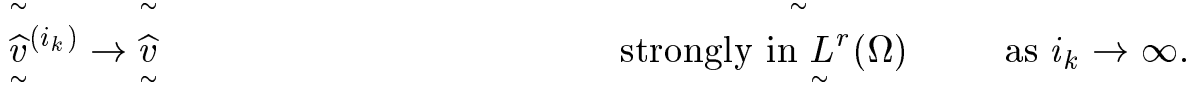

It follows from (4.52a), (4.4) and (4.56c,d), that $\underset{\sim}{\widehat{v}} \in \underset{\sim}{V}$ and $\widehat{\psi} \in K_{q}$ satisfy

$$
\left.b^{n}(\underset{\sim}{\widehat{v}}, \underset{\sim}{w})=\int_{\Omega}\left[u_{\sim}^{n-1} \cdot \underset{\sim}{w}-\Delta t \underset{\sim}{\tau}(\widehat{\psi}): \underset{\approx}{\nabla_{x}} \underset{\sim}{S_{\alpha} w} \underset{\sim}{w}\right)\right] \underset{\sim}{\mathrm{d} x} \quad \forall \underset{\sim}{w} \in \underset{\sim}{V} .
$$

For a fixed $\varphi \in K_{q}$, and any $\delta>0$, there exists, on recalling the density of $\mathcal{K}$ in $K_{q}$ (cf. the line below (3.49)), a $\varphi_{\delta} \in \mathcal{K}$ such that for all $\underset{\sim}{v} \in \underset{\sim}{V} \cap{\underset{\sim}{0}}^{0,1}(\bar{\Omega})$

$$
\left.\mid \ell_{g}^{n} \underset{\sim}{v}\right)\left(\varphi-\varphi_{\delta}\right) \mid \leq C\left\|\varphi-\varphi_{\delta}\right\|_{K_{q}} \leq \delta .
$$

We have from $(4.46 \mathrm{~b}),(4.9),(4.10)$ and $(4.12)$ that, for all ${\underset{\sim}{a}}_{a},{\underset{\sim}{b}}_{b} \in C^{0,1}(\Omega)$,

$$
\begin{aligned}
& \left.\mid \ell_{g}^{n} \underset{\sim}{\left(v_{a}\right.}\right)\left(\varphi_{\delta}\right)-\ell_{g}^{n}\left(v_{\sim}\right)\left(\varphi_{\delta}\right) \mid \\
& \left.\left.\left.=\mid \int_{\Omega \times D} W_{g}^{n-1} \psi^{n-1} \underset{\sim}{x} \underset{\sim}{q}\right)\left[\varphi_{\delta}\left(\underset{\sim}{y^{n}}\left(\underset{\sim}{v_{a}}, t_{n-1} ; \underset{\sim}{x}, t_{n}\right), \underset{\sim}{q}\right)-\varphi_{\delta} \underset{\sim}{\left(y^{n}\right.} \underset{\sim}{v_{b}}, t_{n-1} ; \underset{\sim}{x}, t_{n}\right), \underset{\sim}{q}\right)\right] \underset{\sim}{\mathrm{d} q} \mathrm{~d} x \mid \\
& \left.\leq C\left\|\underset{\sim}{\nabla_{x}} \varphi_{\delta}\right\|_{L^{\infty}(\Omega \times D)} \| \operatorname{\sim }_{\sim}^{n}\left(\underset{\sim}{v_{a}}, t_{n-1} ; \underset{\sim}{x}, t_{n}\right)-{\underset{\sim}{n}}_{\sim}^{n} \underset{\sim}{v_{b}}, t_{n-1} ; \underset{\sim}{x}, t_{n}\right) \|_{L^{\infty}(\Omega)} \\
& \leq C\left(\left\|{\underset{\sim}{v}}_{a}\right\|_{C^{0,1}(\bar{\Omega})},\left\|\underset{\sim}{v_{b}}\right\|_{C^{0,1}(\bar{\Omega})}\right)\left\|\underset{\sim}{\nabla} \varphi_{x} \varphi_{L^{\infty}(\Omega \times D)}\right\| v_{\sim} v_{a}-\underset{\sim}{v_{b}} \|_{L^{\infty}(\Omega)} \text {. }
\end{aligned}
$$

Combining (4.58) and (4.59) yields, on noting (4.52c), that

$$
\ell_{g}^{n}\left(\widehat{\sim}_{\sim \alpha}^{(i)}\right)(\varphi) \rightarrow \ell_{g}^{n}\left(\widehat{u}_{\sim \alpha}\right)(\varphi) \quad \text { as } i \rightarrow \infty, \quad \forall \varphi \in K_{q} .
$$


Hence it follows from (4.52b), (4.46a,c), (4.43), (4.56a,b), (4.52c) and (4.60) that $\widehat{\sim}_{\alpha}:=\underset{\sim}{\alpha} \widehat{\widehat{u}} \in$ $\underset{\sim}{V} \cap \underset{\sim}{W^{2, r}}(\Omega)$ and $\widehat{\psi} \in K_{q}$ satisfy

$$
a_{g}^{n}(\underset{\sim \alpha}{\widehat{u}})(\widehat{\psi}, \varphi)=\ell_{g}^{n}(\underset{\sim \alpha}{\widehat{u}})(\varphi) \quad \forall \varphi \in K_{q} .
$$

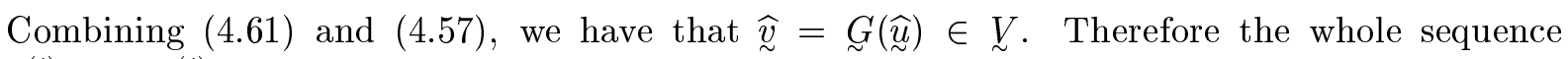



As the embedding $\underset{\sim}{V} \hookrightarrow \underset{\sim}{\operatorname{L}^{r}}(\Omega), r \in(d, 6)$, is compact; it follows that (ii) holds.

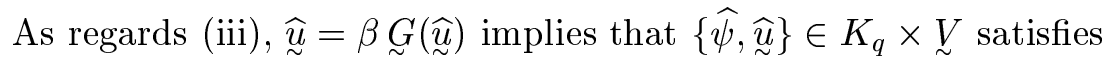

$$
\begin{aligned}
& a_{g}^{n}(\underset{\sim \alpha}{(\widehat{u}})(\widehat{\psi}, \varphi)=\ell_{g}^{n}\left(\underset{\sim \alpha}{\widehat{u}_{\alpha}}\right)(\varphi) \quad \forall \varphi \in K_{q}, \\
& \left.b^{n}(\underset{\sim}{\widehat{u}}, \underset{\sim}{w})=\beta \int_{\Omega}\left[u_{\sim}^{n-1} \cdot \underset{\sim}{w}-\Delta t \underset{\approx}{\tau}(\widehat{\psi}): \underset{\approx}{\nabla_{x}} \underset{\sim}{\left(S_{\alpha} w\right.}\right)\right] \mathrm{d} x \quad \underset{\sim}{\forall} \underset{\sim}{\underset{\sim}{V}} ;
\end{aligned}
$$

where $\widehat{\sim}_{\sim \alpha}:=S_{\alpha} \widehat{\sim}$. As $\psi^{n-1} \in K_{q}^{+}$and $\widehat{\psi} \in K_{q} \Longrightarrow[\widehat{\psi}]_{-} \in K_{q}$, recall Lemma 3.3, it follows from $(4.62 \mathrm{a})$ and $(4.46 \mathrm{a}, \mathrm{b})$ that

$$
a_{g}^{n}(\underset{\sim \alpha}{\widehat{u}})\left([\widehat{\psi}]_{-},[\widehat{\psi}]_{-}\right)=a_{g}^{n}(\underset{\sim \alpha}{(\widehat{u}})\left(\widehat{\psi},[\widehat{\psi}]_{-}\right)=\ell_{g}^{n}(\underset{\sim \alpha}{\widehat{u}})\left([\widehat{\psi}]_{-}\right) \leq 0 .
$$

Therefore (4.47) yields that $[\widehat{\psi}]_{-}=0$; that is, $\widehat{\psi} \in K_{q}^{+}$. On choosing $\underset{\sim}{w} \equiv \widehat{\sim}$ in $(4.62 \mathrm{~b})$, and noting (2.2) and that $\underset{\sim}{\underset{\sim}{\sim}} \in \underset{\sim}{V}$ yields, similarly to (4.6), that

$$
\begin{aligned}
\frac{1}{2} \int_{\Omega}\left[|\underset{\sim}{\mid \widehat{u}}|^{2}+\left.\left|\underset{\sim}{\widehat{u}}-\beta \underset{\sim}{\left.u^{n-1}\right|^{2}-\beta^{2}} \underset{\sim}{\left.\left|\sim^{n-1}\right|^{2}\right]} \underset{\sim}{\mathrm{d} x}+\Delta t \nu \int_{\Omega}\right| \nabla_{\approx} \underset{\sim}{\widehat{u}}\right|^{2}{\underset{\sim}{\mathrm{d}} x}\right. \\
=-\Delta t \beta k \mu \int_{\Omega} \underset{\sim}{C}(\widehat{\psi}): \underset{\sim}{\nabla_{x}} \underset{\sim \alpha}{\widehat{u}} \mathrm{~d} x .
\end{aligned}
$$

Choosing $\varphi=M$ in (4.62a) and noting (4.10) yields that

$$
\left.\int_{\Omega \times D}\left[1+\Delta t \underset{\sim}{|q|^{2}}\left(1+\lambda A^{n} \underset{\sim \alpha}{\widehat{u}}\right)\right)\right] \underset{\sim}{\widehat{\psi}} \underset{\sim}{q} \underset{\sim}{\mathrm{d} x}=\int_{\Omega \times D}\left[1+\Delta t \underset{\sim}{|q|^{2}}\left(1+\lambda A^{n-1}\right)\right] \psi^{n-1} \underset{\sim}{\mathrm{d} q} \underset{\sim}{\mathrm{d} x} .
$$

Choosing $\varphi=U M$ in (4.62a), and noting (2.8), (2.3), (4.10), Lemma 3.2, (2.7) and (2.17) yields, 
similarly to (3.40), that

$$
\begin{aligned}
& \int_{\Omega \times D}\left(\widehat{\psi}-\psi^{n-1}\right) U \underset{\sim}{\mathrm{d} q} \underset{\sim}{\mathrm{d} x}-\Delta t \int_{\Omega} \underset{\sim}{\approx}(\widehat{\psi}): \underset{\approx}{\nabla_{x}} \underset{\sim \alpha}{\widehat{u}} \mathrm{~d} \underset{\sim}{x} \\
& +\Delta t \int_{\Omega \times D}|q|^{2}\left[\left(1+\lambda A^{n}(\underset{\sim \alpha}{\widehat{u}})\right) \widehat{\psi}-\left(1+\lambda A^{n-1}\right) \psi^{n-1}\right] U \underset{\sim}{U \mathrm{~d} q} \mathrm{~d} x \\
& =-\frac{\Delta t}{2 \lambda} \int_{\Omega \times D} M \underset{\sim}{\underset{\sim}{\nabla}}\left(\frac{\widehat{\psi}}{M}\right) \cdot U_{\sim}^{\prime} \underset{\sim}{\mathrm{d} q} \underset{\sim}{\mathrm{d} x} \\
& =\frac{\Delta t}{2 \lambda} \int_{\Omega \times D}\left[\left(U^{\prime \prime}-\left(U^{\prime}\right)^{2}\right) \underset{\sim}{|q|^{2}}+d U^{\prime}\right] \underset{\sim}{\widehat{\psi}} \underset{\sim}{\mathrm{d} q} \underset{\sim}{\mathrm{d} x}
\end{aligned}
$$

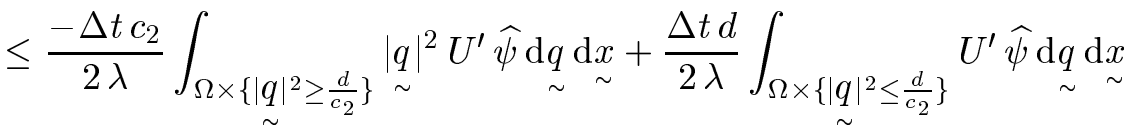

$$
\begin{aligned}
& \leq \frac{-\Delta t c_{2}}{2 \lambda} \int_{\Omega \times\left\{|q|^{2} \geq \frac{d}{c_{2}}\right\}}|q|^{2} U^{\prime} \widehat{\psi} \underset{\sim}{\sim} \mathrm{d} x \\
& \left.+C \Delta t \int_{\Omega \times D}\left[1+\Delta t \underset{\sim}{|q|^{2}}\left(1+\lambda A^{n} \underset{\sim \alpha}{\widehat{u}}\right)\right)\right] \underset{\sim}{\widehat{\psi}} \mathrm{d} q \underset{\sim}{\mathrm{d} x} .
\end{aligned}
$$

Combining (4.64) and (4.66) multiplied by $\beta k \mu$, and noting (4.65) yields that

$$
\begin{aligned}
& \frac{1}{2} \int_{\Omega}\left[|\underset{\sim}{\mid \widehat{u}}|^{2}+\left.\left|\underset{\sim}{\widehat{u}}-\beta \underset{\sim}{\left.\left.u^{n-1}\right|^{2}\right]} \mathrm{d} \underset{\sim}{x}+\Delta t \nu \int_{\Omega}\right| \nabla_{\approx} x \underset{\sim}{\widehat{u}}\right|^{2} \mathrm{~d} x\right. \\
& +\beta k \mu \int_{\Omega \times D}\left[1+\Delta t \underset{\sim}{|q|^{2}}\left(1+\lambda A_{\sim}^{n}(\underset{\sim \alpha}{\widehat{u}})\right)\right] U \underset{\sim}{\widehat{\psi}} \underset{\sim}{\mathrm{d} q} \mathrm{~d} x \\
& +\frac{\Delta t \beta k \mu c_{2}}{2 \lambda} \int_{\Omega \times\left\{|q|^{2} \geq \frac{d}{c_{2}}\right\}} \underset{\sim}{|q|^{2}} U^{\prime} \widehat{\psi} \underset{\sim}{\mathrm{d} q} \underset{\sim}{\mathrm{d} x} \\
& \leq \frac{1}{2} \beta^{2} \int_{\Omega} \mid \underset{\sim}{\left|u^{n-1}\right|^{2}} \underset{\sim}{\mathrm{d} x}+\beta k \mu \int_{\Omega \times D}\left[1+\left.\underset{\sim}{\Delta t} \underset{\sim}{\mid q}\right|^{2}\left(1+\lambda A^{n-1}\right)\right] U \psi^{n-1} \underset{\sim}{\mathrm{d} q} \underset{\sim}{\mathrm{d} x} \\
& +C \beta k \mu \Delta t \int_{\Omega \times D}\left[1+\Delta t \underset{\sim}{|q|^{2}}\left(1+\lambda A^{n-1}\right)\right] \psi^{n-1} \underset{\sim}{\mathrm{d}} \underset{\sim}{\mathrm{d} x} .
\end{aligned}
$$

As $\widehat{\psi} \in K_{q}^{+}$, then (4.67) gives rise to the desired bound (4.49) with $C$ dependent on $\Delta t$, on

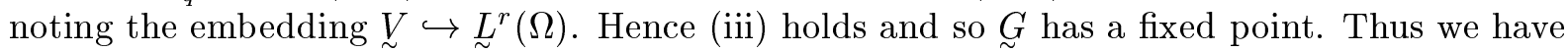
proved existence of a solution to $(4.42 \mathrm{a}, \mathrm{b})$.

Repeating the arguments (4.63)-(4.67) for the solution $\left\{u_{\sim}^{n}, \psi^{n}\right\}$ of $(4.42 \mathrm{a}, \mathrm{b})$ yields that $\psi^{n} \in K_{q}^{+}$and (4.67) holds with $\beta=1$ and $\{\underset{\sim}{\widehat{u}}, \widehat{\psi}\}$ replaced by $\left\{{\underset{u}{u}}^{n}, \psi^{n}\right\}$. Summing this from $n=1 \rightarrow m, m=1 \rightarrow N$, and noting by induction on (4.65) that

$$
\int_{\Omega \times D}\left[1+\Delta t \underset{\sim}{|q|^{2}}\left(1+\lambda A^{n-1}\right)\right] \psi^{n-1} \underset{\sim}{\mathrm{d} q} \underset{\sim}{\mathrm{d} x}=\int_{\Omega \times D}\left[1+\Delta t \underset{\sim}{|q|^{2}}\left(1+\lambda A^{0}\right)\right] \psi^{0} \mathrm{~d} \underset{\sim}{\mathrm{d} x} \underset{\sim}{\sim}
$$


and (4.20) yields the analogue of (3.42)

$$
\begin{aligned}
& \frac{1}{2}\left[\max _{n=1 \rightarrow N}\left[\int_{\Omega}\left|u_{\sim}^{n}\right|^{2} \mathrm{~d} \underset{\sim}{x}\right]+\sum_{n=1}^{N} \int_{\Omega} \mid u_{\sim}^{n}-\underset{\sim}{\left.u^{n-1}\right|^{2} \mathrm{~d} x} \underset{\sim}{\sim}\right]+\nu \sum_{n=1}^{N} \Delta t \int_{\Omega}\left|\underset{\sim}{\mid} \underset{\sim}{u_{\sim}^{n}}\right|^{2} \mathrm{~d} x \\
& +k \mu \max _{n=1 \rightarrow N}\left[\int_{\Omega \times D} U \psi^{n} \mathrm{~d} \underset{\sim}{\mathrm{d}} \underset{\sim}{\mathrm{d} x}\right]+\frac{k \mu c_{2}}{2 \lambda} \sum_{n=1}^{N} \Delta t \int_{\Omega \times\left\{|q|^{2} \geq \frac{d}{c_{2}}\right\}} \underset{\sim}{|q|^{2}} U^{\prime} \psi^{n} \underset{\sim}{\mathrm{d} q} \mathrm{~d} \underset{\sim}{\mathrm{d}} \\
& +\Delta t \max _{n=1 \rightarrow N}\left[\int_{\Omega \times D} \mid \underset{\sim}{|q|^{2}}\left(1+\lambda A^{n} \underset{\sim}{\left.\left.u_{\alpha}^{n}\right)\right) U} \psi^{n} \underset{\sim}{\mathrm{d} q} \underset{\sim}{\mathrm{d} x}\right]\right. \\
& \leq \frac{1}{2} \int_{\Omega}\left|{\underset{\sim}{0}}^{0}\right|^{2} \mathrm{~d} \underset{\sim}{x}+C(T) \int_{\Omega \times D}\left[1+\Delta t \underset{\sim}{|q|^{2}}\left(1+\lambda A^{0}\right)\right](1+U) \psi^{0} \mathrm{~d} \underset{\sim}{q} \mathrm{~d} x \\
& \leq C \text {. }
\end{aligned}
$$

The bounds on $\psi^{n}$ in (4.69) do not suffice in order to pass to the limit $\Delta t \rightarrow 0$ in the summation over $n$ of (4.42b). One needs to establish additional bounds on $\psi^{n}$. We confine ourselves to the physically more realistic case of FENE-type models.

It follows from (4.43), (3.47b), (3.21), (4.69) and (4.20) that, for any $\beta \in\left[1, \frac{1}{\gamma}\right]$,

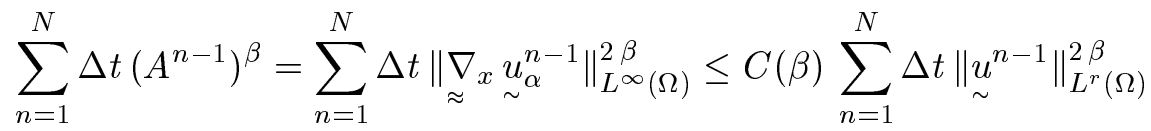

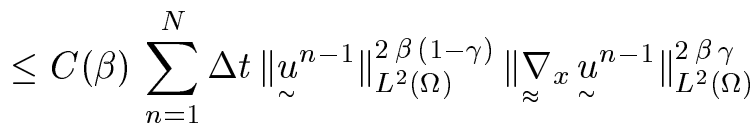

$$
\begin{aligned}
& \leq C(\beta)\left(\sum_{n=1}^{N} \Delta t\left\|\underset{\approx}{\nabla_{x}}{\underset{\sim}{u}}^{n-1}\right\|_{L^{2}(\Omega)}^{2}\right)^{\beta \gamma} \leq C_{1}(\beta),
\end{aligned}
$$

where $r \in(d, 6]$ and $\gamma \in[d(r-2) /(2 r), 1]$. Choosing $\varphi=\psi^{n}$ in (4.45) and noting (4.46c), (4.14c) and (4.47) yields that

$$
\begin{aligned}
& \left.\int_{\Omega \times D} W_{c} \psi^{n}\left(\psi^{n}-\psi^{n-1} \underset{\sim}{\left(y^{n}\right.} \underset{\sim}{u_{\alpha}^{n}}, t_{n} ; \underset{\sim}{x}, t_{n-1}\right), \underset{\sim}{q)}\right) \underset{\sim}{\mathrm{d}} \underset{\sim}{\mathrm{d}} \underset{\sim}{x}+\frac{\Delta t}{4 \lambda} \int_{\Omega \times D} M\left|\underset{\sim}{\nabla_{q}}\left(\frac{\psi^{n}}{M}\right)\right|^{2} \underset{\sim}{\mathrm{d} q} \mathrm{~d} \underset{\sim}{\sim}
\end{aligned}
$$

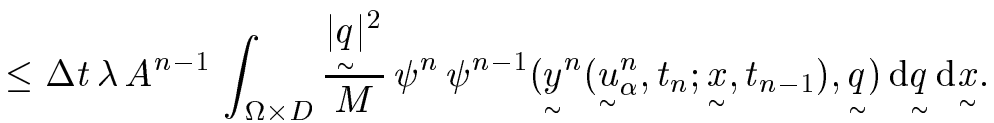

Applying the identity (4.5) and a Young's inequality to (4.71), and noting (4.10), that $D$ is bounded and (4.70) with $C_{1} \equiv C_{1}(1)$, yields

$$
\begin{aligned}
& \left.\left.\left(1-\frac{1}{2} C_{1}^{-1} \Delta t A^{n-1}\right) \int_{\Omega \times D} W_{c}\left|\psi^{n}\right|^{2} \underset{\sim}{\mathrm{d} q} \underset{\sim}{\mathrm{d}} \underset{\sim}{x}+\int_{\Omega \times D} W_{c} \mid \psi^{n}-\psi^{n-1} \underset{\sim}{\left(y^{n}\right.} \underset{\sim}{u_{\alpha}^{n}}, t_{n} ; \underset{\sim}{x}, t_{n-1}\right), \underset{\sim}{q}\right)\left.\right|^{2} \mathrm{~d} q \underset{\sim}{\mathrm{d} x} \\
& +\frac{\Delta t}{2 \lambda} \int_{\Omega \times D} M\left|\underset{\sim}{\nabla_{q}}\left(\frac{\psi^{n}}{M}\right)\right|^{2} \underset{\sim}{\mathrm{d} q} \underset{\sim}{\mathrm{d}} \underset{\sim}{x} \leq\left(1+C_{2} \Delta t A^{n-1}\right) \int_{\Omega \times D} W_{c}\left|\psi^{n-1}\right|^{2} \underset{\sim}{\mathrm{d} q} \underset{\sim}{\mathrm{d} x} .
\end{aligned}
$$

It follows from (4.72) that

$$
\begin{aligned}
\int_{\Omega \times D} W_{c}\left|\psi^{n}\right|^{2} \mathrm{~d} \underset{\sim}{\mathrm{d} x} & \leq \frac{1+C_{2} \Delta t A^{n-1}}{1-\frac{1}{2} C_{1}^{-1} \Delta t A^{n-1}} \int_{\Omega \times D} W_{c}\left|\psi^{n-1}\right|^{2} \underset{\sim}{\mathrm{d} q} \mathrm{~d} x \\
& \leq \mathrm{e}^{C \Delta t A^{n-1}} \int_{\Omega \times D} W_{c}\left|\psi^{n-1}\right|^{2} \mathrm{\sim} \underset{\sim}{\mathrm{d} x} .
\end{aligned}
$$


Hence combining (4.73) and (4.70) with $\beta=1$, summing (4.72) from $n=1 \rightarrow N$ and noting (3.27) yields the bounds (4.18a) for the general noncorotational FENE model; in particular:

$$
\begin{aligned}
& \max _{n=1 \rightarrow N}\left[\int_{\Omega \times D} W_{c}\left|\psi^{n}\right|^{2} \underset{\sim}{\mathrm{d} q} \mathrm{~d} \underset{\sim}{x}\right]+\frac{1}{\lambda} \sum_{n=1}^{N} \Delta t \int_{\Omega \times D} M\left|\underset{\sim}{\nabla_{q}}\left(\frac{\psi^{n}}{M}\right)\right|^{2} \underset{\sim}{\mathrm{d} q} \underset{\sim}{\mathrm{d} x} \\
& \left.\left.\left.\left.+\sum_{n=1}^{N} \int_{\Omega \times D} W_{c} \mid \psi^{n} \underset{\sim}{x} \underset{\sim}{q}\right)-\psi^{n-1} \underset{\sim}{\left(y^{n}\right.} \underset{\sim}{u_{\alpha}^{n}}, t_{n}, \underset{\sim}{x} ; t_{n-1}\right)\right), \underset{\sim}{q}\right)\left.\right|^{2} \underset{\sim}{\mathrm{d} q} \mathrm{~d} \underset{\sim}{x} \\
& +\max _{n=0 \rightarrow N}\left[\int_{\Omega}\left|C\left(\psi^{n}\right)\right|^{2} \mathrm{~d} x\right] \leq C
\end{aligned}
$$

Finally on choosing $\underset{\sim}{w} \equiv \underset{\sim}{S}\left(\frac{u^{n}-u^{n-1}}{\Delta t}\right) \in \underset{\sim}{V}$ in (4.42a) yields, on noting (3.45b), a direct analogue of (4.7) and (4.19):

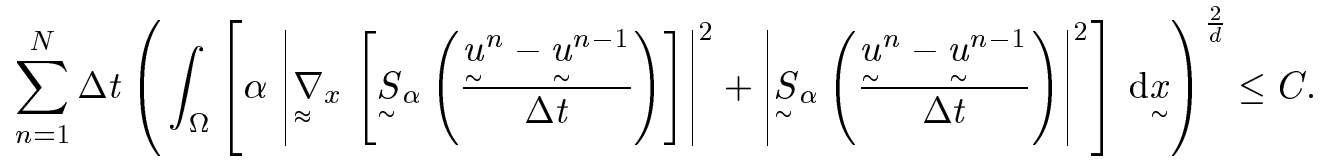

We have now established all of the analogues of the bounds (4.18a,b) and (4.19) in the corotational case for the general noncorotational FENE-type potentials, see (4.69), (4.74) and (4.75) above. The remainder of the convergence proof follows exactly the same arguments as in the corotational case. The only differences are: (i) the presence of $\underset{\sim}{S_{\alpha}}$ on the right-hand side of (4.42a); and (ii) the term involving $A^{n}(\underset{\sim}{u})$ in $a_{g}^{n}(\underset{\sim}{u})(\cdot, \cdot)$ and the term involving $A^{n-1}$ in $\ell_{g}^{n}(\cdot)$. Obviously (i) causes no difficulties whatsoever. Hence we comment only on (ii).

Therefore to prove existence of a solution to $(3.52 \mathrm{a}, \mathrm{b})$, we need only to show, on noting the notation (4.22a,b), that, for all $\varphi \in C_{0}^{\infty}((-T, T) ; \mathcal{K})$,

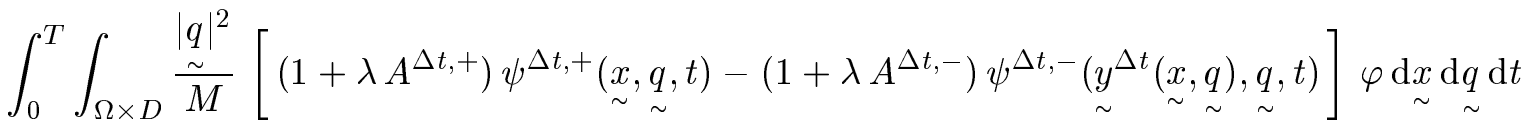

$$
\begin{aligned}
& \rightarrow 0 \quad \text { as } \Delta t \rightarrow 0 \text {, }
\end{aligned}
$$

where $A^{\Delta t,+}$ and $A^{\Delta t,-}$ are defined analogously to $\psi^{\Delta t,+},{\underset{\sim}{u}}^{\Delta t,+}$ and $\psi^{\Delta t,--},{\underset{\sim}{u}}^{\Delta t,--}$, respectively.

Now, similarly to (4.35), we have from (4.10), (4.26), (4.36), (4.74) and (4.70) for any $\varphi \in C_{0}^{\infty}((-T, T) ; \mathcal{K})$ that, for $\Delta t$ sufficiently small,

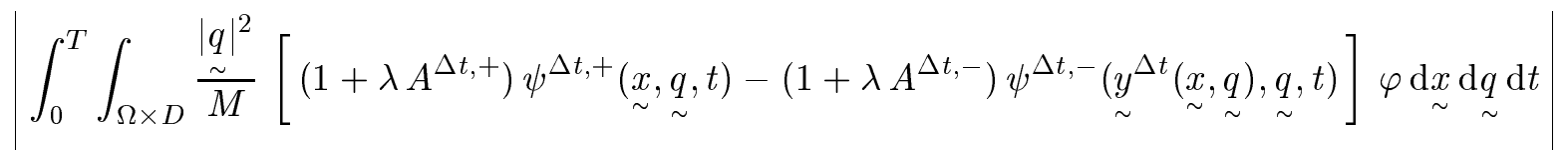

$$
\begin{aligned}
& \left.=\mid-\Delta t \int_{0}^{T} \int_{\Omega \times D} \frac{|q|^{2}}{\stackrel{\sim}{M}}\left(1+\lambda A^{\Delta t,-}\right) \psi^{\Delta t,-}\left[\frac{\partial \varphi}{\partial t}+\underset{\sim}{u_{\alpha}^{\Delta t,+}} \cdot \underset{\sim}{\nabla_{x}}\right) \varphi+R_{\Delta t}(\varphi)\right] \underset{\sim}{\mathrm{d} q} \underset{\sim}{\mathrm{d}} \underset{\sim}{x} \mathrm{~d} t \\
& \left.\left.-\Delta t \int_{\Omega \times D} \frac{|q|^{2}}{\stackrel{\sim}{M}}\left(1+\lambda A^{0}\right) \psi^{0} \underset{\sim}{x} \underset{\sim}{q}\right)\left(\frac{1}{\Delta t} \int_{0}^{t_{1}} \varphi \underset{\sim}{x} \underset{\sim}{q}, t-\Delta t\right) \mathrm{d} t\right) \underset{\sim}{\mathrm{d} q} \mathrm{~d} \underset{\sim}{x} \mid \\
& \leq C(\varphi) \Delta t \int_{0}^{T}\left(1+\lambda A^{\Delta t,-}\right) \mathrm{d} t \leq C(\varphi) \Delta t .
\end{aligned}
$$


Hence the desired result (4.76) holds. Therefore we have proved global existence of a solution to the weak formulation $(3.52 \mathrm{a}, \mathrm{b})$ of the smoothed noncorotational model for FENE-type potentials.

REMARK 4.1. The argument presented above for noncorotational FENE-type models breaks down for noncorotational Hookean models, since in the transition from bound (4.71) to (4.72) we exploit the fact that $D$ is bounded. The difficulty could be overcome if one could obtain a maximum principle on $\psi^{n}$ along the lines of (4.39). Unfortunately, in the case of $D=\mathbb{R}^{d}$ this does not appear to be readily achievable. Having said this, our main focus of interest in the present article have been FENE-type microscopic-macroscopic models for diluted polymers where $D$ is a bounded open ball in $\mathbb{R}^{d}$ : for, the fact that in Hookean-type models the domain $D$ is equal to the whole of $\mathbb{R}^{d}$ stems from the physically unrealistic modelling assumption that the length $|q|$ of the elongation-vector $\underset{\sim}{q} \in D$ of a polymer chain may be arbitrarily large.

REMARK 4.2. It is plausible that the existence of global weak solutions to the original model problem $(P)$ could be established, without smoothing of the model, by combining the ideas developed here with the DiPerna-Lions theory of renormalised solutions to linear first-order hyperbolic problems with coefficients in Sobolev spaces [5]; see also the more recent work of Ambrosio [1] on the subject of first-order linear hyperbolic PDEs with non-smooth coefficients. This line of investigation will, however, require a notion of weak solution different from the one considered here, and will be the subject of future research.

Acknowledgement: Part of this work was carried out while the authors participated in the Research Programme Computational Challenges in Partial Differential Equations at the Isaac Newton Institute, Cambridge, UK, between January and July 2003.

\section{References}

[1] Ambrosio, L., Transport equation and Cauchy problem for $B V$ vector fields. Inventiones Mathematicae (Accepted for publication), 2004.

[2] Besov, O.V. \& Kufner, A., On the density of smooth functions in weighted spaces. (In Russian). Czechoslovak Math. J. 18(93), (1968) 178-188.

[3] Bird, R., Curtiss, C., Armstrong, R., \& Hassager, O., Dynamics of Polymeric Liquids, Vol 2., Kinetic Theory. John Wiley \& Sons, New York, 1987.

[4] Degond, D., Lemou, M., \& Picasso, M., Viscoelastic fluid models derived from kinetic equations for polymers. SIAM J. Appl. Math. 62, (2002) 1501-1519.

[5] DiPerna R.J. \& Lions, P.-L., Ordinary differential equations, transport theory and Sobolev spaces. Inventiones Mathematicae 98, (1989) 511-547.

[6] Doi, M. \& Edwards, S.F., The Theory of Polymer Dynamics. Oxford University Press, Oxford, 1988.

[7] El-Kareh, A.W. \& Leal, L.G., Existence of solutions for all Deborah numbers for a non-Newtonian model modified to include diffusion. J. Non-Newtonian Fluid Mech. 33, (1989) 257-287. 
[8] Foias, C., Holm, D.D., \& Titi, E.S., The Navier-Stokes- $\alpha$ model of fluid turbulence. Physica D 152-153, (2001) 505-519.

[9] Girault, V. \& Raviart, P.A., Finite Element Methods for Navier-Stokes Equations. Springer Ser. Comp. Math. 5, Springer-Verlag, Berlin, 1986.

[10] Jourdain, B., Lelièvre, T., \& Le Bris, C., Numerical analysis of micro-macro simulations of polymeric fluid flows: a simple case. $M^{3} A S$ 12, (2002) 1205-1243.

[11] Jourdain, B., Lelièvre, T., \& Le Bris, C., Existence of solution for a micro-macro model of polymeric fluid: the FENE model. J. Funct. Anal. 209, (2004) 162-193.

[12] KellogG, R.B. \& Osborn, J., A regularity result for the Stokes problem in a convex polygon. J. Funct. Anal. 21, (1976) 397-431.

[13] Kozlov, V.A., Mazya, V.G., \& Schwab, C., On singularities of solutions to the Dirichlet problem of hydrodynamics near the vertex of a cone. Journal für die Reine und Angewandte Mathematik 456, (1994) 65-97.

[14] Kufner, A., Weighted Sobolev Spaces. Teubner-Texte zur Mathematik, Leipzig, 1980.

[15] Lions, P.L. \& Masmoudi, M., Global solutions for some Oldroyd models of nonNewtonian flows. Chin. Ann. of Math. B 21, (2000) 131-146.

[16] Lozinski, A., Chauvière, C., Fang, J., \& Owens, R.G., Fokker-Planck simulations of fast flows of melts and concentrated polymer solutions in complex geometries. J. Rheol. $\mathbf{4 7}(2),(2003), 535-561$.

[17] Öтtinger, H.-C., Stochastic Processes in Polymeric Fluids. Springer-Verlag, Berlin, 1996.

[18] Renardy, M., An existence theorem for model equations resulting from kinetic theories of polymer solutions. SIAM J. Math. Anal. 22, (1991) 313-327.

[19] Simon, J., Compact sets in the space $L^{p}(0, T ; B)$. Ann. Math. Pura. Appl. 146, (1987) $65-96$.

[20] Suen, J.K.C., Joo, Y.L., \& Armstrong, R.C., Molecular orientation effects in viscoelasticity. Annu. Rev. Fluid Mech. 34, (2002) 417-444.

[21] Temam, R., Navier-Stokes Equations. Theory and Numerical Analysis. With an appendix by F. Thomasset. Third edition. Studies in Mathematics and its Applications, 2. NorthHolland Publishing Co., Amsterdam, 1984.

[22] Triebel, H., Interpolation Theory, Function Spaces, Differential Operators. 2nd Ed., Joh. Ambrosius Barth Publ., Heidelberg, Leipzig, 1995. 